\title{
LOCAL BENDING CONTAINMENT IN SANDWICH PANELS WITH SUBSURFACE CORE DAMAGE
}

A Thesis by

Ritesh Ghimire

Bachelor of Aeronautical Engineering, Madras University, 2004

Submitted to the Department of Aerospace Engineering and the faculty of graduate school of

Wichita State University

in partial fulfillment of

the requirements for the degree of

Master of Science

May 2008 
(C) Copyright 2008 by Ritesh Ghimire

All Rights Reserved 


\section{LOCAL BENDING CONTAINMENT IN SANDWICH PANELS WITH SUBSURFACE CORE DAMAGE}

The following faculty members have examined the final copy of this thesis for form and content, and recommend that it be accepted in partial fulfillment of the requirements for the degree of Master of Science, with a major in Aerospace Engineering.

Keshavanarayana Suresh Raju, Committee Chair

Bob Minaie, Committee Member

Charles Yang, Committee Member 


\section{DEDICATION}

To my parents and friends 


\section{ACKNOWLEDGEMENTS}

I wish to express my sincere gratitude to my advisor Dr. Suresh K. Raju for suggesting this research. His valuable suggestions, guidance and patient association throughout the course of the research program had encouraged me a lot. My special thanks to Dr. Charles Yang and Dr. Bob Minaie for being in thesis committee.

I would like to make a special mention of my colleagues Juan Acosta Felipe, Stacy Ury, Satish Kumar, Anand Despande, Annapurna Kolachalama, Govind Pillai, Jackson Anyasi, Manoj Varma Thotakuri, Floyd Caiado, Sandeep Shetty and other engineers from National Institute for Aviation Research for their kind support in carrying out the research and cooperating with varieties of applications.

My thesis will remain incomplete without acknowledging the moral support of my parents, family members, lecturers and friends as the knowledge I have acquired from them has been of great value.

Finally I thank all those who directly or indirectly contributed to this work. 


\begin{abstract}
The compressive residual strength and behavior of impact damaged sandwich panels are governed by the amount of non-visible core damage underneath the facesheets. The failure modes under in-plane compressive loading are either due to crack precipitation from the damage zone or unstable dimple propagation across the width. In both cases, the local bending of facesheet (dimple) triggers the final failure sequence. Thus, containment of this local bending by reinforcing the core cell(s) in the damage region will mitigate the failure initiation and thus increase residual properties. A repair technique involving the containment of local bending of sandwich facesheet by strategically filling honeycomb core cells has been explored to improve the damage tolerance of impact damaged sandwich panels. The experimental results indicate that filling honeycomb cells at the center of the damage region in addition to those at the edge of the damage region produces the maximum benefit. The test data and final failure mode of the repaired specimen under in-plane compression loading indicated that the present repair technique can help recover the undamaged strength of the sandwich panels.
\end{abstract}




\section{TABLE OF CONTENTS}

Chapter

Page

1. INTRODUCTION 1

1.1 Solid Laminated and Sandwich Structures ........................................................1

1.2 Typical Damage States in Laminated and Sandwich Structures ............................2

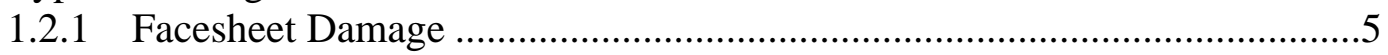

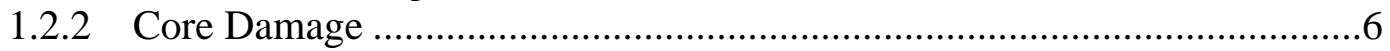

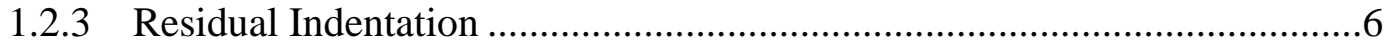

1.3 Behavior of Impact Damaged Sandwich Panels ..............................................8

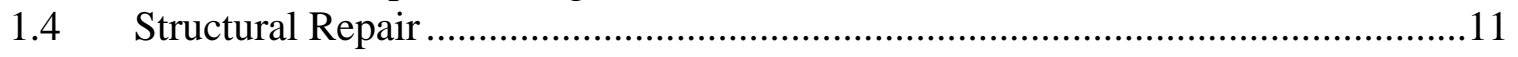

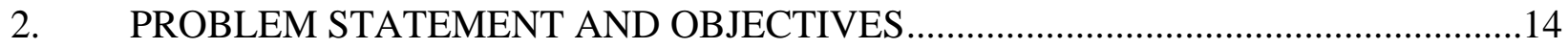

2.1 Statement of Problem................................................................................ 14

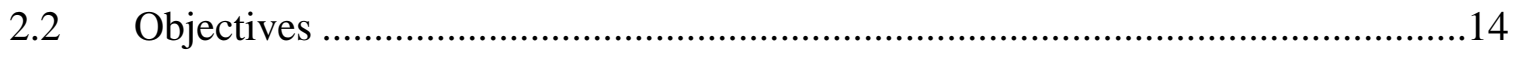

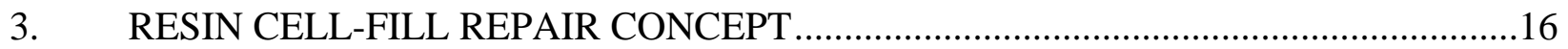

4. MATERIAL SYSTEMS AND EXPERIMENTAL METHODS...............................18

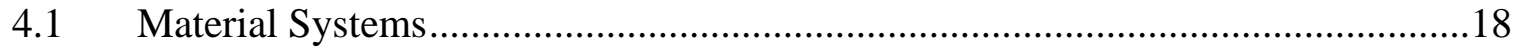

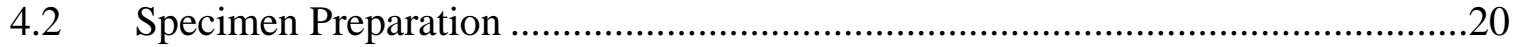

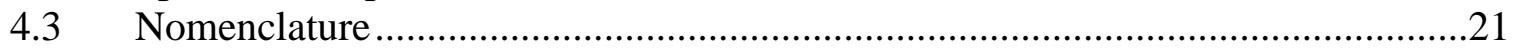

4.4 Creation of Sub-Surface Core Damages ......................................................22

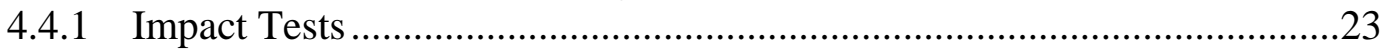

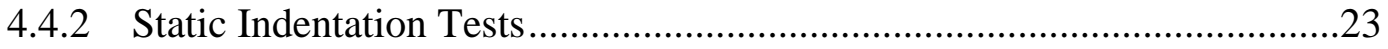

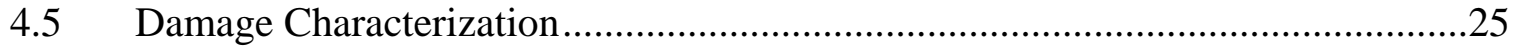

4.5.1 Nondestructive Evaluation of Damage ...............................................25

4.5.2 Destructive Inspection of Damage ....................................................26

4.6 Nondestructive Inspection of Repaired Specimens ........................................27

4.7 Destructive Inspection of Repaired Specimens ..............................................28

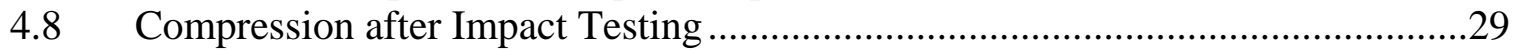

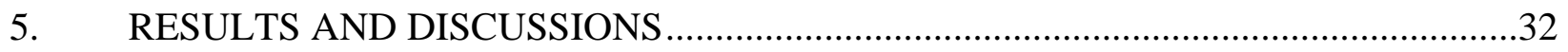

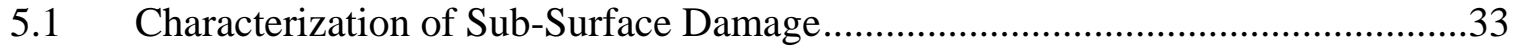

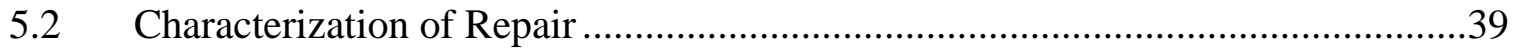

5.3 Characterization of Repair Effectiveness using Edgewise Compression Tests.....44

5.3.1 Measurement of Local Bending ........................................................49

5.3.2 Edgewise Compression Strengths ....................................................54

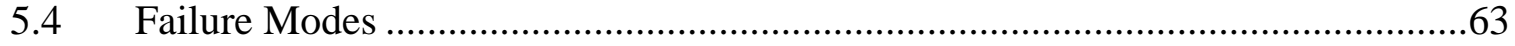


TABLE OF CONTENTS (continued)

Chapter Page

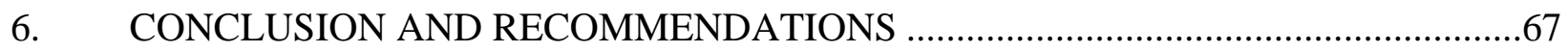

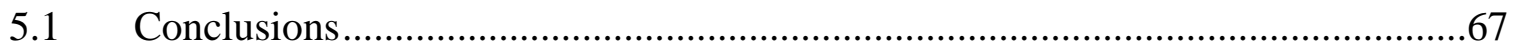

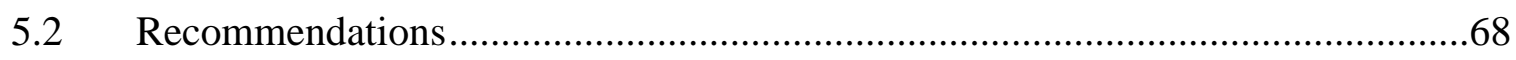

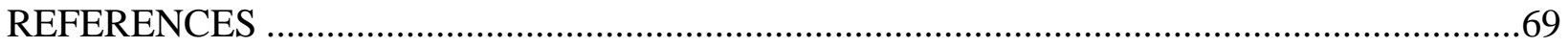

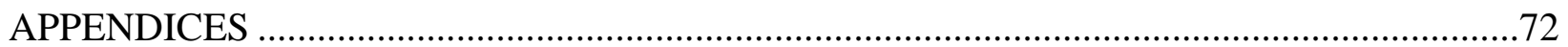

A. End shortening response of S2-FM94, E2-H and CRF specimens .........................73

B. $\quad$ Out-of-plane displacements of S2-FM94, E2-H and CRF specimens ....................78

C. $\quad$ NDI of E2-H Sandwich Specimens with and without repair...................................83

D. NDI of S2-FM94 Sandwich Specimens with and without repair ...........................85

E. NDI of CRF Sandwich Specimens without repair..................................................87

F. NDI of CRF, S2FM94 and E2-H specimens without five-hole repair ...................88 


\section{LIST OF TABLES}

Table

Page

1. Summary of damage metrics and associated degraded properties.............................8

2. Summary of material systems ..................................................................... 19

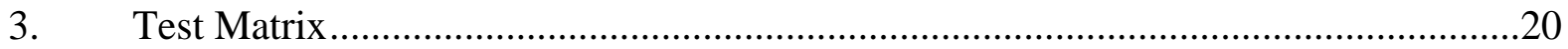

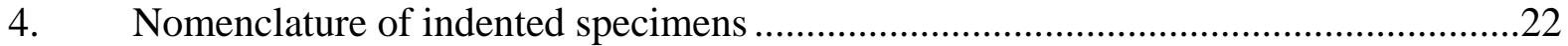

5. Summary of destructive inspections of damaged specimens................................27

6. Summary of destructive inspections of repaired specimens .................................29

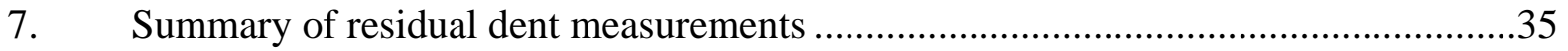

8. Summary of nondestructive inspections of damaged specimens..............................37

9. Summary of nondestructive inspections of repaired specimens ..............................40

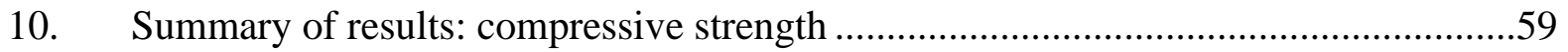

11. Summary of results: max. residual dent depth and planar damage area ....................61

12. Summary of repair effectiveness of different sandwich types...............................62 


\section{LIST OF FIGURES}

Figure

Page

1. Construction Details of 6-ply Solid Laminate .........................................................

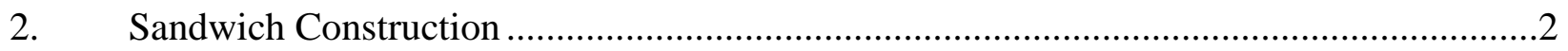

3. Damage Propagation in Thick and Thin Laminates ..................................................5

4. Illustration of core damage and associated damage metrics .......................................6

5. $\quad$ Residual Indentation in Impacted Sandwich Panels ................................................

6. Failure process in sandwich panels with thin-facesheets and

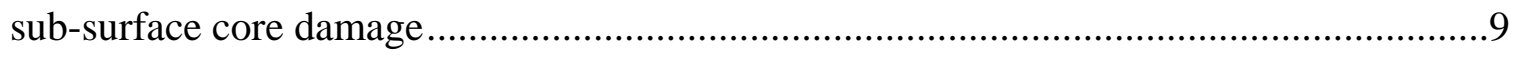

7. Unstable dimple propagation failure $\mathrm{p}$ [rocess in sandwich panels

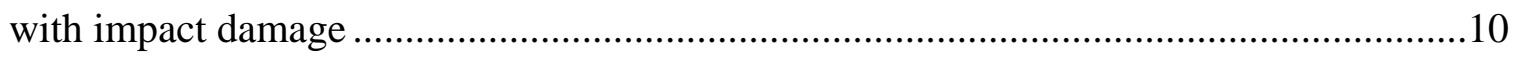

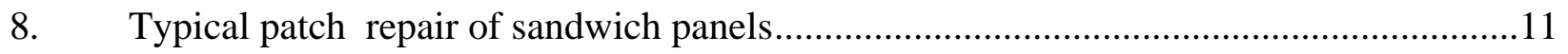

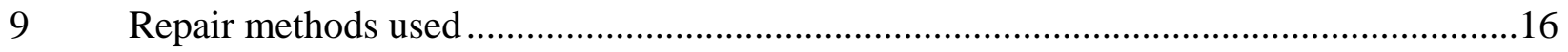

10. Specimens dimensions for S1-FM94 sandwich material system................................21

11. Specimens dimensions for S2-FM94, CRF and E2-H sandwich material systems ...........21

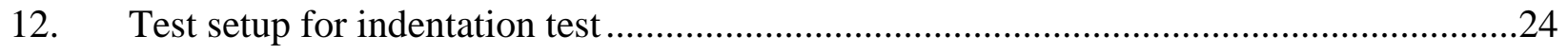

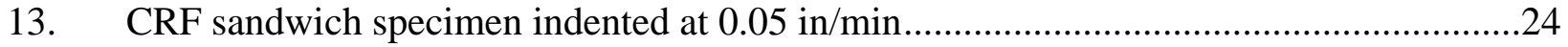

14. TTU C-scan planar area of indented sandwich panel before repair...............................25

15. Dimensions of residual dent obtained by destructive sectioning of

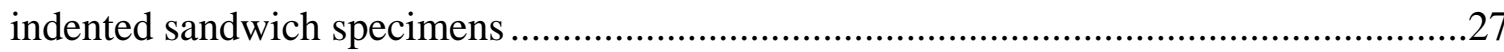

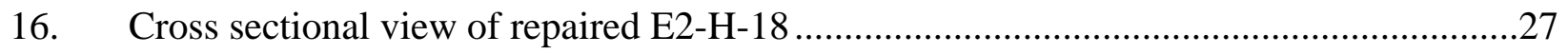

17. TTU C-scan planar damage area of indented sandwich panel after repair ......................28 


\section{LIST OF FIGURES (continued)}

Figure

Page

18. Dimensions of residual dent obtained by destructive sectioning of

repaired sandwich specimens. .28

19. Cross sectional view of repaired specimen (E2-H-17) ............................................29

20. Schematic of compressive residual strength support fixture

with specimen in place.

21. LVDT fixture and LVDT placed at the center of the damage region .30

22. Boundary conditions and unconstrained local out-of-plane displacement along the knife-edges used in compression test.... .32

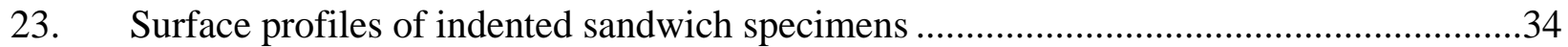

24. TTU C-scan image of CRF-4 (left) and S2-FM94-4 (right) panels with damage .............36

25. TTU C-scan image of S2-FM94-P10 (left) and E2-H-4 (right) panels with damage........36

26. TTU C-scan image of E2-H-12 panel with damage ..................................................36

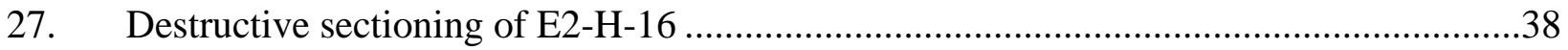

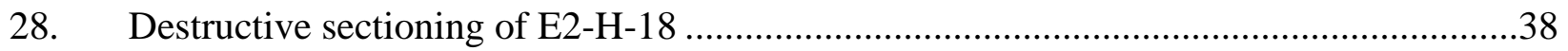

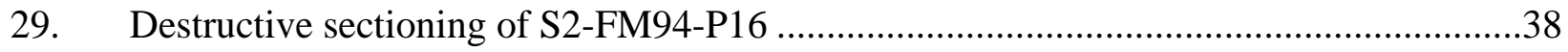

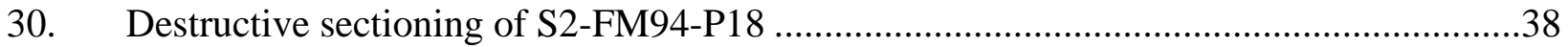

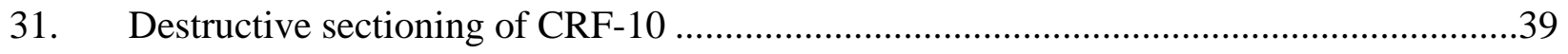

32. TTU C-scan image of S1-FM94-P9-2 with (right) and without (left) 1-Hole Repair .......40

33. TTU C-scan image of S1-FM94-P11-3 with (right) and without (left) 2-Hole Repair .....41

34. TTU C-scan image of S1-FM94-P10-3 with (right) and without (left) 3-Hole Repair .....41

35. TTU C-scan image of CRF-09 with (right) and without (left) 3-Hole Repair. .41 


\section{LIST OF FIGURES (continued)}

Figure

36. TTU C-scan image of S2-FM94-8 with (right) and without (left) 5-Hole Repair............41

37. TTU C-scan image of S2-FM94-15 with (right) and without (left) 5-Hole Repair..........42

38. TTU C-scan image of E2-H-08 with (right) and without (left) 5-Hole Repair ................42

39. TTU C-scan image of E2-H-15 with (right) and without (left) 5-Hole Repair ................42

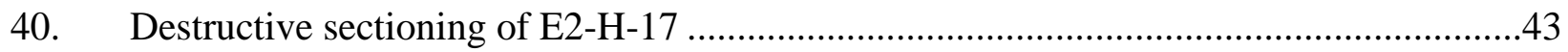

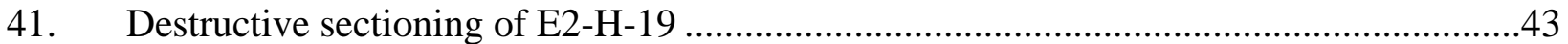

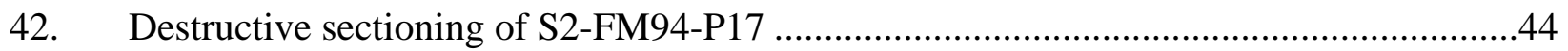

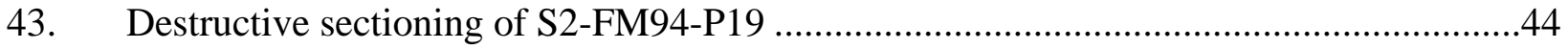

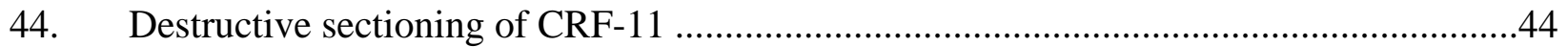

45. Running Load versus End Shortening of S1-FM94 ...............................................46

46. Running load versus end shortening of E2-HX0.10 specimens ..................................47

47. Running load versus end shortening of S2-FM94X0.10 specimens .............................47

48. Running load versus end shortening of CRFX0.15 specimens...................................48

49. Running load versus end shortening of E2-HX0.15 specimens ...................................48

50. Running load versus end shortening of S2-FM94X0.15 specimens .............................49

51. Running load versus out-of-plane displacement of S1-FM94 Sandwich .......................50

52. Running load versus out-of-plane displacement of E2-HX0.10 specimens ....................51

53. Running load verses out-of-plane displacement of S2-FM94X0.10 specimens...............52

54. Running load versus out-of-plane displacement of CRFX0.15 specimens .....................52

55. Running load versus out-of-plane displacement of E2-HX0.15 specimens ....................53

56. Running load versus out-of-plane displacement of S2-FM94X0.15 specimens...............53 


\section{LIST OF FIGURES (continued)}

Figure Page

57. Comparison of Strengths of S1-FM94 sandwich material system.................................55

58. Strength comparisons of CRFX0.15 specimens ........................................................56

59. Strength comparisons of S2-FM94X0.15 specimens................................................56

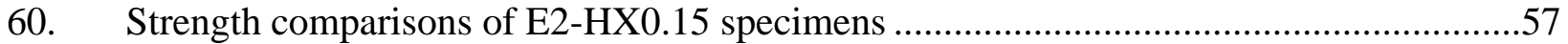

61. Strength comparisons of S2-FM94X0.10 specimens..............................................57

62. Strength comparisons of S2-FM94X0.10 specimens..................................................58

63. Damage growth mechanism and final failure mode observed in damaged

CRF and S2-FM94 sandwich specimens ..........................................................65

64. Different failure modes of specimens with varying number of holes...................66

65. Final failure modes observed in CRF, S2-FM94 and E2-H sandwich

panels for undamaged, damaged and repair configurations 


\section{CHAPTER 1}

\section{INTRODUCTION}

The use of advanced composite materials has been increasing rapidly in a wide range of aerospace applications due to their excellent mechanical properties, such as, high strength to weight ratio, high specific strength, specific stiffness, and fatigue properties.

\subsection{Solid Laminated and Sandwich Structures}

The solid laminates as illustrated in Figure 1 are made up of one or more lamina stacked together and cured at elevated temperatures to form stiffened structure that is lighter than metals. Laminated structures are assembled so that the fiber orientation provides most of the desired mechanical properties and the matrix largely determines the environmental performance.

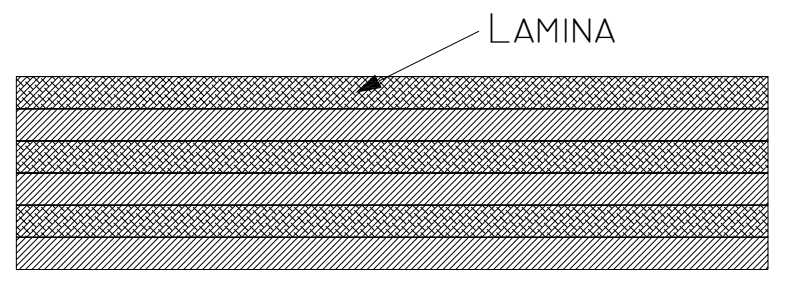

Figure 1: Construction Details of 6-ply Solid Laminate [1]

The concept of using cooperating faces with a distance between them was introduced by Delau in 1820 A.D. and the primitive use of this concept was implemented during World War-II in "Mosquito" aircraft (Veneer Faces and Balsa Wood Core) due to shortage of other materials in England [2]. The purpose of using sandwich structure as shown in Figure 2 is to use a material with a maximum efficiency. The mechanical properties of the sandwich structure can be changed by varying the distance (moment arm) between the two faces of sandwich construction to increase the moment of inertia, thereby increasing the flexural rigidity about the neutral axis of the structure. A sandwich beam of the same width and weight as a solid beam has a higher stiffness due to its higher moment of inertia. 
The sandwich composites act as an I-beam. The facesheets carry in-plane tensile and compressive stresses while the core sustains the transverse shear stresses. The facesheets are stronger than the core which is generally a less dense layer. The honeycomb core material is usually less stiff and has lower strength, which is necessary to achieve the weight reduction. The facesheets are adhesively bonded to the core, or adhere to the core during the co-cure process, depending on the kinds of materials.

The degradation of sandwich panels under loading scenarios can occur at the adhesive bond between the face and the core as well as the node bonds within the honeycomb core itself. There are also other forms of damage, such as, cracking of fillets formed between core and adhesives of sandwich construction, corrosion, and disbond that may be also related to moisture leaking into the panels.

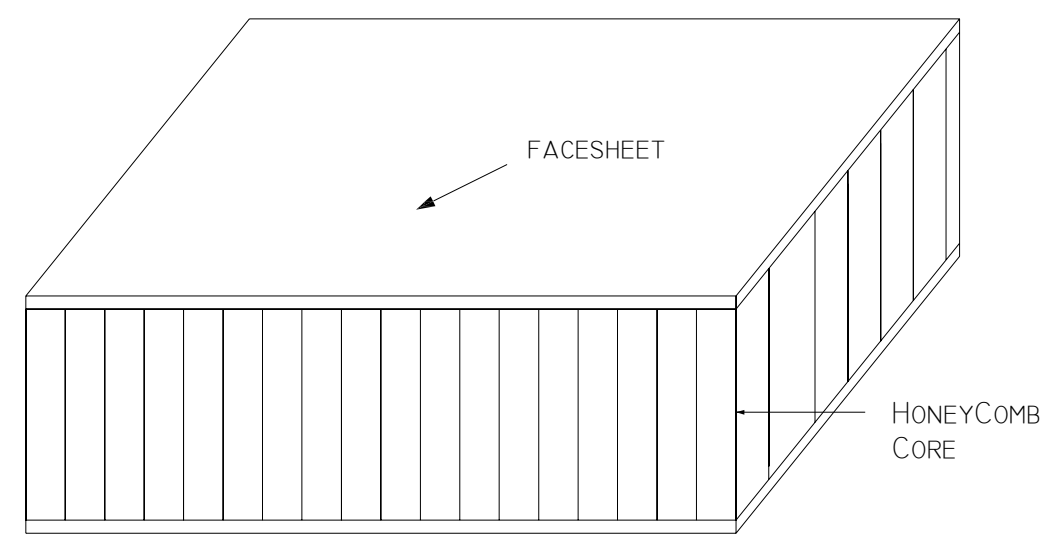

Figure 2: Sandwich Construction [1]

\subsection{Typical Damage States in Laminated and Sandwich Structures [3]}

Most damages that are detrimental to the performance of composite structures are caused by localized transverse impacts. These impacts occur with various combinations of impactor material/geometry, impact velocity, and impact energy. Some examples include tool drop, baggage impact, etc. which occur at low-velocity, and hail-impact, bird impact, run-way debris 
impact etc., which occur at high velocity. Depending upon the energy level associated with the impact event, different damage states can be observed in solid laminates and sandwich structures. The formation of damage due to impact in composite structures is a complex phenomenon. Unlike metals where the energy is dissipated by localized plastic deformation, the composite structures dissipate energy through a combination of sequential failure mechanisms. The impact damage in laminated composites consists of delaminations, fiber failures, matrix cracking, etc. in the immediate vicinity of the impact. At low-energy levels a small dent of the order of ply-thickness may be observed.

However, in sandwich structures, the presence of a weak core underneath the facesheet will produce predominant core damage and an associated residual dent depth. The damage states in sandwich panels can be broadly classified as material damage states and geometric damage states [3]. The material damage states include facesheet damage, core damage, and facesheet-core interface disbonds. The facesheet damage states encompass delaminations, matrix cracks, facesheet fractures, and ply fractures. The core damage states may be comprised of core crushing (foam cores), cell wall buckling (honeycomb cores), and core cell wall fractures. The geometric damage state in sandwich panels manifests as a residual indentation distribution around the point of impact. The various damage states may occur simultaneously with the relative proportions being dictated by the specific combination of intrinsic and extrinsic variables.

Tomblin et al. [5] outlined the parameters defining the impact response affected by impactor size, the damage states due to different impactor sizes, the detection of damage states using traditional inspection techniques and the correlation with the real damage and effects of these damage states on the residual properties. The impacted specimens were subsequently inspected using nondestructive and destructive inspection techniques to characterize the damage. It was observed that the sandwich core of impacted specimens had undergone localized core 
crushing close to the impacted skin over a considerable area. However, the impacted skin, which had not suffered any noticeable damage, thus retaining most of its original stiffness, had retained its state close to original condition. This damage scenario proved to be the most effective when the impacted specimens were inspected using a typical visual inspection method. The effects of various damage states on the performance of the sandwich panels under load were simulated using an edgewise compression test. The compression test results revealed that the damage states due to impact loading with larger impactor (3.0" diameter) behaved as geometric imperfections leading to a local stability governed failure mode. The CAI strength degradation was dominant in case of open-hole type damage states induced by the smaller (1.00" diameter) impactor when compared to that of the larger diameter impactor.

Shipsha et al. [6] investigated the in-plane compressive strength of quasi-isotropic foamcore sandwich panels with core crushing damage. The low-velocity impact scenario was projected to achieve sub-surface core damage followed by residual indentation known as residual dent in the faceplates. The effect of damage states on failure mechanisms in the panels were studied by using digital speckle photography (DSP) analysis, an optical method that measures the whole field displacement along three axes. The impacted panels were tested under displacement controlled edgewise compressive loading condition at a stroke rate of $1 \mathrm{~mm} / \mathrm{min}$. Simple dial gage was used to record the out-of-plane displacements in the center of the residual dent. The CAI test results were finally validated with a finite element model of sandwich panel using ABAQUS to compare the behavior of damaged panels in in-plane compressive loading scenarios. The classical buckling failure leading to abrupt bifurcation was exhibited in undamaged and damaged (low energy levels) sandwich specimens indicating an invisible residual dent growth. The CAI strength was found to decrease with increased impact energy and 
damage size in case of specimens impacted with high energy levels (60 Joules). However, it was observed that the residual strength of panels impacted with 60 Joules was only decreased to $25 \%$ with respect to undamaged specimens.

The damage states observed in impact damaged sandwich panels [3] are summarized in the following sections.

\subsubsection{Facesheet Damage}

The facesheet damage states may be comprised of facesheet delaminations, matrix cracking, facesheet fractures, and ply fractures. The initiation of facesheet damage depends on the shape and size of impactor. The facesheet damage initiates in the form of delaminations between the laminas adjacent to the facesheet-core interface and these delaminations occurred above the honeycomb cell walls. Increasing impact load induces a network of delaminations. When the thick solid laminate is subjected to an impact loading on the top as illustrated in Figure 3, the damage propagates in the form of a tree in a uniformly distributed way. Due to the stress concentration on the top most lamina of solid thick laminate, the damage known as delamination gets distributed propagating the delamination. However, the damage propagation in thin laminates is different. As shown below in Figure 3, the thin solid laminate when subjected to impact loads has inverted delamination pattern as compared to thick laminates.
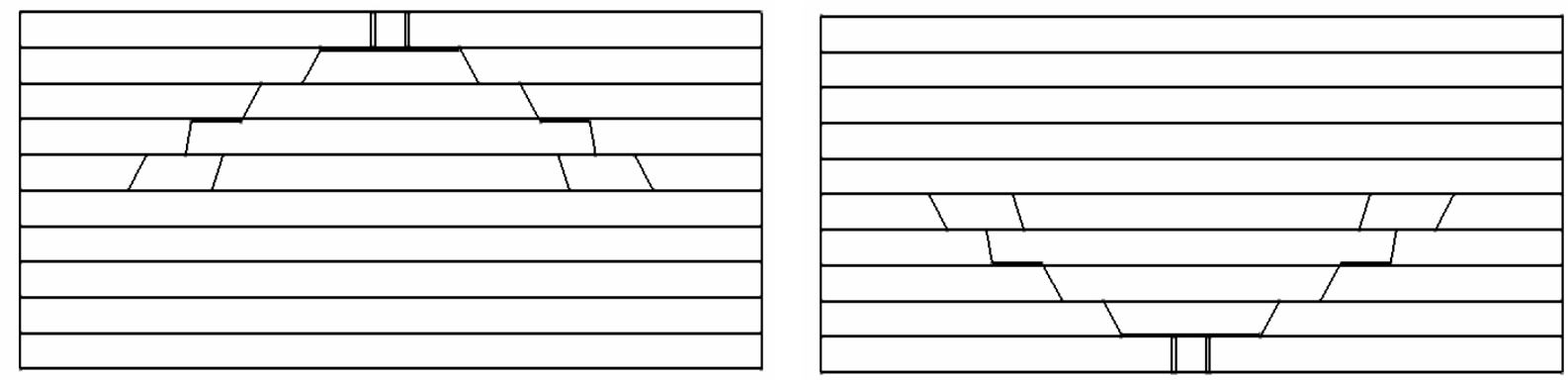

Figure 3: Damage propagation in thick (left) and thin (right) laminates [4] 


\subsubsection{Core Damage}

The core damage in honeycomb core sandwich panels results in cell wall buckling, core crushing, and cell wall fracture. The cell wall buckling propagates across the planar dimensions of the specimen indicating an incipient failure mode in all cases. The planar damage size $2 \mathrm{R}_{\text {damage }}$ of the sandwich panel may be captured by Through Transmission Ultrasonic (TTU) C-scan. The TTU C-scan images of sandwich panels tend to correlate better with core damage rather than facesheet damage as the damaged core attenuates the ultrasonic signals more than the facesheets. In addition to this, the destructive sectioning of damaged sandwich panels is used to characterize the effects of facesheet stiffness and core properties on the core crush depths associated with planar damage size. The ratio of planar damage size $\left(2 \mathrm{R}_{\text {damage }}\right)$ to the maximum crush depth $\left(\Lambda_{\text {crush }}\right)$, in general, depends on the impactor size, facesheet stiffness, and transverse compressive behavior of the core. The damage metrics associated with the core damage in honeycomb sandwich panels are illustrated in Figure 4.

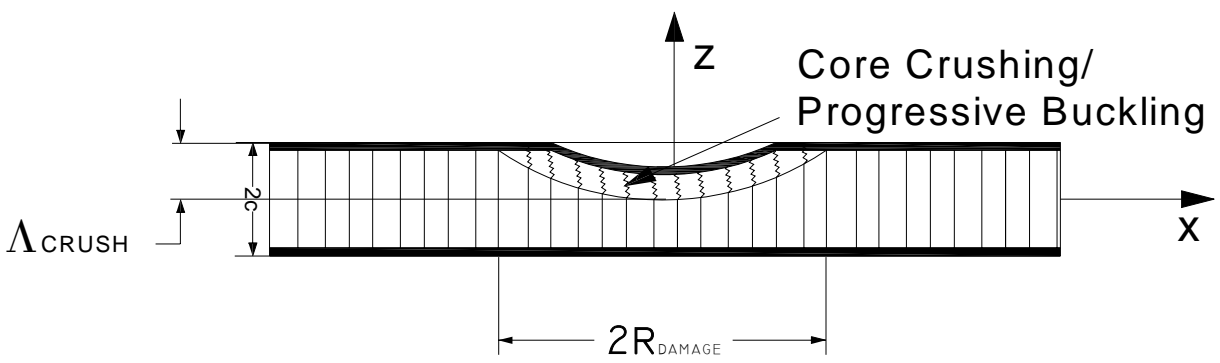

Figure 4: Illustration of core damage and associated damage metrics for honeycomb core sandwich

\subsubsection{Residual Indentation} panels [3]

The damage states in sandwich panels are confined to the facesheet and the core. However, due to the structural interaction between the damaged components of the sandwich panels, a geometric imperfection exists in impact-damaged sandwich panels. Upon removal or cessation of the indenting/impacting force, the facesheet tends to return to its original undamaged 
position. However, the damaged core resists the facesheet (which could be damaged) from returning to its original position. The equilibrium position between the facesheet and damaged core results in a zone of depression referred to as residual indentation as illustrated in Figure 5. The residual indentation distribution is characterized by the maximum residual indentation depth $\Lambda_{\text {RMAX }}$ occurring at the center of the indentation region (point of impact) and the planar size $2 \mathrm{R}_{\text {ind }}$ of the residual indentation.

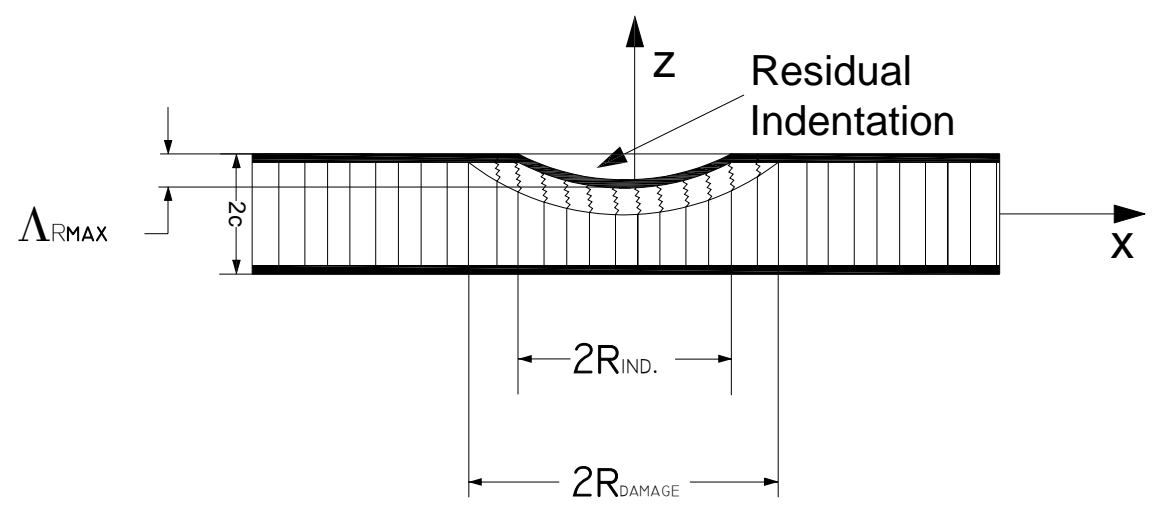

Figure 5: Residual indentation in impacted sandwich panels [3]

The $\Lambda_{\mathrm{RMAX}}$, for a given planar damage size of the core $2 \mathrm{R}_{\text {damage }}$, depends on the facesheet stiffness and the tensile properties of the damaged core. The residual indentation depends on the thickness of the facesheet and magnitude of impact load. Also, the core that undergoes progressive buckling when subjected to impact loads is not sufficient to provide tensile resistance to the spring nature of facesheet, further reducing the residual indentation and resulting barely visible impact damage. The damage metrics associated with the sandwich components, quantification methods, and associated degraded properties are summarized in Table 1. 
TABLE 1

SUMMARY OF DAMAGE METRICS AND ASSOCIATED DEGRADED PROPERTIES [3]

\begin{tabular}{|c|c|c|c|c|}
\hline $\begin{array}{l}\text { Sandwich } \\
\text { Component }\end{array}$ & Damage Metrics(s) & $\begin{array}{l}\text { Measurement } \\
\text { Method }\end{array}$ & $\begin{array}{c}\text { Degraded } \\
\text { Properties }\end{array}$ & Comments \\
\hline Facesheet & $\begin{array}{l}\text { Planar facesheet damage size } \\
\qquad 2 \mathrm{R}_{\text {skin }}\end{array}$ & $\begin{array}{c}\text { Visual, } \\
\text { Destructive } \\
\text { sectioning } \\
\end{array}$ & $\begin{array}{l}\text { Facesheet } \\
\text { stiffness } \\
\text { (flexural) }\end{array}$ & $\begin{array}{l}\text { Region not } \\
\text { necessarily } \\
\text { circular } \\
\end{array}$ \\
\hline \multirow{2}{*}{ Core } & $\begin{array}{l}\text { Planar core damage size } \\
2 \mathrm{R}_{\text {damage }} \\
\end{array}$ & NDI & \multirow{2}{*}{$\begin{array}{l}\text { Transverse } \\
\text { properties of } \\
\text { core }\end{array}$} & \multirow{2}{*}{$\begin{array}{l}\text { Region not } \\
\text { necessarily } \\
\text { circular }\end{array}$} \\
\hline & $\begin{array}{c}\text { Maximum Core Crush depth } \\
\Lambda_{\text {crush }}\end{array}$ & $\begin{array}{l}\text { Destructive } \\
\text { sectioning }\end{array}$ & & \\
\hline \multirow{2}{*}{ Facesheet } & $\begin{array}{l}\text { Maximum residual } \\
\text { indentation } \Lambda_{\mathrm{RMAX}}\end{array}$ & $\begin{array}{l}\text { Coordinate } \\
\text { measurement } \\
\text { machine }\end{array}$ & \multirow{2}{*}{ N/A } & \multirow{2}{*}{$\begin{array}{l}\text { Region not } \\
\text { necessarily } \\
\text { circular }\end{array}$} \\
\hline & $\begin{array}{l}\text { Planar size of residual } \\
\text { indentation region } 2 \mathrm{R}_{\text {ind }}\end{array}$ & $\begin{array}{c}\text { Coordinate } \\
\text { measurement } \\
\text { machine } \\
\end{array}$ & & \\
\hline Interface & $\begin{array}{l}\text { Residual traction field on } \\
\text { facesheet due to damaged } \\
\text { core }\end{array}$ & Analytical model & N/A & N/A \\
\hline
\end{tabular}

\subsection{Behavior of Impact Damaged Sandwich Panels}

The damage tolerance of laminated and sandwich structures with impact damage has been investigated previously [3], under in-plane compressive loads. The behavior of sandwich panels with impact damage was observed to be dictated by the amount of underlying core damage accompanied by residual dent. The sandwich panels with visible facesheet damage characteristic of an impact with small diameter impactor produced failure modes under in-plane compressive loading that resembled a net-section fracture. In addition, sandwich panels with thin facesheets and subsurface core damage exhibited a net-section fracture which was precipitated by the local bending of the facesheet as illustrated in Figure 6. The in-plane compressive loads bend the skin within the damage zone due to the lack of support from the damaged core. This local bending deformation increases until the strain in the skin exceeds a critical strain value initiating skin fractures at the edge of the damage region. These cracks propagate out laterally, 
towards the edges of the specimen leading to complete fracture. This failure process is illustrated in Figure 6.

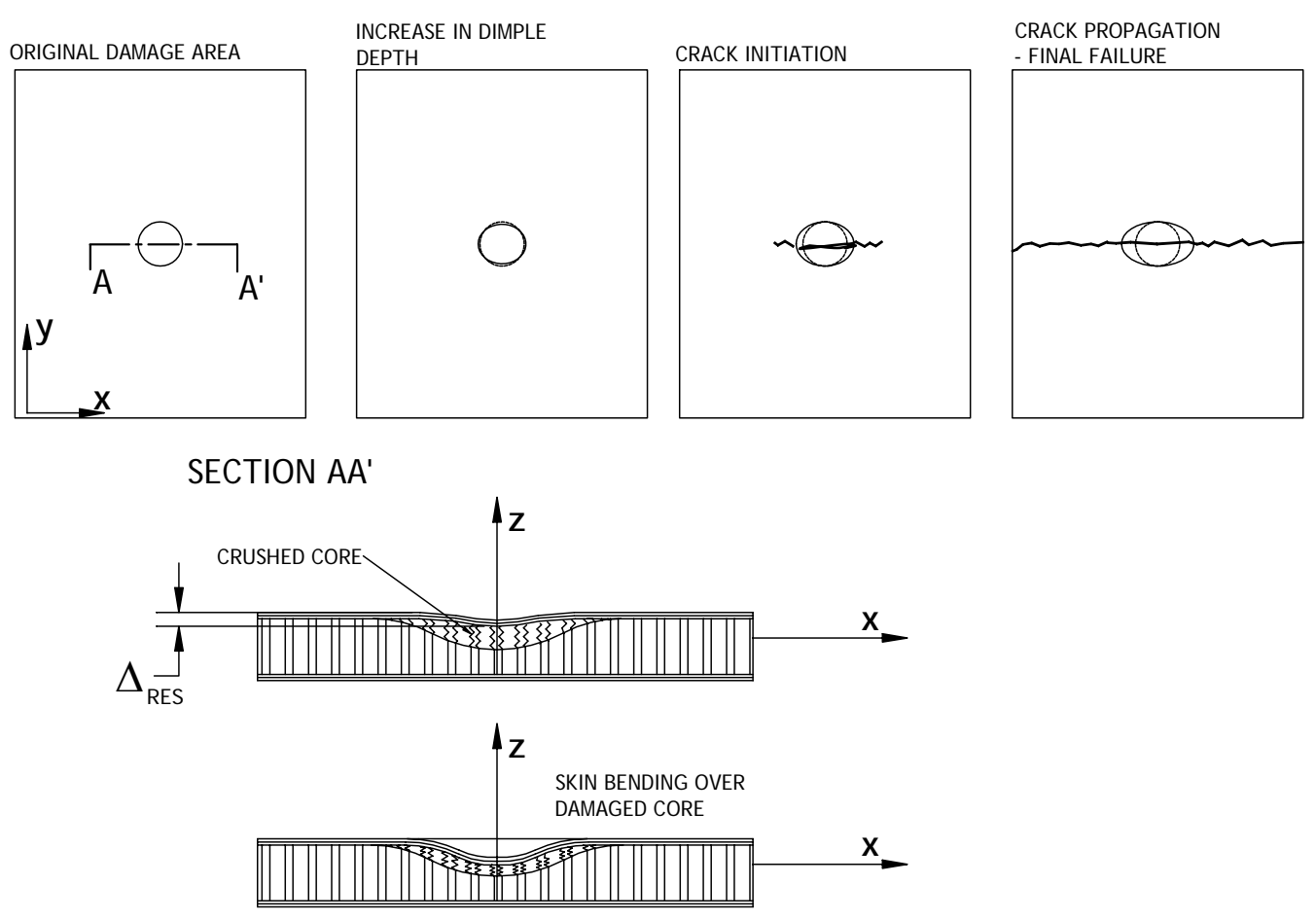

Figure 6: Failure process in sandwich panels with thin-facesheets and sub-surface core damage [3]

The sandwich panels with impact damage due to larger diameter impactors, with little or no facesheet damage but considerable core damage, produced failure modes dominated by localized instability. Similar to the previous case, the local bending of facesheet occurs over the damaged core, during the initial loading. However, the facesheet accumulates enough energy to initiate crushing of additional core and further increase the local compliance. This initial core damage growth will be arrested and further local bending of facesheet occurs. Upon further loading, the energy accumulated in the facesheet is high enough to precipitate unstable dimple propagation across the width of the core, crushing the undamaged core in the process. This 
failure mechanism is illustrated in Figure 7 and was prominent in sandwich panels with thicker facesheets and subsurface core damage. The reduced compressive strength due to this failure mechanism has been reported to approach $40 \%$ of the undamaged strength and thus detrimental to the safe operation of the structure.
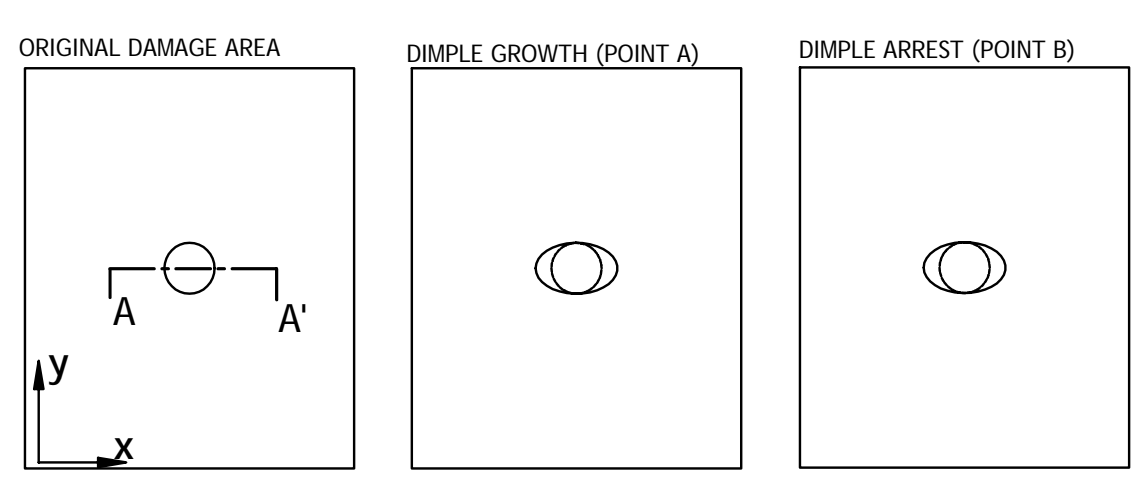

UNSTABLE DIMPLE GROWTH

-FINAL FAILURE (POINT C)
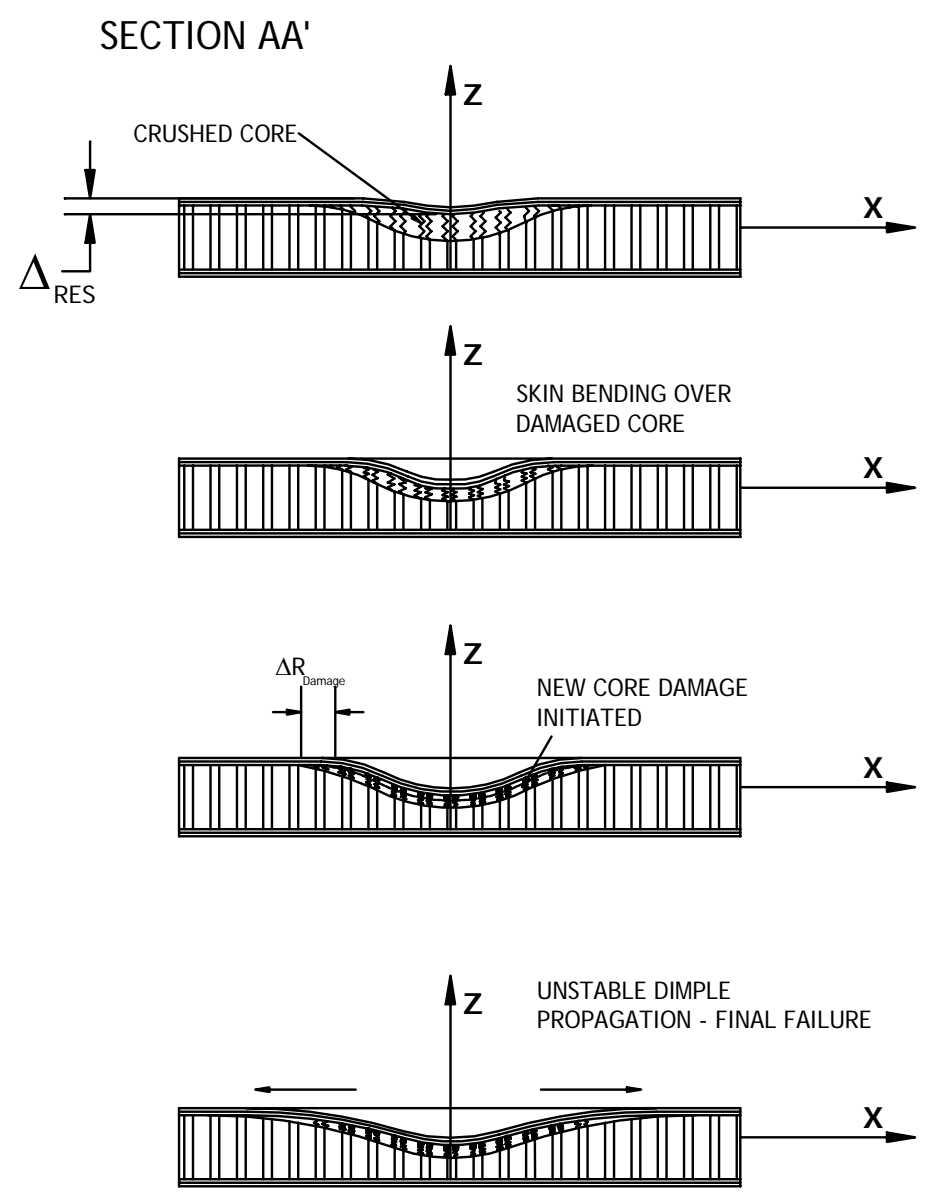

Figure 7: Unstable dimple propagation failure process in sandwich panels with impact damage [3] 


\subsection{Structural Repair}

The increasing use of sandwich composite materials in aircraft primary structures requires the development of proven repair methods that restore the integrity of the damaged structure, with minimum weight addition. The typical repair procedures for sandwich structures involve removal of the damaged facesheet(s) and core over a region slightly larger than the original planar damage size. The edges of the removed facesheet are then tapered/scarfed appropriately to facilitate a scarf joint. The damaged region is repaired by inserting a new core plug and facesheet plies that are bonded to the original sandwich structure using an adhesive layer. The repair procedure is illustrated in Figure 8 below.

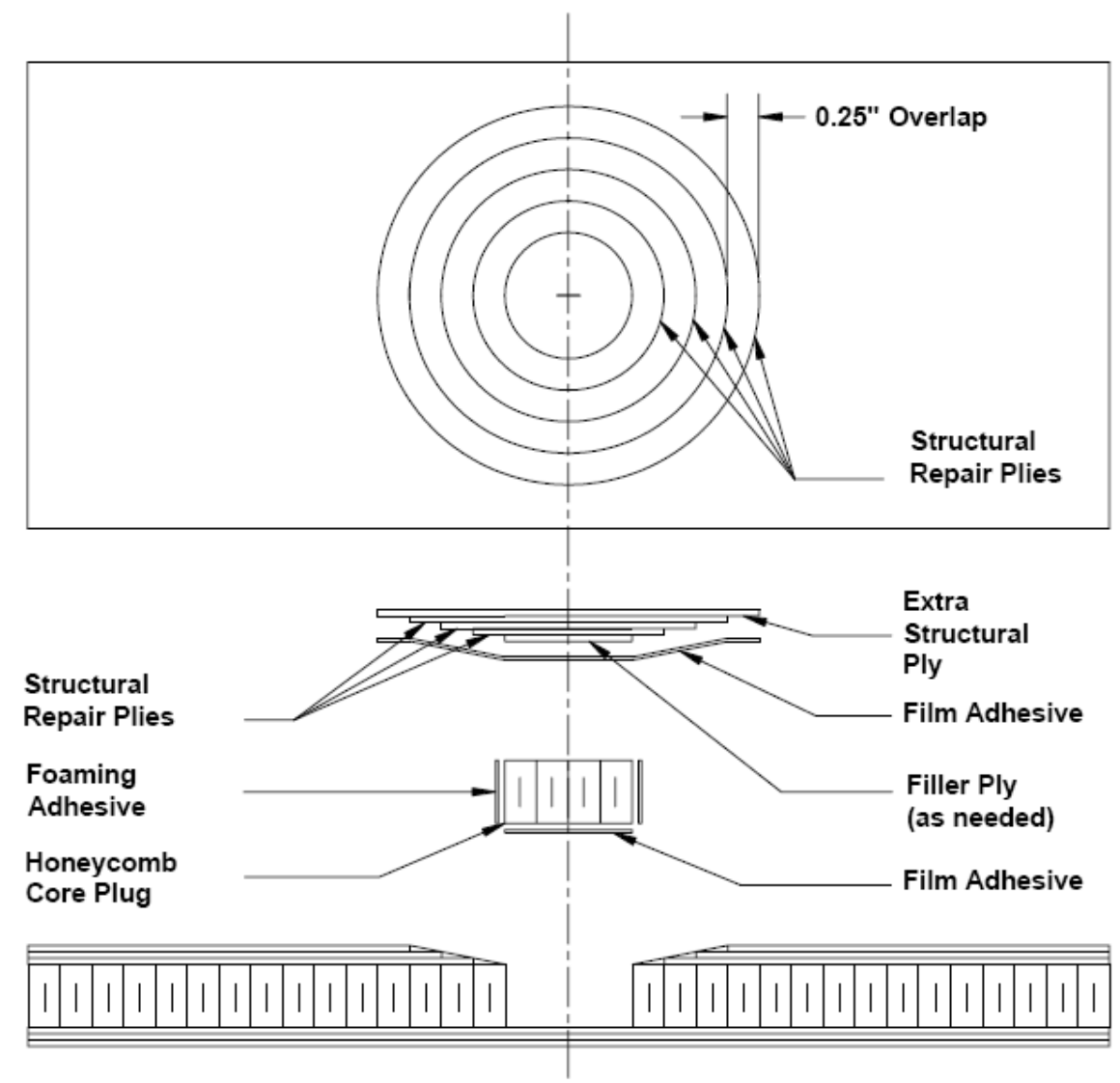

Figure 8: Typical patch repair of sandwich panels [8] 
The effectiveness of scarf repairs applied to sandwich structures was evaluated by Tomblin et al [8] using several bonding repair variables. These variables include three scarf overlaps $\left(0.15^{\prime \prime}, 0.25^{\prime \prime}\right.$ and $\left.0.50^{\prime \prime}\right)$, two different core cell sizes (1/8" and $\left.3 / 8^{\prime \prime}\right)$, curing temperature conditions and the consequences of thermal stresses induced by thermal conditioning of the patch repairs. The effects of wet lay-up procedure and prepreg procedure repair methods comprising of different cure temperatures and materials were highlighted. The wet lay-up procedure was thicker than prepreg procedure method. The prepreg method used a steeper 0.25 " scarf overlap while the wet lay-up method utilized a conventional 0.50 " scarf overlap. However, the strength of the repaired specimens was improved to $92 \%$ of the average undamaged value in both types of repair methods. Unidirectional tension test and, small and large four-point flexure tests were conducted to evaluate the effect of different variables on repair performance. The strength of steeper scarf $\left(0.15^{\prime \prime}\right)$ repaired specimens was found to be decreased with respect to other scarf repairs due to the presence of high stress concentration in adhesive bondline leading to final failure of the specimens.

M. Raju et al. [7] investigated the repair effectiveness of inexpensive wet repair patches on polyurethane foam core (PUF) and aramid honeycomb core (Nomex®) sandwich specimens with no damage, impact damage and damage with repair, using edgewise compression and flexure tests. The repair effectiveness defined as the ratio of difference between repair and damaged strengths to the difference between virgin and damaged strengths was used to evaluate the repair. The repair was observed to be more effective ( 0.5 to 0.81 ) in honeycomb core sandwich specimens when compared to the foam core specimens. The strength recoveries of up to $90 \%$ in PUF core and $88 \%$ in honeycomb core sandwich specimens were realized after repair. 
Further, the repairs performed better under flexure tests when compared to the edgewise compression tests.

$$
\text { repair effectiveness }=\frac{\text { strength of repaired specimen }- \text { strength of damaged specimen }}{\text { strength of undamaged specimen }- \text { strength of damaged specimen }}
$$

The scarf repair of the sandwich has been shown to restore the strength of the structure to levels approaching that of the undamaged panels. The key to the success of the repair is the selection of the scarf angle to minimize the peel stresses on the adhesive joint and the selection of the stiffness of the repair patch to strike a good balance between the load being transmitted into the patch via the adhesive layer and the load bypassing the repair region. This repair method is sensitive to the quality of the scarf surfaces used for bonding and is labor intensive.

The damage states in honeycomb sandwich panels impacted by blunt impactors are primarily one consisting of damaged core cells and associated residual dent. The facesheets suffer little or no damage in these scenarios. Thus, performing the aforementioned patch repair may be too invasive, expensive and could be overkill for the problem at hand. Since the unstable dimple propagation triggered by local facesheet bending over the damaged core is the cause of reduced strengths of sandwich panels under compression, a repair method focused on providing localized core stabilization may be simpler. The present study involves an experimental investigation of a simple repair technique where the damage cell walls are strategically reinforced to counter the local bending of the facesheet and thus delay or eliminate the unstable dimple propagation failure mechanism. The details of the different repair configurations, methods used to characterize their effectiveness and the experimental results and observations are presented in this report. 


\section{CHAPTER 2}

\section{PROBLEM STATEMENT AND OBJECTIVES}

\subsection{Statement of Problem}

Under in-plane compressive loads, the key failure mechanism in sandwich panels with subsurface core damage is by local bending of facesheet over the damaged core. This local bending triggers an unstable dimple propagation resulting in loss of load carrying capability. While the patch repair method is effective in restoring the strength, the same could be accomplished by containing the local bending of facesheet; thereby delaying or eliminating the triggering of the unstable propagation of the dimple. The problem involves identifying the strategic locations in the damage zone, where the damaged core cells need to be reinforced to reap the maximum benefits without paying a severe weight penalty

\subsection{Objectives}

The main objective of this research is to contain the local bending of subsurface core damaged sandwich panels by introducing a resin repair concept. This research aims to characterize the repair effectiveness of impacted (or indented) sandwich specimens using in-plane compression tests. The effectiveness of the repair will be characterized in terms of:

- Compressive Residual strength of sandwich panels

- Amount of out-of-plane deformation in the damaged region under in-plane compressive loads ( far-field)

- $\quad$ Failure modes

- Far field strains

The repair concept will be exercised on different sandwich facesheet-core combinations to ensure broad applicability of the concept. The subsurface damage will be generated using 
low-velocity impact / static indentation methods. The details of the material systems, experimental procedures, observations and results are documented in this report. 


\section{CHAPTER 3}

\section{RESIN CELL-FILL REPAIR CONCEPT}

In order to significantly reduce the replacement cost of the aircraft composite structures during routine maintenance, a novel repair method has been proposed. The repair method will strategically provide through the thickness reinforcement to the damaged region of the sandwich structure. The resin repair concept was chosen in this investigation due to its ease of handling and cost effectiveness aspects. A repair technique not common in aerospace repair methods, involving the containment of local bending of sandwich facesheet by strategically filling honeycomb core cells has been explored to improve the damage tolerance of impact damaged sandwich panels.

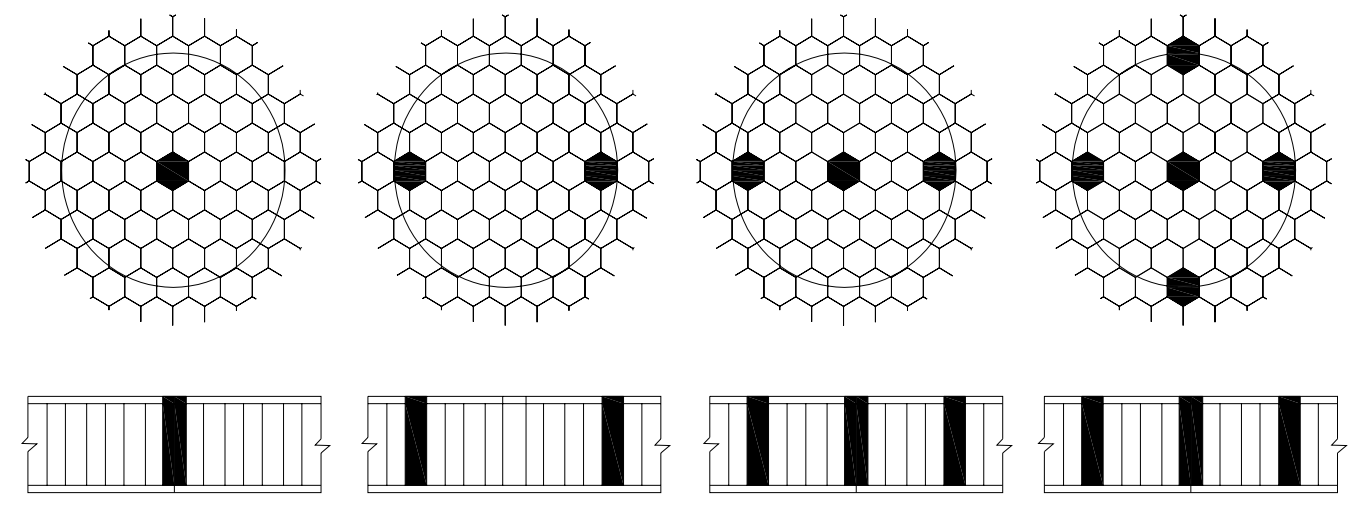

Figure 9: Repair methods used

Four repair configurations such as one-hole, two-hole, three-hole and five-hole used in this investigation are illustrated in Figure 9. The size of the repaired hole signifies the actual size of honeycomb core cell. Sandwich specimens were repaired by drilling 0.125 " diameter holes and filling the honeycomb cell(s) with a mixture of Epoxy EPON resin and hardener [13]. A total of five holes, four on the circumference and the last one at the center of the circle were chosen as 
per five-hole repair shown in Figure 9. The resin system comprises of EPON-Resin 828 (contains: Bisphenol A Epoxy Resin CAS 25068-38-6) and EPI-CURE-3274 Curing Agent (contains Polyoxyalkyleneamine 9046-10-0) in the ratio of 100:40 by parts. All the repair schemes were cured at room temperature for a minimum of twenty four hours. Filling all the five drilled holes (Figure 9) with resin mixture reinforces the damaged cell walls in addition to providing out-of-plane support. 


\section{CHAPTER 4}

\section{MATERIAL SYSTEMS AND EXPERIMENTAL METHODS}

This chapter provides the details of the material systems used for sandwich panels, lay-up sequences and fabrication methods. The methods and apparatus used for creating the subsurface core damage and its characterization using on-destructive and destructive methods are discussed. The details of the compression testing to characterize the severity of the damage and the effectiveness of the repair are presented in this chapter.

\subsection{Material Systems}

A total of four sandwich configurations were selected to address a range of facesheets and core thickness combinations. The facesheet material systems, stacking sequences, adhesives and core details are summarized in Table 2. The honeycomb core used in the present study had a nominal cell size of $0.1875 "$ and a nominal density of $3 \mathrm{lb} / \mathrm{ft}^{3}$. The facesheet material systems comprised of CYCOM 7714A/5HS T650-35 Carbon Fabric/Epoxy prepreg [10], Hexcel 8552 Carbon Fabric/Epoxy prepreg [12] and NB321/3K70P Carbon Fabric/Epoxy prepreg [11].

The facesheets were bonded to cores using a co-cure process to fabricate sandwich panels with nominal planer dimensions of 43" by 12". The adhesives used were CYTEC FM-94 Film Adhesive [10], AF163 Adhesive Film [10] and HYSOL 9628.060 PSF NW Film Adhesive [17]. 
TABLE 2

SUMMARY OF MATERIAL SYSTEMS

\begin{tabular}{|c|c|c|c|c|}
\hline $\begin{array}{l}\text { Sandwich } \\
\text { Type }\end{array}$ & S1-FM94 & S2-FM94 & E2-H & CRF \\
\hline $\begin{array}{l}\text { Facesheet } \\
\text { Material }\end{array}$ & \multicolumn{2}{|c|}{$\begin{array}{l}\text { CYCOM 7714A/5HS T650-35 } \\
\text { Carbon Fabric/Epoxy prepreg [10] }\end{array}$} & $\begin{array}{l}\text { Hexcel } 8552 \\
\text { Carbon } \\
\text { Fabric/Epoxy } \\
\text { prepreg [12] }\end{array}$ & $\begin{array}{c}\text { NB321/3K70P } \\
\text { Carbon Fabric/Epoxy } \\
\text { prepreg [11] }\end{array}$ \\
\hline Weave Pattern & \multicolumn{2}{|c|}{5 Harness-Satin Weave } & \multicolumn{2}{|c|}{ Plain Weave } \\
\hline $\begin{array}{l}\text { Stacking } \\
\text { Sequence }\end{array}$ & {$[(45 / 45) /$ core $] \mathrm{s}$} & {$[(45 / 0 / 45) /$ core $] \mathrm{s}$} & $\begin{array}{c}{[45 / 0 / 45 / 904 / 4} \\
5 / 0 / 45 / \text { core } / 45 \\
/ 0 / 452 / 0 / 45]\end{array}$ & {$[(90 / 45) /$ core $] \mathrm{s}$} \\
\hline Core Material & \multicolumn{3}{|c|}{ Hexcel N636 Honeycomb [12] } & $\begin{array}{c}\text { Plascore Nomex }{ }^{\circledR} \\
\text { Honeycomb Cores } \\
(\mathrm{PN2}-3 / 16-4.5 .0) \\
\end{array}$ \\
\hline $\begin{array}{l}\text { Core } \\
\text { Thickness }\end{array}$ & \multicolumn{2}{|c|}{$0.50^{\prime \prime}$} & $0.25^{\prime \prime}$ & $0.75^{\prime \prime}$ \\
\hline Adhesive & \multicolumn{2}{|c|}{ Cytec FM94 Adhesive Film [10] } & $\begin{array}{c}\text { AF163 } \\
\text { Adhesive } \\
\text { Film [10] }\end{array}$ & $\begin{array}{c}\text { Hysol EA } 9628.060 \\
\text { PSF NW Film } \\
\text { Adhesive [17] }\end{array}$ \\
\hline
\end{tabular}

Table 3 defines the test matrix used in this investigation in which S1-FM94 specimens were impacted while the remaining sandwich materials listed in test matrix were statically indented to create subsurface core damage. The static indentation was preferred as it offers better control over the creation of damage. Three sandwich configurations: undamaged, damaged and damaged with repair were chosen in case of S2-FM94, CRF and E2-H sandwich materials. Five configurations namely; undamaged, damaged, damage with one-hole repair, damage with twohole repair, damage with three-hole repair were selected to evaluate the locations and number of holes required for resin repair for S1-FM94 sandwich specimens. This particular sandwich configuration was used to study the effectiveness of different repair configurations. Based on the findings of this study, a single repair configuration was chosen for the remainder of the sandwich 
specimens. Three specimens in each configuration (i.e., undamaged, damaged and repaired) were tested. One specimen in damaged and repaired configurations was selected for destructive sectioning for S2-FM94, CRF and E2-H sandwich specimens.

TABLE 3

TEST MATRIX

\begin{tabular}{|c|c|c|c|c|c|c|}
\hline \multirow{2}{*}{$\begin{array}{l}\text { Panel } \\
\text { Type }\end{array}$} & \multirow{2}{*}{$\begin{array}{l}\text { Damage } \\
\text { Type }\end{array}$} & \multirow[b]{2}{*}{ Configurations } & \multirow{2}{*}{$\begin{array}{c}\text { Impact Energy/ } \\
\text { Indentation Depth }\end{array}$} & \multicolumn{3}{|c|}{ No. of Specimens } \\
\hline & & & & $\begin{array}{l}\text { CAI } \\
\text { Test }\end{array}$ & $\begin{array}{l}\text { Destructive } \\
\text { Inspection }\end{array}$ & Total \\
\hline \multirow{5}{*}{ S1-FM94 } & $\mathrm{N} / \mathrm{A}$ & Undamaged & $\mathrm{N} / \mathrm{A}$ & 3 & $\mathrm{~N} / \mathrm{A}$ & \multirow{5}{*}{19} \\
\hline & \multirow{4}{*}{ Impact } & Damaged & 40 in-lbs & 3 & 1 & \\
\hline & & Repair-1Hole & 40 in-lbs & 3 & 1 & \\
\hline & & Repair-2 Holes & 40 in-lbs & 3 & 1 & \\
\hline & & Repair-3 Holes & 40 in-lbs & 3 & 1 & \\
\hline \multirow{5}{*}{ E2-H } & N/A & Undamaged & $\mathrm{N} / \mathrm{A}$ & 3 & N/A & \multirow{5}{*}{19} \\
\hline & \multirow{4}{*}{ Indentation } & \multirow{2}{*}{ Damaged } & $0.10^{\prime \prime}$ & 3 & 1 & \\
\hline & & & $0.15^{\prime \prime}$ & 3 & 1 & \\
\hline & & \multirow{2}{*}{ Repair-5 Holes } & $0.10^{\prime \prime}$ & 3 & 1 & \\
\hline & & & $0.15^{\prime \prime}$ & 3 & 1 & \\
\hline \multirow{5}{*}{ S2-FM94 } & N/A & Undamaged & N/A & 3 & N/A & \multirow{5}{*}{19} \\
\hline & \multirow{4}{*}{ Indentation } & \multirow{2}{*}{ Damaged } & $0.10^{\prime \prime}$ & 3 & 1 & \\
\hline & & & $0.15^{\prime \prime}$ & 3 & 1 & \\
\hline & & \multirow{2}{*}{ Repair-5 Holes } & $0.10^{\prime \prime}$ & 3 & 1 & \\
\hline & & & $0.15^{\prime \prime}$ & 3 & 1 & \\
\hline \multirow{3}{*}{$\mathrm{CRF}$} & N/A & Undamaged & N/A & 3 & N/A & \multirow{3}{*}{11} \\
\hline & \multirow{2}{*}{ Indentation } & Damaged & $0.15^{\prime \prime}$ & 3 & 1 & \\
\hline & & Repair-5 Holes & $0.15^{\prime \prime}$ & 3 & 1 & \\
\hline
\end{tabular}

\subsection{Specimen Preparation}

After autoclave curing of sandwich panels, the panels (46 inches wide and 12 inches tall) were cut using a table saw to obtain two sub-panels which were then trimmed into rectangular specimen size of 10.50 inches tall and 4.20 inch wide as illustrated in Figure 10 (S1-FM94 specimens) and 10.50 inches tall and 4.00 inches wide as shown in Figure 11 (E2-H, S2-FM94 and CRF specimens) in a coolant lubricated precision cutting and were finally grinded in a dry 
grinding mode (no coolant). During the machining process, the specimens were appropriately supported to mitigate the probability of delamination and/or matrix cracking. The specimens were then dimensioned using vernier caliper.

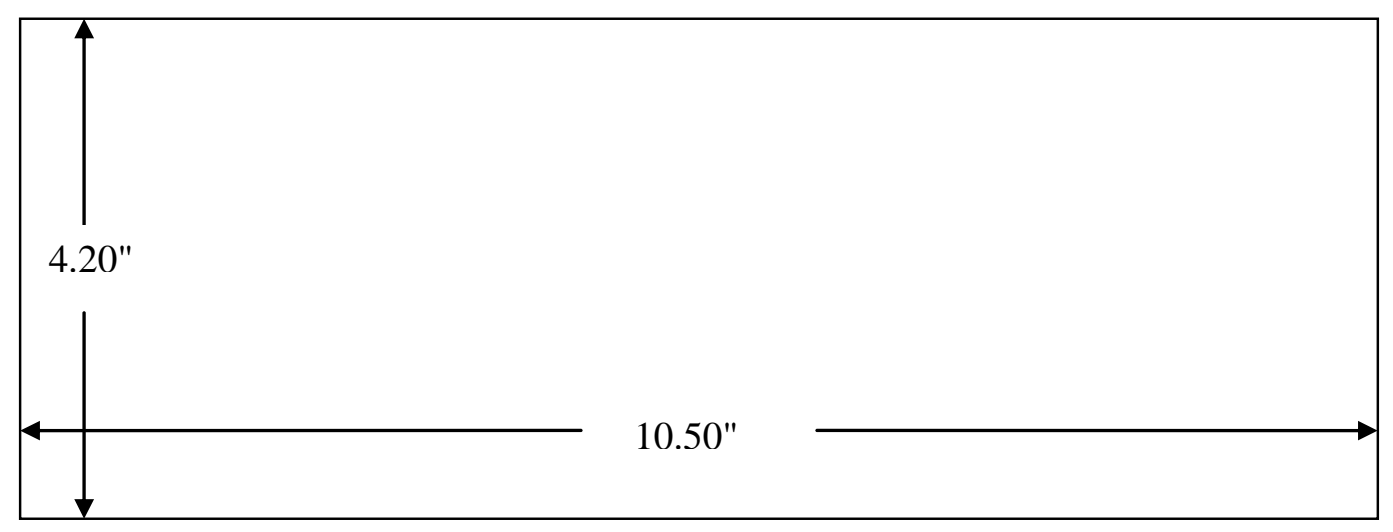

Figure 10: Specimens dimensions for S1-FM94 sandwich material system

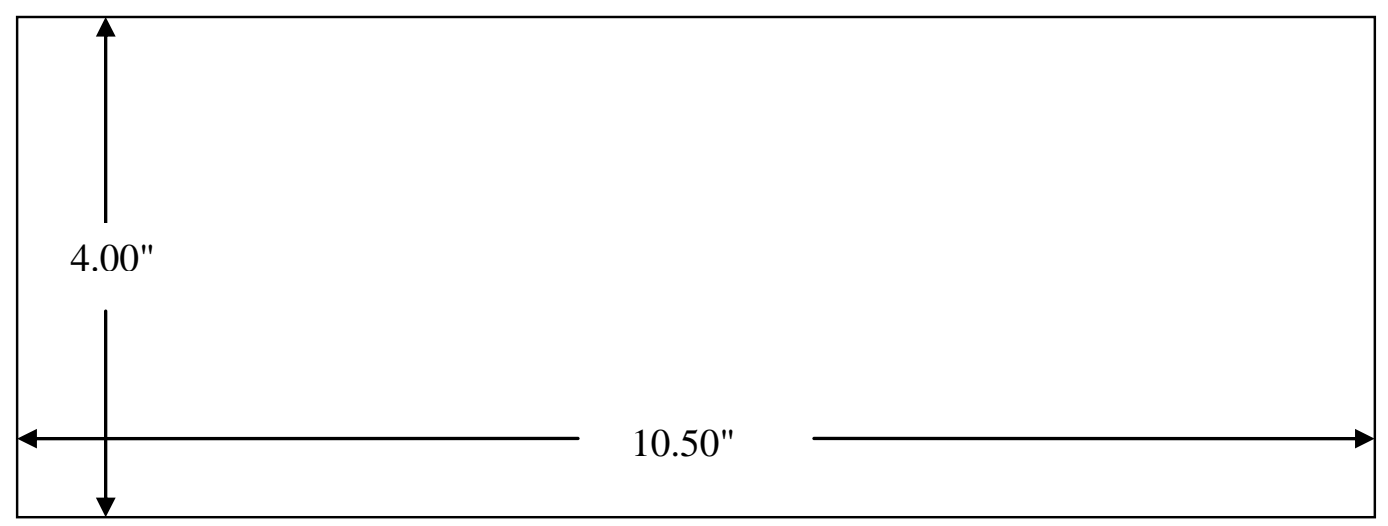

Figure 11: Specimens dimensions for S2-FM94, CRF and E2-H sandwich material systems

\subsection{Nomenclature}

The sandwich specimens after machining were labeled as illustrated in Table 4 before indentation tests. The indentation test was conducted as per the nomenclature shown in Table 4. In Table 4, a number followed by " $X$ " represents the indentation depth. The sandwich types are placed before the symbol " $\mathrm{X}$ ". 
TABLE 4

NOMENCLATURE OF INDENTED SPECIMENS

\begin{tabular}{|c|c|c|c|}
\hline $\begin{array}{c}\text { Sandwich } \\
\text { Type }\end{array}$ & Nomenclature & Configurations & Indentation Depth \\
\hline \multirow{4}{*}{ CRF } & \multirow{4}{*}{ CRFX0.15 } & Damage-No Repair & \multirow{4}{*}{$0.15^{\prime \prime}$} \\
\hline & & Damage-Repair & \\
\hline & & Destructive Inspection-No Repair & \\
\hline & & Destructive Inspection-Repair & \\
\hline \multirow{7}{*}{ S2-FM94 } & \multirow{4}{*}{ S2-FM94X0.10 } & Damage-No Repair & \multirow{4}{*}{$0.10^{\prime \prime}$} \\
\hline & & Damage-Repair & \\
\hline & & Destructive Inspection-No Repair & \\
\hline & & Destructive Inspection-Repair & \\
\hline & \multirow{3}{*}{ S2-FM94X0.15 } & Damage-No Repair & \multirow{3}{*}{$0.15^{\prime \prime}$} \\
\hline & & Damage-Repair & \\
\hline & & Destructive Inspection-No Repair & \\
\hline \multirow{8}{*}{ E2-H } & \multirow{4}{*}{ E2-HX0.10 } & Damage-No Repair & \multirow{4}{*}{$0.10^{\prime \prime}$} \\
\hline & & Damage-Repair & \\
\hline & & Destructive Inspection-No Repair & \\
\hline & & Destructive Inspection-Repair & \\
\hline & \multirow{4}{*}{ E2-HX0.15 } & Damage-No Repair & \multirow{4}{*}{$0.15^{\prime \prime}$} \\
\hline & & Damage-Repair & \\
\hline & & Destructive Inspection-No Repair & \\
\hline & & Destructive Inspection-Repair & \\
\hline
\end{tabular}

\subsection{Creation of Subsurface Core Damages}

The subsurface core damage states were created using low-velocity impact tests and quasi-static indentation tests. The low-velocity impact tests were used during the preliminary phase of the program. However, to ensure repeatability in creation of damage, quasi-static indentation tests were utilized in the remainder of the study. The details of the damage creation processes are discussed in the following sections. 


\subsubsection{Impact Tests}

The rectangular specimens (S1-FM94) of dimensions 10.50 inches tall and 4.20 inches wide were impacted using a 1.0" diameter impactor with impact energy of 40lb-in, that produced subsurface core damage underneath the facesheets. The impact testing was conducted at National Institute for Aviation Research (NIAR) using the DYNATUP instrumented impact testing machine. The impact tests were conducted at the geometric center (planar) of one of the facesheet with the other facesheet supported on an aluminum platen. The $40 \mathrm{lb}$-in energy level was arrived at by trial and error to achieve subsurface core damage states.

\subsubsection{Static Indentation Tests}

Before proceeding with an evaluation of in-plane compressive characteristics of impact damaged sandwich specimens, a preliminary impact damage characterization was necessary. To create subsurface core damage underneath the facesheet, the sandwich (CRF, S2-FM94 and E2H) specimens were indented using a semi-hemispherical steel impactor of 3.0" diameter at quasistatic rate of 0.05 in/min on a 5kip MTS 810 servo-hydraulic testing machine as shown in Figure 12 and Figure 13.

The sandwich specimens were subjected to in-plane compressive loading in a displacement controlled mode. Indentation depths of 0.10 " and 0.15 " were used on sandwich specimens as described by the test matrix in Table 3. An initial contact between the specimen surface and the indentor is established by moving the actuator using manual controls. The indentation loading is then performed at a constant indentation rate of $0.05 \mathrm{in} / \mathrm{min}$ after compressing to either, $0.10 "$ or $0.15 "$, the actuator unloads back to original position. E2-H and S2-FM94 sandwich specimens were indented to both 0.10 " and 0.15 " depths whereas CRF 
sandwich specimens were subjected to an indentation depth of 0.15". Figure 13 shows the CRF sandwich panel undergoing indentation testing.

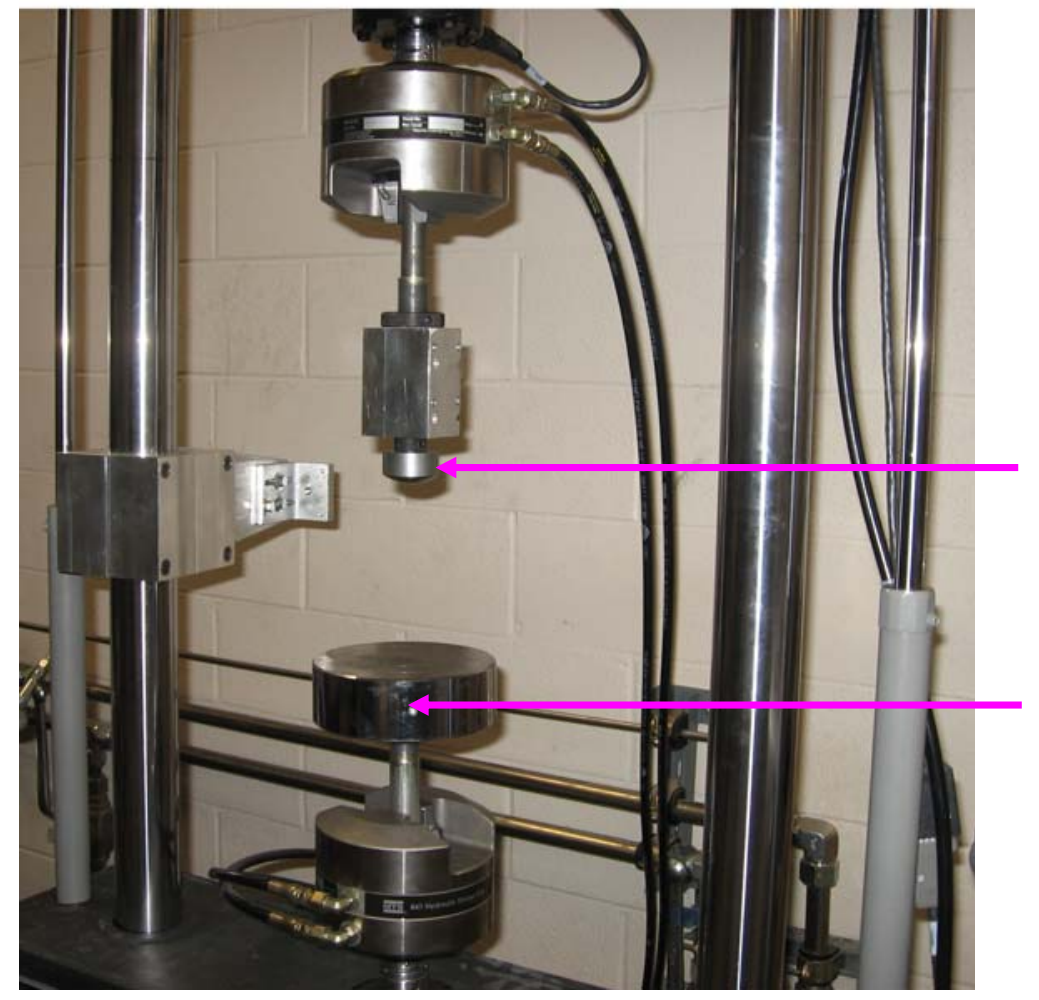

Hemispherical Steel Ball Indenter

Figure 12: Test Setup for Indentation Test

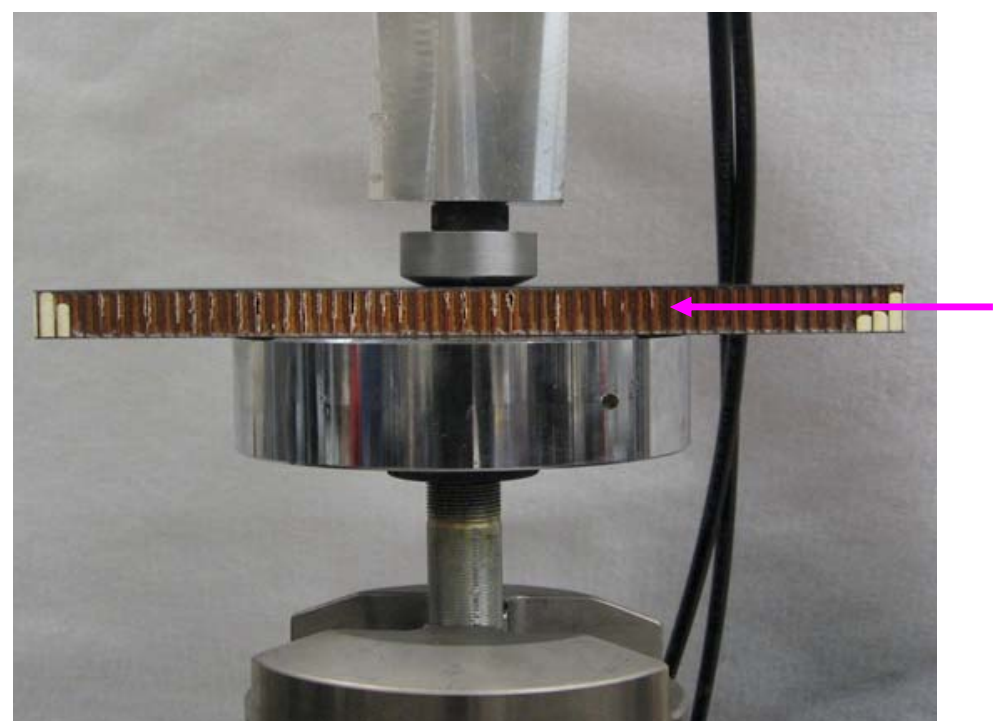

CRF Sandwich Panel

Figure 13: CRF Sandwich Specimen Indented at $0.05 \mathrm{in} / \mathrm{min}$ 


\subsection{Damage Characterization}

The damage induced in the sandwich panels were characterized using destructive and non-destructive methods. The geometric damage states were characterized in terms of the residual dent depth while the material damage states was characterized in terms of planar damage size and depth of core damage. The details of the destructive and non-destructive characterization are discussed in the following paragraphs.

\subsubsection{Nondestructive Evaluation of Damage}

Through Transmission Ultrasonic C-Scan (TTU) was used as the nondestructive inspection technique to obtain the planar damage area. The planar damage area represents the cumulative damage across the thickness. However, for sandwich panels, in the absence of facesheet damage, it is essentially the planar extent of the damaged in the core. The typical Cscan map obtained for a sandwich specimen is illustrated in Figure 14. The small grey colored circle like shape on the top left of Figure 14 represents the reference area which is equal to $0.1964 \mathrm{in}^{2}$. This small circle is the C-scan associated with a foam disc of known size bonded to the surface of the specimen for calibration purposes. The big grey colored almost circular shape represents the damage area due to indentation testing.

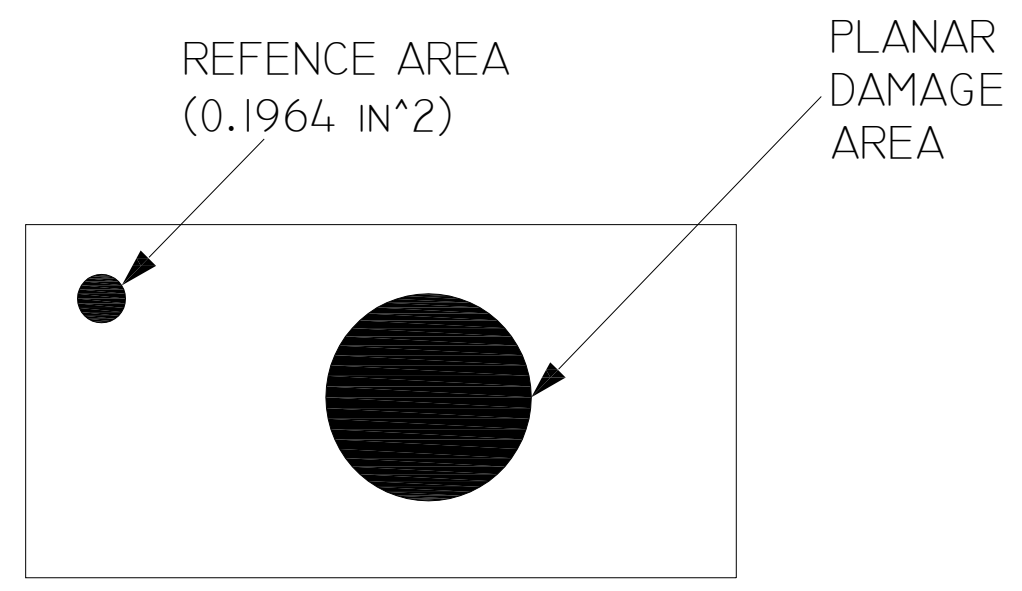

Figure 14: TTU C-scan planar damage area of indented sandwich panel before repair 
The residual indentation depth profile and the maximum residual dent depth were also used as a non-destructive method for characterizing damage in sandwich panels. The dent depths find applications in field inspections of sandwich structures. Further, the dent depth profile plays a crucial role in the modeling of the compression response of damaged sandwich panels. The dent depths were measured using digital dial gage. Two sets of readings along the longitudinal and lateral axes of the specimen geometry were noted down and the maximum residual indentation depth was tabulated. The pin of the digital dial gage was set to zero at far field (close to the edge of the specimen) from the center of the impact and/or indentation as the extreme points on the longitudinal and lateral axes of the damaged specimens are assumed to be flat or zero depth. The relative dent depth was measured using dial gage with respect to far field points. The profile of the points was then plotted to evaluate the true nature of the dimple and/or damage.

\subsubsection{Destructive Inspection of Damage}

The destructive sectioning of the one sandwich specimen from each indented sandwich configuration of all the three material systems was performed to visualize the exact nature of the damage and to correlate with the nondestructive damage evaluation techniques and the associated damage metrics. Evaluating the geometrical parameters (Figure 15) of the damaged honeycomb core is the main objective of destructive sectioning of the impact damaged sandwich specimens.

The sectioned sandwich specimens were further surface ground for better view of the section for destructive inspection and the cross sectional image was captured using a digital microscope. The subsurface core damage accompanied by a cavity resulting in cell wall buckling(s) was found to be dominant in all sectioned sandwich specimens. The dimensions of 
the damages are tabulated in Table 5. Figure 16 illustrates the presence of barely visible cracks and cell wall buckling in honeycomb core.

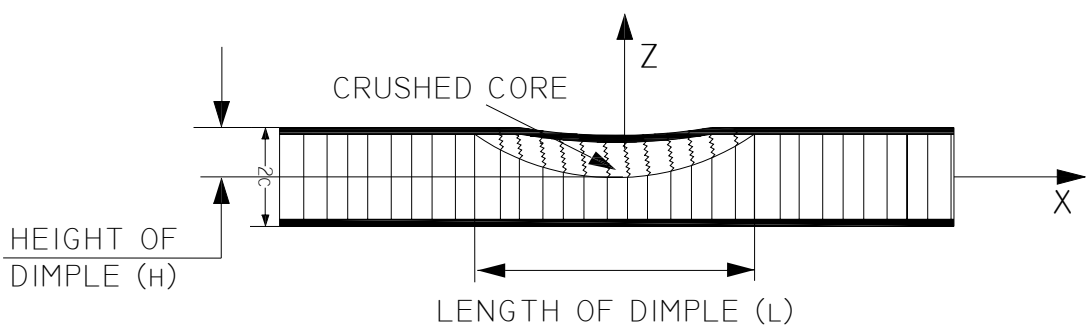

Figure 15: Dimensions of residual dent obtained by destructive sectioning of indented sandwich specimens

TABLE 5

SUMMARY OF DESTRUCTIVE INSPECTIONS OF DAMAGED SPECIMENS

\begin{tabular}{|c|c|c|c|c|}
\hline \multirow{2}{*}{ Specimen ID } & \multirow{2}{*}{ Configurations } & \multirow{2}{*}{$\begin{array}{c}\text { Indentation } \\
\text { Depth (in) }\end{array}$} & \multicolumn{2}{|c|}{ Cross Sectional Dimensions of Damage (in) } \\
\hline & & & Length (L) & Height (H) \\
\hline CRF-10 & \multirow{5}{*}{$\begin{array}{l}\text { Destructive } \\
\text { Inspection-No } \\
\text { Repair }\end{array}$} & 0.150 & 1.843 & 0.164 \\
\hline S2-FM94-16 & & 0.100 & 1.403 & 0.151 \\
\hline S2-FM94-18 & & 0.150 & 1.994 & 0.150 \\
\hline E2-H-16 & & 0.100 & 1.507 & 0.153 \\
\hline E2-H-18 & & 0.150 & 1.924 & 0.151 \\
\hline
\end{tabular}

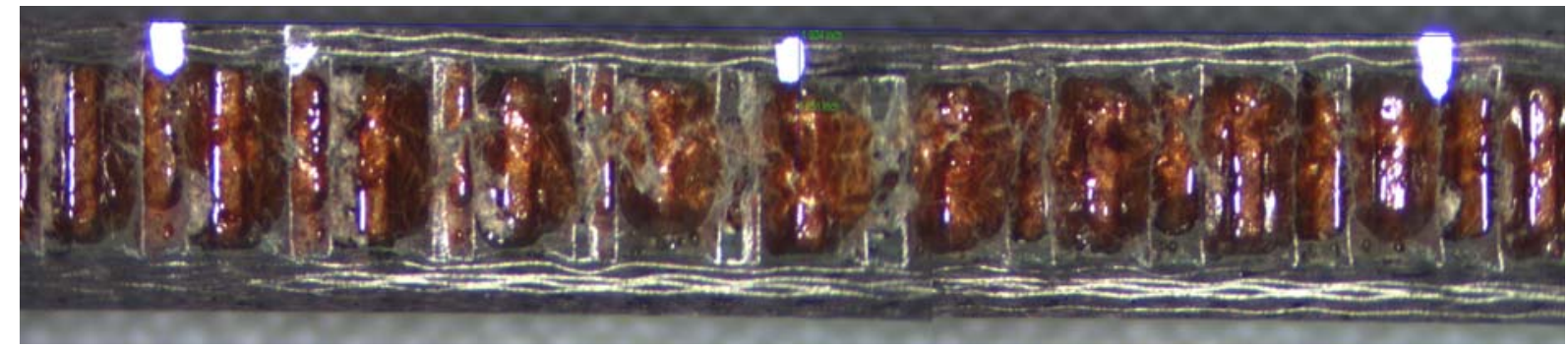

Figure 16: Cross sectional view of repaired E2-H-18

\subsection{Nondestructive Inspection of Repaired Specimens}

TTU C-Scan was used as nondestructive inspection technique to obtain the planar damage area of the repaired specimens before they were sectioned for destructive purposes. The damage area in this case is reduced compared to the NDI results of the damaged and unrepaired 
specimens. The resin filled cells at the edge and within the damage region appear as light-colored circles. The resin helps transmit the ultrasonic signal across the thickness of the sandwich panel and thus appears as an undamaged region as shown below.

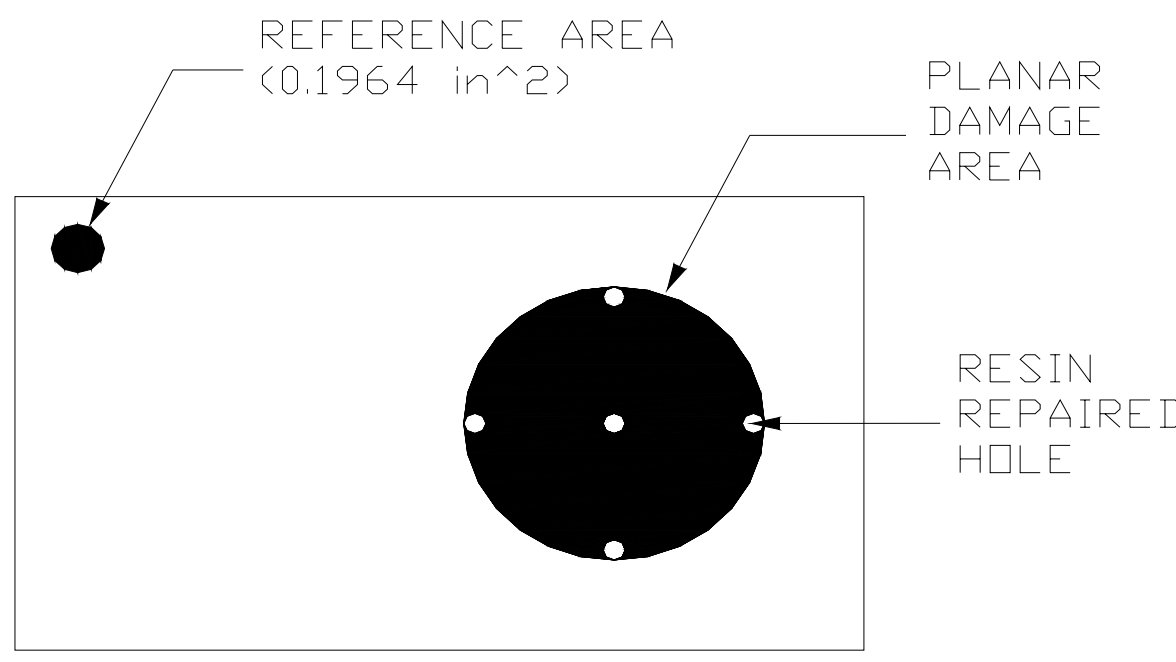

Figure 17: TTU C-scan planar damage area of indented sandwich panel after repair

\subsection{Destructive Inspection of Repaired Specimens}

The repaired sandwich specimens were sectioned after nondestructive inspection to characterize the subsurface core damage accompanied by resin filled cells. Figure 18 shows the sectional view of resin-repaired E2-H sandwich specimen that has damaged honeycomb core cell(s) filled with EPON resin mixture. The geometrical parameters of the destructive sectioning of the repaired specimens are summarized in Table 6.

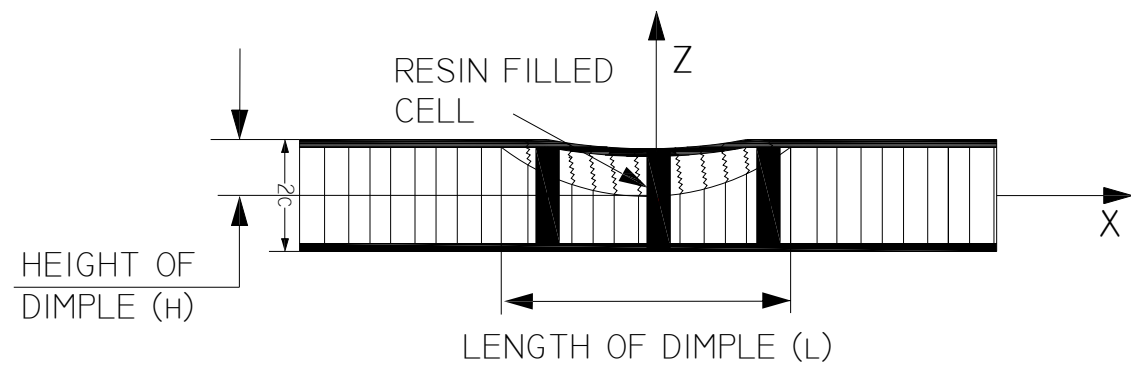

Figure 18: Dimensions of residual dent obtained by destructive sectioning of repaired sandwich specimens 


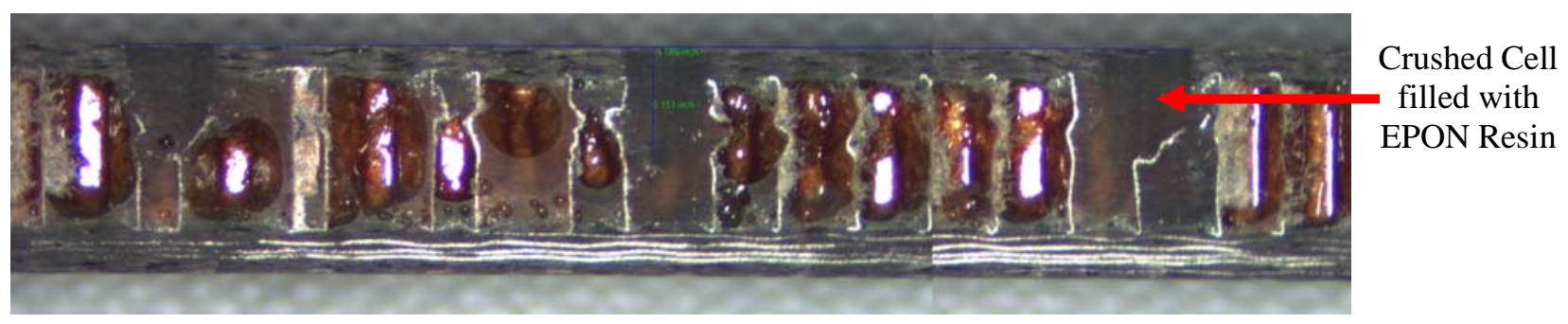

Figure 19: Cross sectional view of the repaired E2-H-17

TABLE 6

SUMMARY OF DESTRUCTIVE INSPECTIONS OF REPAIRED SPECIMENS

\begin{tabular}{|c|c|c|c|c|}
\hline \multirow{2}{*}{ Specimen ID } & \multirow{2}{*}{ Configurations } & \multirow{2}{*}{$\begin{array}{c}\text { Indentation } \\
\text { Depth (in) }\end{array}$} & \multicolumn{2}{|c|}{ Cross Sectional Dimensions of Damage } \\
\hline & & & Length (L) & Height (H) \\
\hline CRF-11 & \multirow{5}{*}{$\begin{array}{c}\text { Destructive } \\
\text { Inspection-Repair }\end{array}$} & 0.150 & 2.048" & $0.166 "$ \\
\hline S2-FM94-17 & & 0.100 & $1.510^{\prime \prime}$ & $0.150 "$ \\
\hline S2-FM94-19 & & 0.150 & $2.070 "$ & $0.150 "$ \\
\hline E2-H-17 & & 0.100 & $1.5696 "$ & $0.151 "$ \\
\hline E2-H-19 & & 0.150 & $2.040 "$ & $0.151 "$ \\
\hline
\end{tabular}

\subsection{Compression after Impact Testing}

All the sandwich specimens were tested under quasi-static edgewise compressive loading to obtain the residual strengths of undamaged, damaged and repaired specimens. The quasi-static in-plane compression tests were conducted on MTS 810 Servo-Hydraulic testing machine at National Institute for Aviation Research (NIAR). The tests were conducted under displacement control mode at a constant actuator displacement rate of $0.05 \mathrm{in} / \mathrm{min}$. The test apparatus used in the study is illustrated in Figures 20 and 21. 


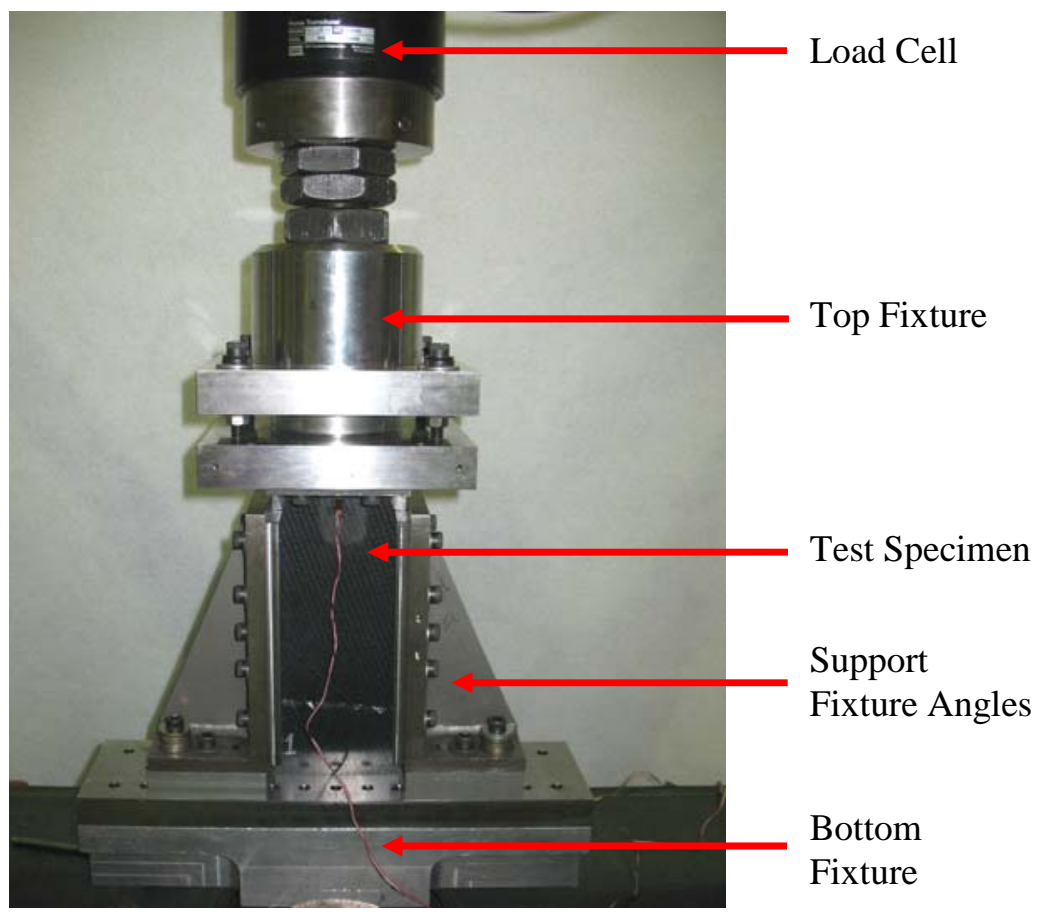

Figure 20: Schematic of compressive residual strength support fixture with specimen in place

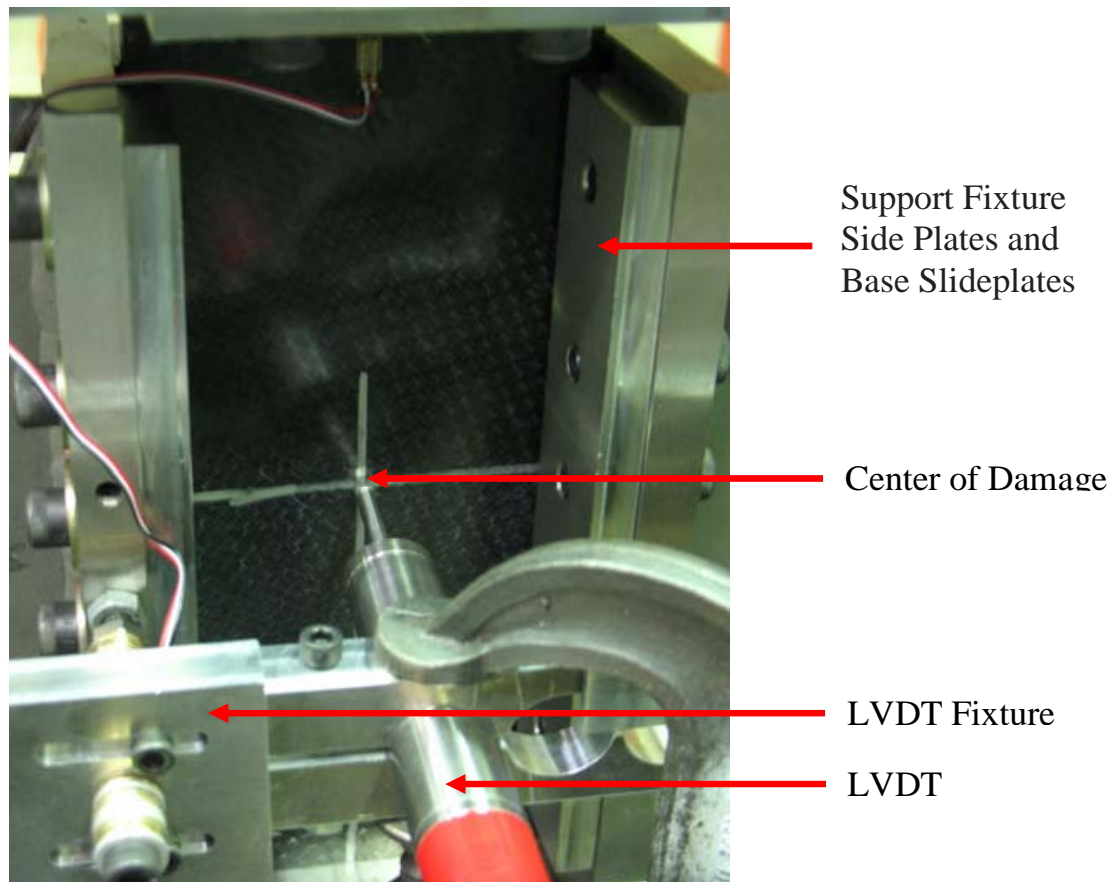

Figure 21: LVDT fixture and LVDT placed at the center of the damage region 
The boundary conditions imposed on the test specimen is illustrated in Figure 22. The specimen was simply-supported along its vertical edges by knife-edge supports which were fastened to the support angles. The bottom platen was connected to the actuator while the adjustable top platen was fastened to the load cell, which in turn was fastened to the fixed crosshead of the testing machine. The adjustable platen is used to ensure that load is evenly distributed across the width of the panel and between the two facesheets. The loading edges (top and bottom) were subjected to clamped edge conditions as shown in Figure 22.

The out-of-plane displacement in the sandwich panel, especially at the center of the damage region, which indicates the activity of the damage under in-plane compressive loading, was monitored using a LVDT (DCTH500A [15]). The LVDT mounted on the fixture is illustrated in Figure 21. Since the test specimen undergoes in-plane deformation, the location of the center of the damage region displaces in the loading direction. In order to eliminate the relative displacement (along the loading direction) between the center of the damage region and the LVDT, the LVDT was mounted on a trammel mechanism. The trammel mechanism displaces the LVDT by an amount equal to one-half the end-shortening of the specimen, resulting in negligible in-plane relative displacement between the specimen and the LVDT. Thus, the LVDT always measured the out-of-plane displacement at the center of the damage region.

Two back-to-back strain gages (Micro-Measurements CEA-06-250UN-120 [16]) were bonded to the specimens as shown in Figure 22. The strain gages served two purposes. The balancing of load distribution between the two facesheets was achieved by monitoring the strains in the two facesheets. A proof load of $25 \mathrm{lb}$ was applied to the specimen and the alignment platen was adjusted until the strain gage readings were within a tolerance. A tolerance of 50 microstrains was generally used in balancing the specimens. Upon satisfactorily balancing the 
load distribution, the specimen was unloaded. The test specimen was subsequently loaded monotonically to failure. The two strain gages were then used to monitor the far-filed strains during the tests and the strains at failure. An appreciable deviation between the two strain gage readings indicated global buckling in the test specimen. The test specimens were loaded until failure and the actuator displacement, load, LVDT and strain gage readings were continuously recorded. The data acquisition was accomplished using TestWorks computer program. The recorded data was subsequently analyzed to characterize the sandwich behavior under edgewise compressive loading.
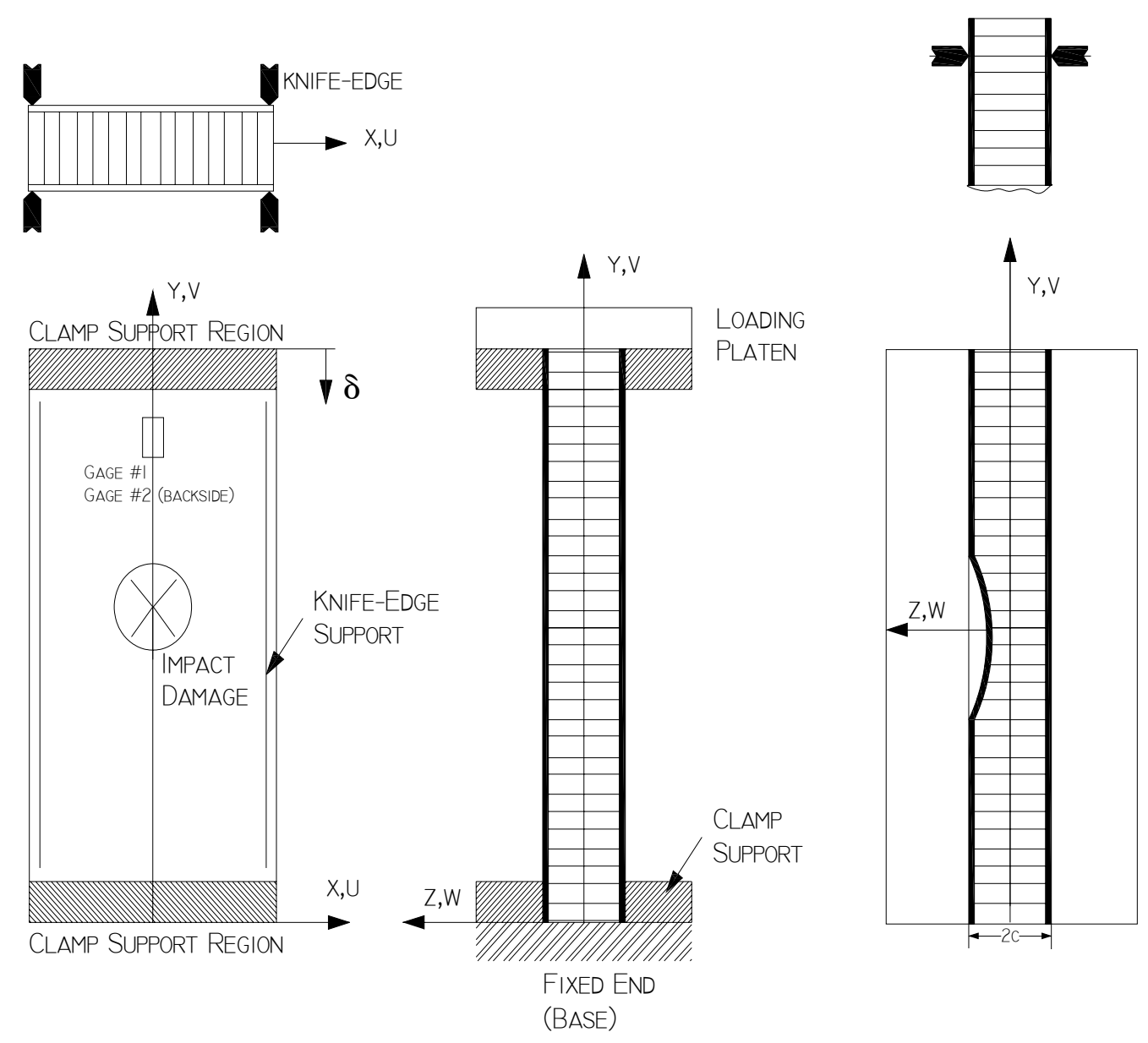

Figure 22: Boundary conditions and unconstrained local out-of-plane displacement along the knife-edges used in compression test [3] 


\section{CHAPTER 5}

\section{RESULTS AND DISCUSSIONS}

The experimental results from the damage creation and repair, their characterization using destructive and non-destructive methods and the effects of damage and repair processes on the in-plane compression responses of sandwich panels are discussed in this chapter.

\subsection{Characterization of Subsurface Damage}

The subsurface damage created using low-velocity impacts and static indentation tests were characterized using destructive and non-destructive methods described in the previous chapters. The damage in sandwich panels was characterized in terms of the TTU C-scan planar damage area, residual dent depth profiles and the maximum residual dent depth. A limited number of representative specimens were sectioned destructively to study the true nature of the core damage and the honeycomb cells filled with resin from the repair process.

The surface profiles of the impact or indentation damaged sandwich specimens were plotted as residual dent depth (in) along y-axis and the distance moved (in) along x-axis. The typical surface profiles of damaged sandwich specimens are plotted as shown in Figure 23 and the maximum residual indentation depths are tabulated in Table 7. It is evident that the surface

profiles of the damaged specimens are symmetric about x-axis indicating the uniformity of damage shapes on the specimens. 


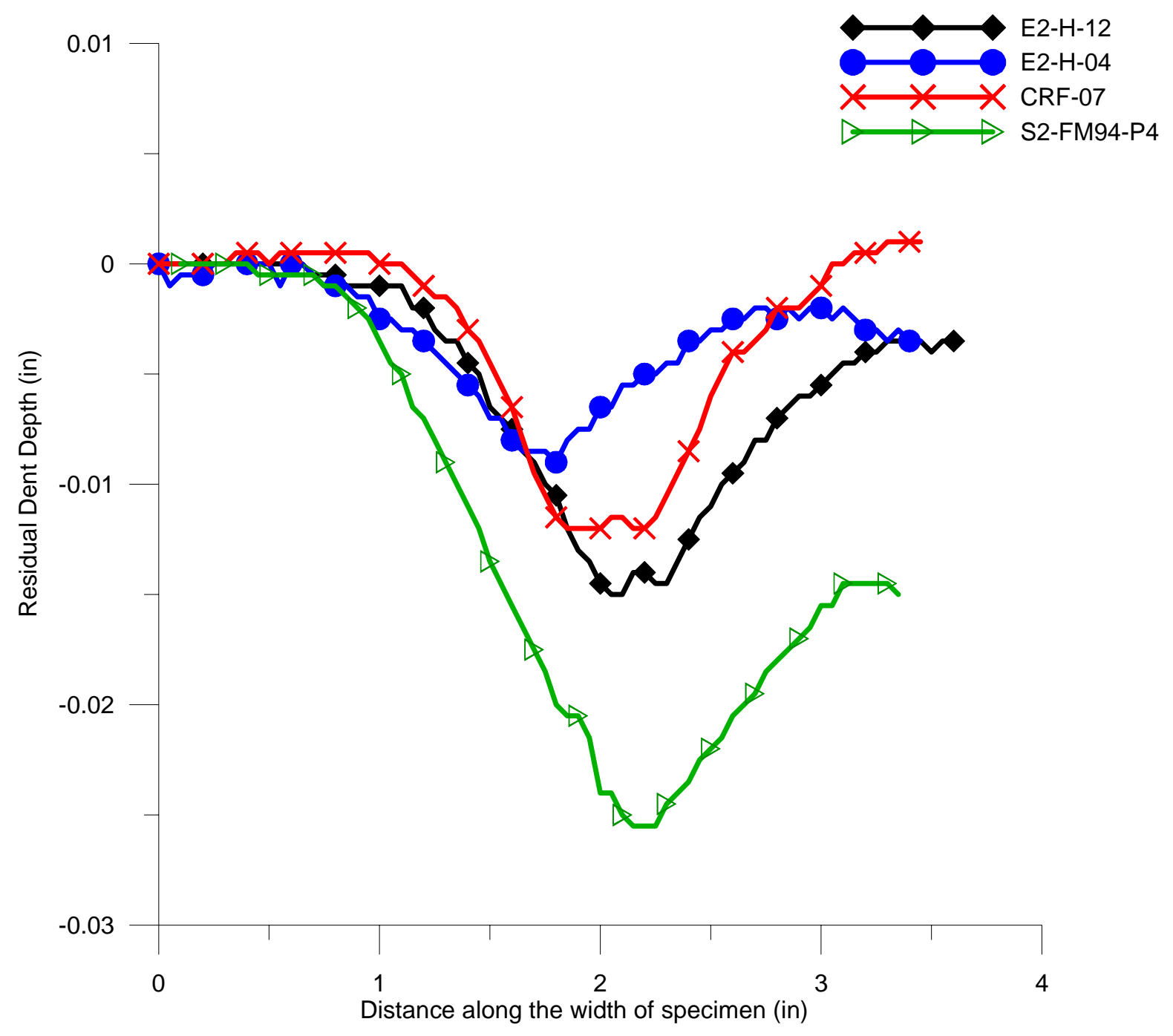

Figure 23: Surface profiles of indented sandwich specimens 
TABLE 7

SUMMARY OF RESIDUAL DENT MEASUREMENTS

\begin{tabular}{|c|c|c|c|}
\hline Specimen ID & Configurations & Indentation Depth & $\begin{array}{l}\text { Max. Residual } \\
\text { Dent Depth }\end{array}$ \\
\hline CRF-4 & \multirow{3}{*}{ Damage-No Repair } & \multirow{8}{*}{$0.15 "$} & $0.0205 "$ \\
\hline CRF-5 & & & $0.0265 "$ \\
\hline CRF-6 & & & $0.0155 "$ \\
\hline CRF-7 & \multirow{3}{*}{ Damage-Repair } & & $0.0250 "$ \\
\hline CRF-8 & & & $0.0145 "$ \\
\hline CRF-9 & & & $0.0150 "$ \\
\hline CRF-10 & Destructive Inspection-No Repair & & $0.0175 "$ \\
\hline CRF-11 & Destructive Inspection-Repair & & $0.0145 "$ \\
\hline S2-FM94-4 & \multirow{3}{*}{ Damage-No Repair } & \multirow{8}{*}{$0.10^{\prime \prime}$} & $0.0155 "$ \\
\hline S2-FM94-5 & & & $0.0195 "$ \\
\hline S2-FM94-6 & & & $0.0125 "$ \\
\hline S2-FM94-7 & \multirow{3}{*}{ Damage-Repair } & & $0.0120^{\prime \prime}$ \\
\hline S2-FM94-8 & & & $0.0125 "$ \\
\hline S2-FM94-9 & & & $0.0120 "$ \\
\hline S2-FM94-16 & Destructive Inspection-No Repair & & $0.0135 "$ \\
\hline S2-FM94-17 & Destructive Inspection-Repair & & $0.0045 "$ \\
\hline S2-FM94-10 & \multirow{3}{*}{ Damage-No Repair } & \multirow{7}{*}{$0.15^{\prime \prime}$} & $0.0255 "$ \\
\hline S2-FM94-11 & & & $0.0255^{\prime \prime}$ \\
\hline S2-FM94-12 & & & $0.0250 "$ \\
\hline S2-FM94-13 & \multirow{3}{*}{ Damage-Repair } & & $0.0225 "$ \\
\hline S2-FM94-14 & & & $0.0240 "$ \\
\hline S2-FM94-15 & & & $0.0260 "$ \\
\hline S2-FM94-18 & Destructive Inspection-No Repair & & 0.0185 \\
\hline E2-H-4 & \multirow{3}{*}{ Damage-No Repair } & \multirow{8}{*}{$0.10^{\prime \prime}$} & $0.0090 "$ \\
\hline E2-H-5 & & & $0.0090 "$ \\
\hline E2-H-6 & & & $0.0095 "$ \\
\hline E2-H-7 & \multirow{3}{*}{ Damage-Repair } & & $0.0125 "$ \\
\hline E2-H-8 & & & $0.0095 "$ \\
\hline E2-H-9 & & & $0.0140 "$ \\
\hline E2-H-16 & Destructive Inspection-No Repair & & $0.0110^{\prime \prime}$ \\
\hline E2-H-17 & Destructive Inspection-Repair & & $0.0110^{\prime \prime}$ \\
\hline E2-H-10a & \multirow{3}{*}{ Damage-No Repair } & \multirow{8}{*}{$0.15 "$} & $0.0175 "$ \\
\hline E2-H-11 & & & $0.0165 "$ \\
\hline E2-H-12 & & & $0.0160 "$ \\
\hline E2-H-13 & \multirow{3}{*}{ Damage-Repair } & & $0.0155 "$ \\
\hline E2-H-14 & & & $0.0265 "$ \\
\hline E2-H-15 & & & $0.0160 "$ \\
\hline E2-H-18 & Destructive Inspection-No Repair & & $0.0190 "$ \\
\hline E2-H-19 & Destructive Inspection-Repair & & $0.0165 "$ \\
\hline
\end{tabular}


The TTU C-scan method was used to characterize the planar extent of damage in sandwich panels. The typical C-scan images of the damaged panels are shown in Figures 24 through 26. The damage regions were observed to be roughly circular in shape. The planar size of the damage region was quantified in terms of planar damage area using an image analysis program. The planar damage area for the different sandwich configurations are summarized in Table 8 . The planar damage areas are ranged between $1.5 \mathrm{in}^{2}$ to $3.5 \mathrm{in}^{2}$, with the area increasing with applied indentation depth.

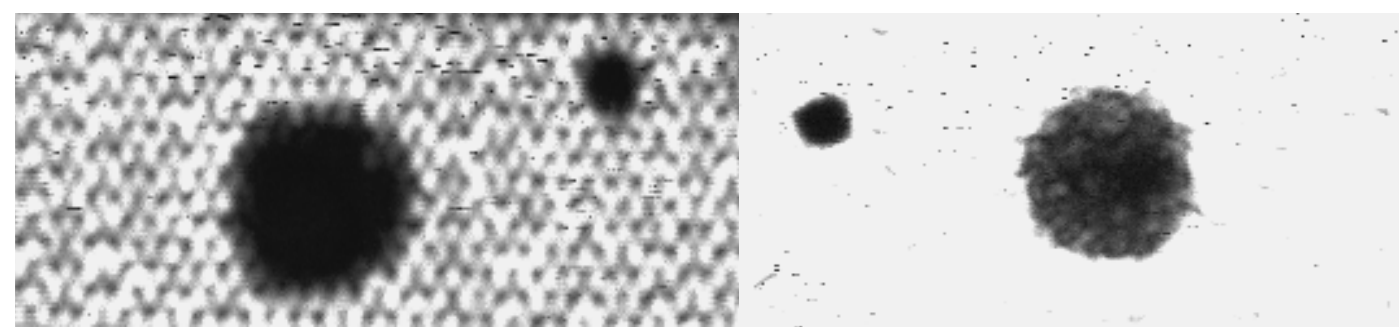

Figure 24: TTU C-scan image of CRF-4 (left) and S2-FM94-4 (right) panels with damage

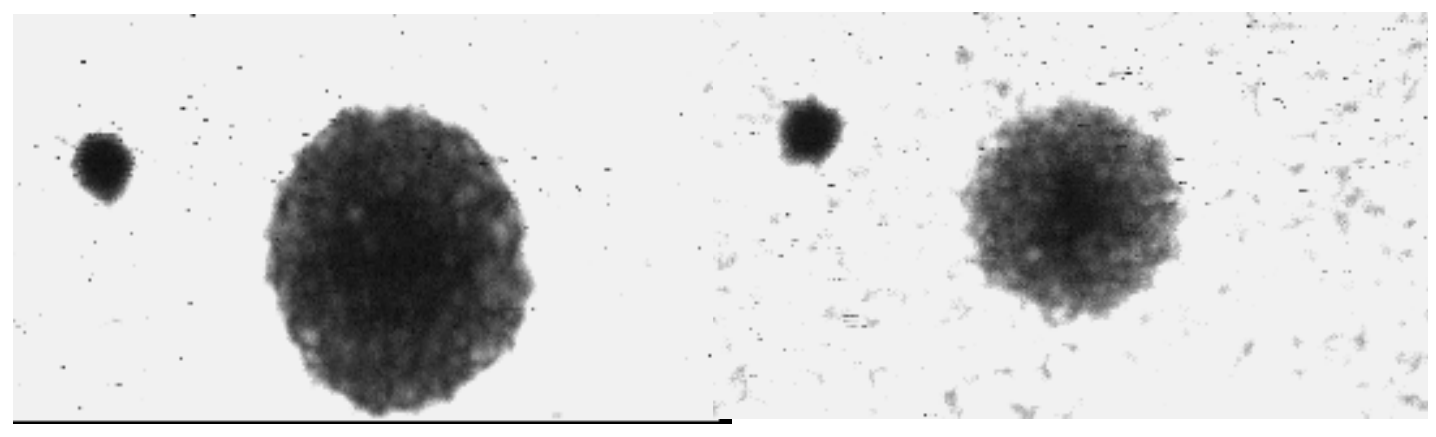

Figure 25: TTU C-scan image of S2-FM94-P10 (left) and E2-H-4 (right) panels with damage

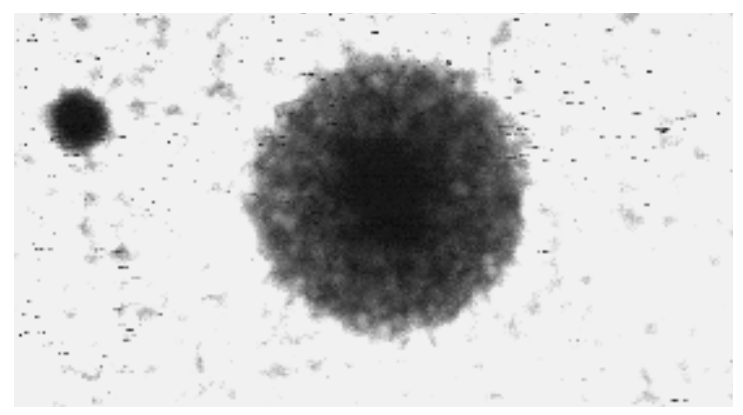

Figure 26: TTU C-scan image of E2-H-12 panel with damage 
TABLE 8

SUMMARY OF NONDESTRUCTIVE INSPECTIONS OF DAMAGED SPECIMENS

\begin{tabular}{|c|c|c|c|}
\hline Specimen ID & Configurations & $\begin{array}{c}\text { Indentation } \\
\text { Depth (in) }\end{array}$ & $\begin{array}{c}\text { Planar Damage } \\
\left.\text { Area (in }{ }^{2}\right)\end{array}$ \\
\hline CRF-4 & \multirow{3}{*}{ Damage-No Repair } & \multirow{4}{*}{0.15} & 2.1672 \\
\hline CRF-5 & & & 1.8392 \\
\hline CRF-6 & & & 1.9736 \\
\hline CRF-10 & Destructive Inspection-No Repair & & 2.0944 \\
\hline S2-FM94-4 & \multirow{3}{*}{ Damage-No Repair } & \multirow{4}{*}{0.10} & 1.7564 \\
\hline S2-FM94-5 & & & 1.7676 \\
\hline S2-FM94-6 & & & 1.8552 \\
\hline S2-FM94-16 & Destructive Inspection-No Repair & & 1.6296 \\
\hline S2-FM94-10 & \multirow{3}{*}{ Damage-No Repair } & \multirow{4}{*}{0.15} & 3.4140 \\
\hline S2-FM94-11 & & & 3.5020 \\
\hline S2-FM94-12 & & & 3.4912 \\
\hline S2-FM94-18 & Destructive Inspection-No Repair & & 3.3292 \\
\hline E2-H-4 & \multirow{3}{*}{ Damage-No Repair } & \multirow{4}{*}{0.10} & 1.9748 \\
\hline E2-H-5 & & & 1.8928 \\
\hline E2-H-6 & & & 1.9312 \\
\hline E2-H-16 & Destructive Inspection-No Repair & & 1.9000 \\
\hline E2-H-10a & \multirow{3}{*}{ Damage-No Repair } & \multirow{4}{*}{0.15} & 3.1220 \\
\hline E2-H-11 & & & 3.2524 \\
\hline E2-H-12 & & & 3.4968 \\
\hline E2-H-18 & Destructive Inspection-No Repair & & 3.1632 \\
\hline
\end{tabular}

A limited number of sandwich specimens (one specimen per combination of sandwich configuration and indentation depth level) were sectioned destructively across the damage region. The sectioning was conducted to study the true nature of the subsurface damage and its correlation with the planar damage size. The cross-sectional views of damaged sandwich specimens are illustrated in Figures 27 through 31. The figures indicate cell wall buckling and cell wall fractures/tearing in all the sandwich configurations. In sandwich specimens with thin core (E2-H-XX), the cell wall damage was spread across the thickness of the core. However, in specimens with thicker core, the core damage was localized to regions close to the indented facesheets. 


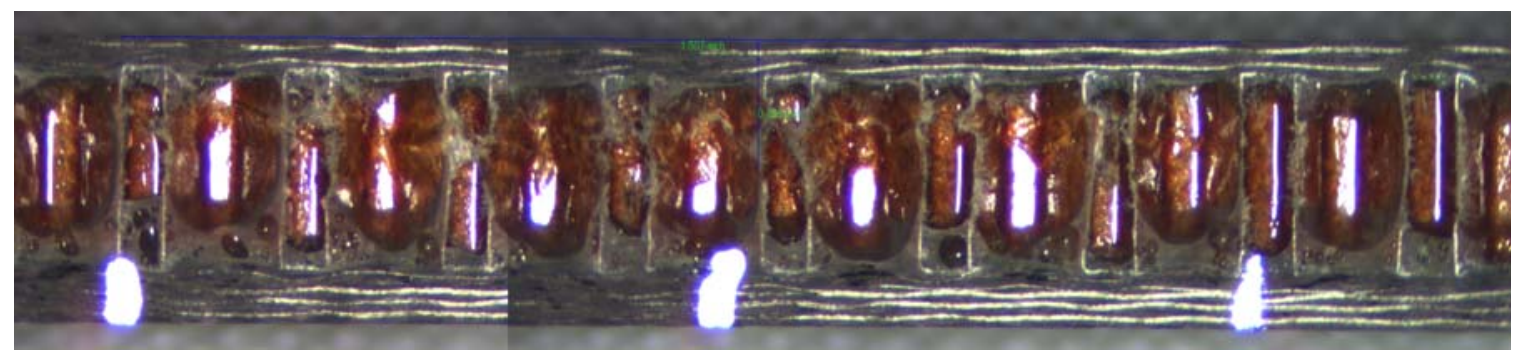

Figure 27: Destructive sectioning of E2-H-16

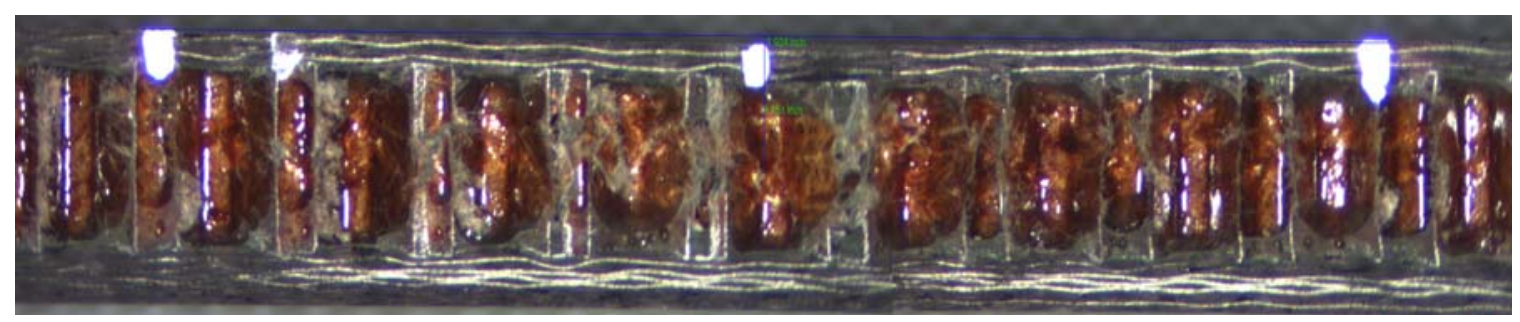

Figure 28: Destructive sectioning of E2-H-18

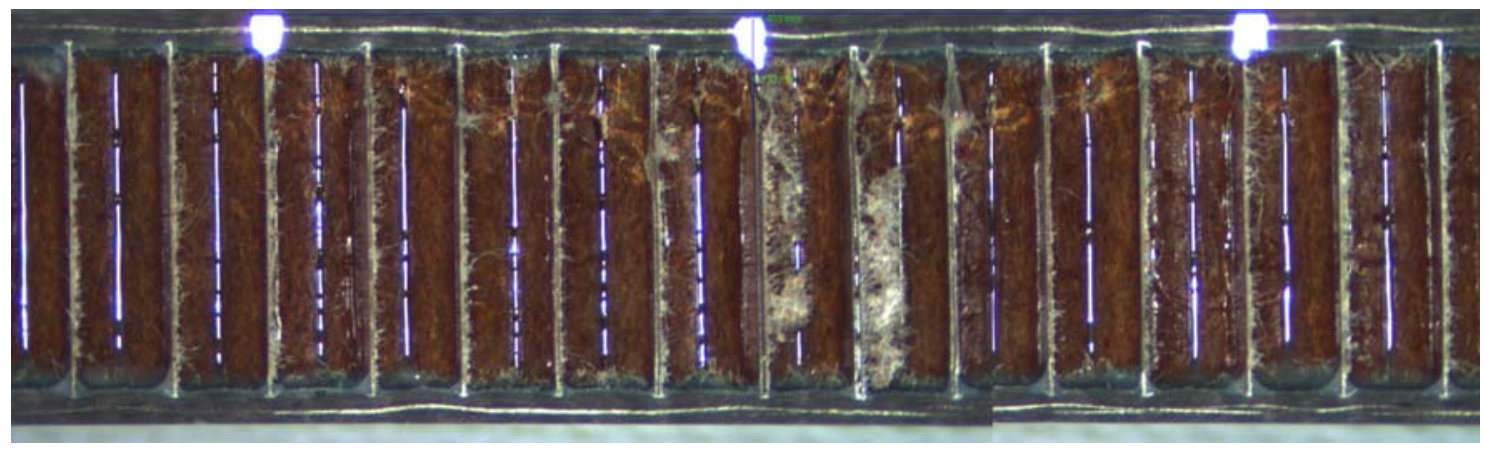

Figure 29: Destructive sectioning of S2-FM94-P16

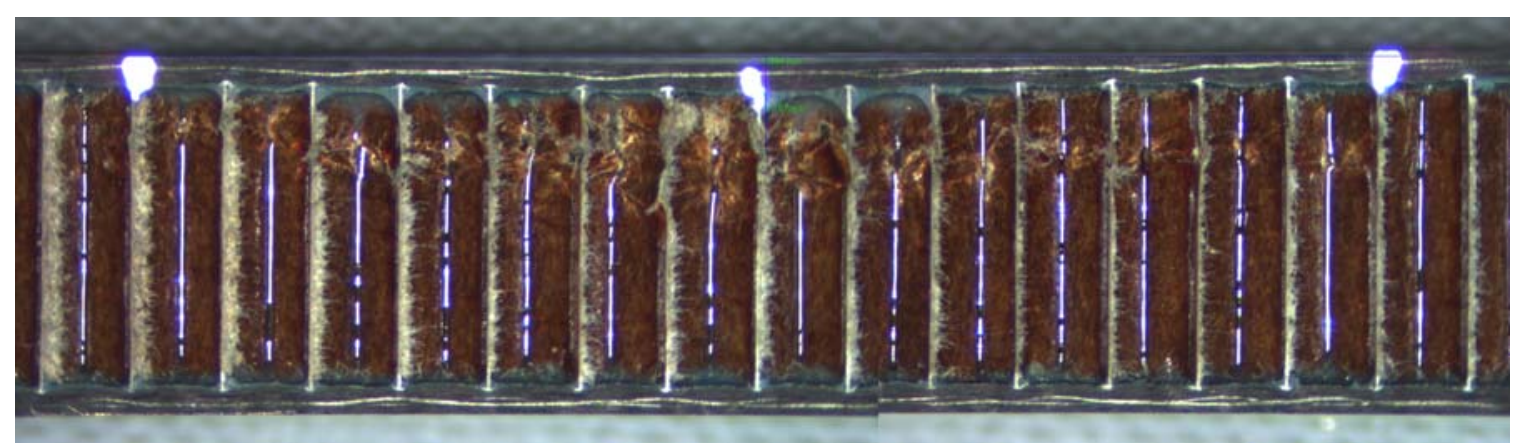

Figure 30: Destructive sectioning of S2-FM94-P18 


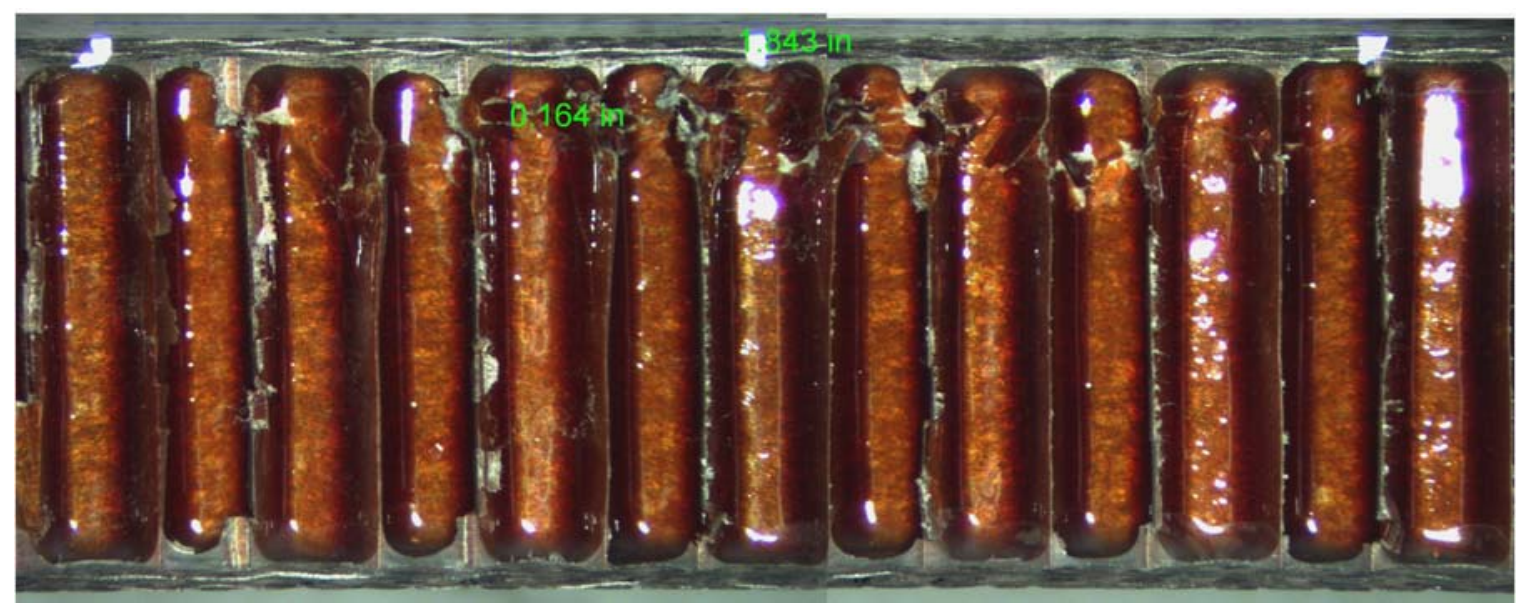

Figure 31: Destructive sectioning of CRF-10

\subsection{Characterization of Repair}

The damaged sandwich panels subjected to non-destructive inspection were subsequently repaired using the cell-fill method described in the previous chapters. The repaired panels were subsequently subjected to the TTU C-scan inspection to obtain a measure of the repaired region and a limited number of specimens were sectioned destructively to visualize the resin filling in the repaired panels.

The typical TTU C-scans of repaired sandwich panels are compared with their respective C-scans in the unrepaired state in Figures 32 through 39. The resin repairs appear as white circular regions within the darker damage region in the C-scan image. The comparison of planar damage areas of three sandwich material systems are summarized in Table 9. The planar damage areas corresponding to repaired specimens were observed to be slightly lower than the respective damage area prior to repair. This decrease is due to the ability of resin filled cells to transmit the ultrasonic signals across the thickness of the core. 
TABLE 9

SUMMARY OF NONDESTRUCTIVE INSPECTIONS OF REPAIRED SPECIMENS

\begin{tabular}{|c|c|c|c|}
\hline Specimen ID & Configurations & $\begin{array}{l}\text { Indentation Depth } \\
\text { (in) }\end{array}$ & $\begin{array}{c}\text { Planar Damage } \\
\text { Area }\left(\text { in }^{2}\right)\end{array}$ \\
\hline CRF-7 & \multirow{3}{*}{ Damage-Repair } & \multirow{4}{*}{ (ii) } & 2.4576 \\
\hline CRF-8 & & & 2.7344 \\
\hline CRF-9 & & & 2.3236 \\
\hline CRF-11 & Destructive Inspection-Repair & & 2.6176 \\
\hline S2-FM94-7 & \multirow{3}{*}{ Damage-Repair } & \multirow{4}{*}{0.10} & 1.9148 \\
\hline S2-FM94-8 & & & 1.7616 \\
\hline S2-FM94-9 & & & 1.6064 \\
\hline S2-FM94-17 & Destructive Inspection-Repair & & 1.0344 \\
\hline S2-FM94-13 & \multirow{3}{*}{ Damage-Repair } & \multirow{4}{*}{0.15} & 3.5840 \\
\hline S2-FM94-14 & & & 3.3492 \\
\hline S2-FM94-15 & & & 3.4004 \\
\hline S2-FM94-19 & Destructive Inspection-Repair & & 3.1776 \\
\hline E2-H-7 & \multirow{3}{*}{ Damage-Repair } & \multirow{4}{*}{0.10} & 1.6540 \\
\hline E2-H-8 & & & 1.4384 \\
\hline E2-H-9 & & & 1.4228 \\
\hline E2-H-17 & Destructive Inspection-Repair & & 1.5648 \\
\hline E2-H-13 & \multirow{3}{*}{ Damage-Repair } & \multirow{4}{*}{0.15} & 2.7292 \\
\hline E2-H-14 & & & 2.4092 \\
\hline E2-H-15 & & & 2.5780 \\
\hline E2-H-19 & Destructive Inspection-Repair & & 2.7228 \\
\hline
\end{tabular}

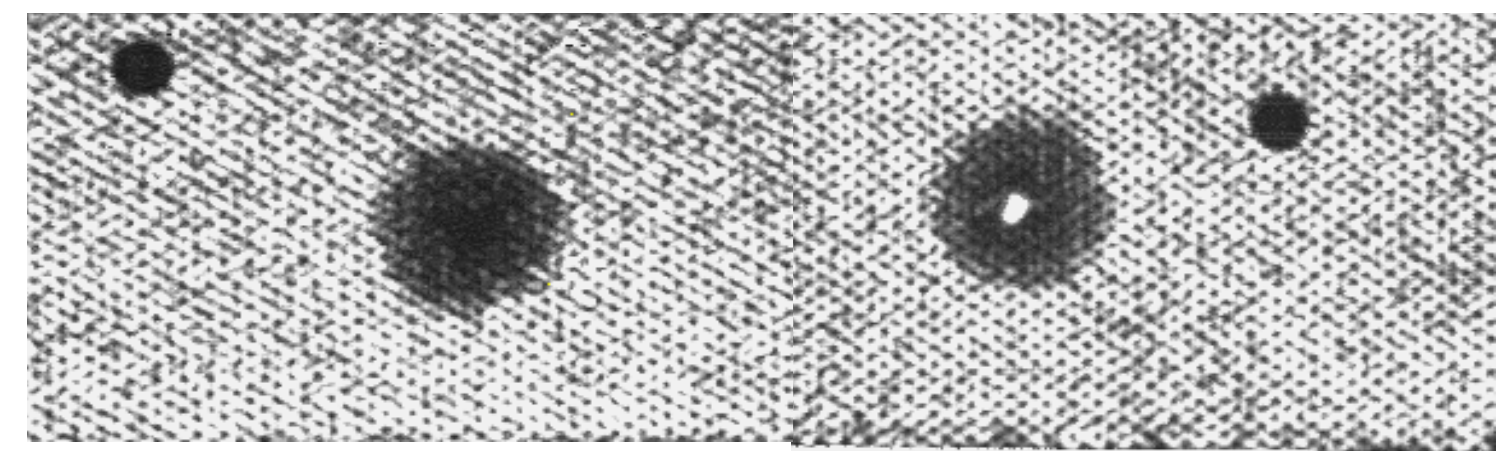

Figure 32: TTU C-scan image of S1-FM94-P9-2 with (right) and without (left) 1-Hole Repair 


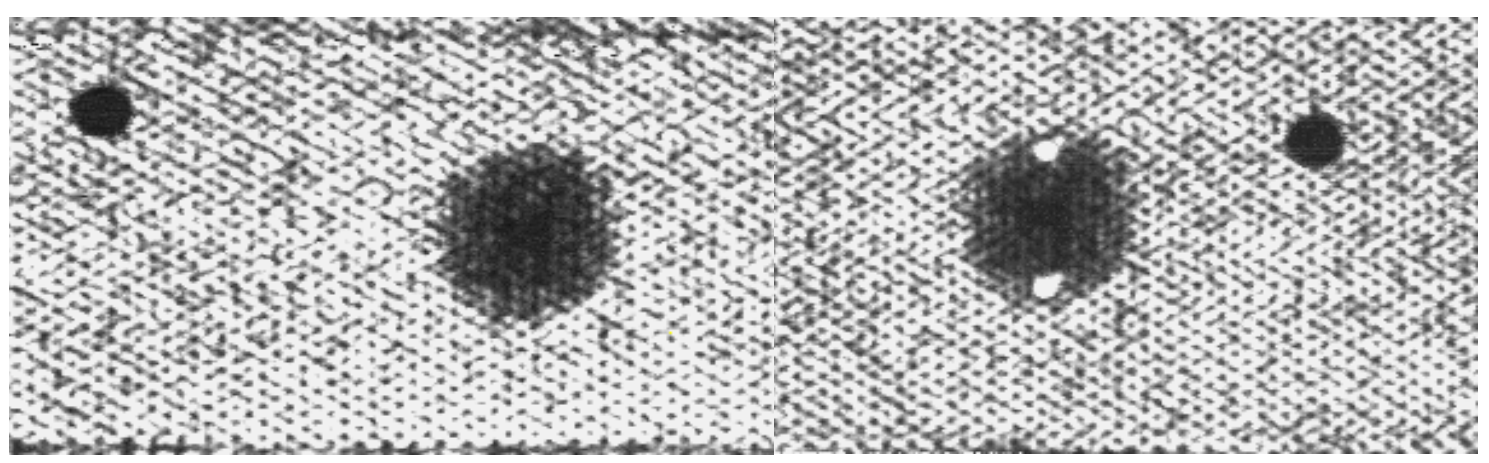

Figure 33: TTU C-scan image of S1-FM94-P11-3 with (right) and without (left) 2-Hole Repair

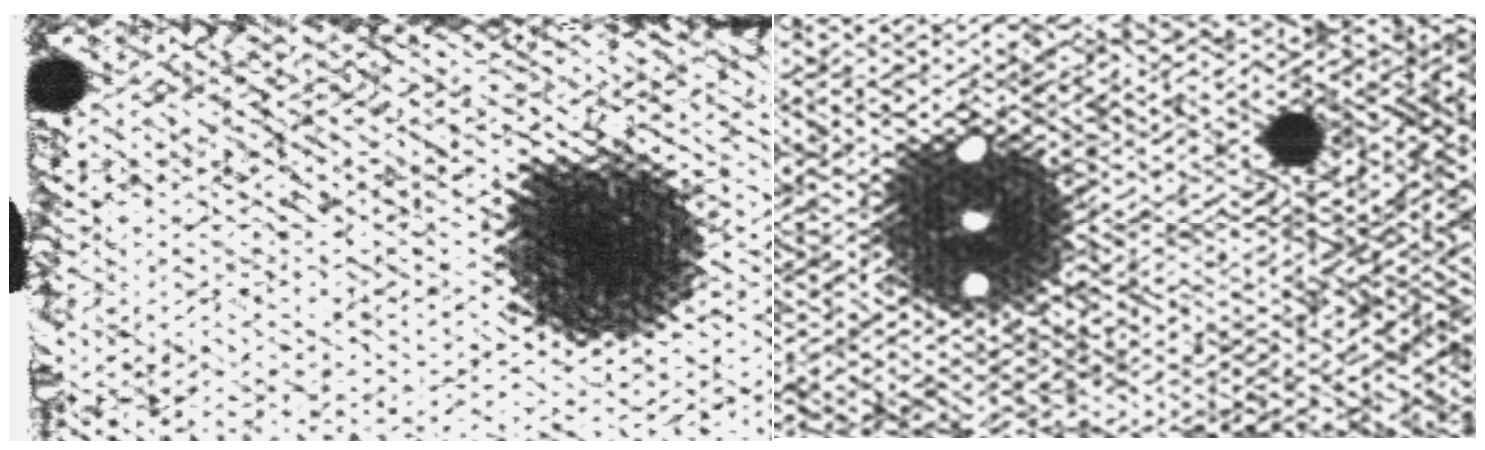

Figure 34: TTU C-scan image of S1-FM94-P10-3 with (right) and without (left) 3-Hole Repair

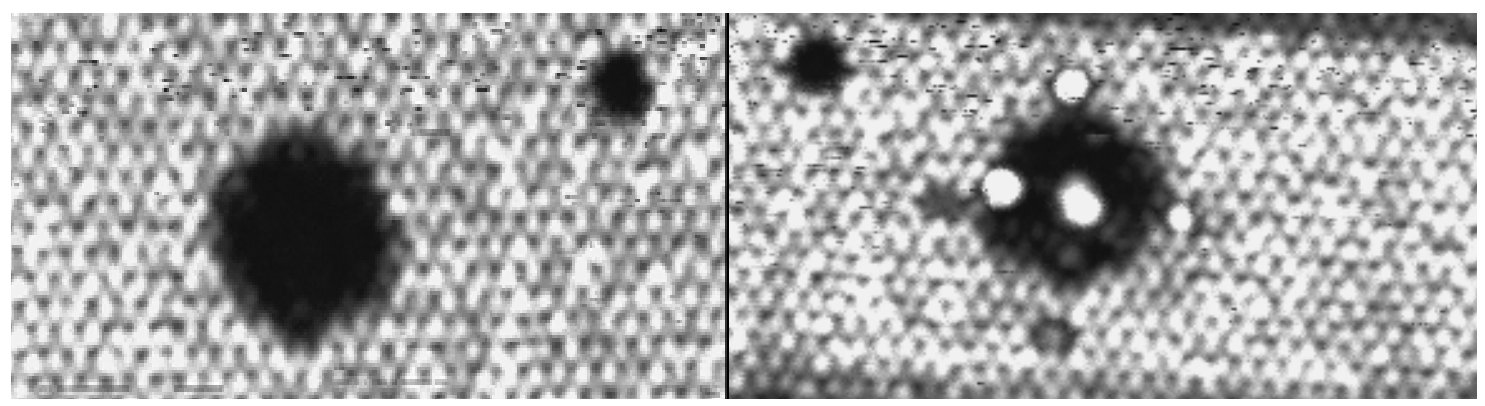

Figure 35: TTU C-scan image of CRF-09 with (right) and without (left) 3-Hole Repair

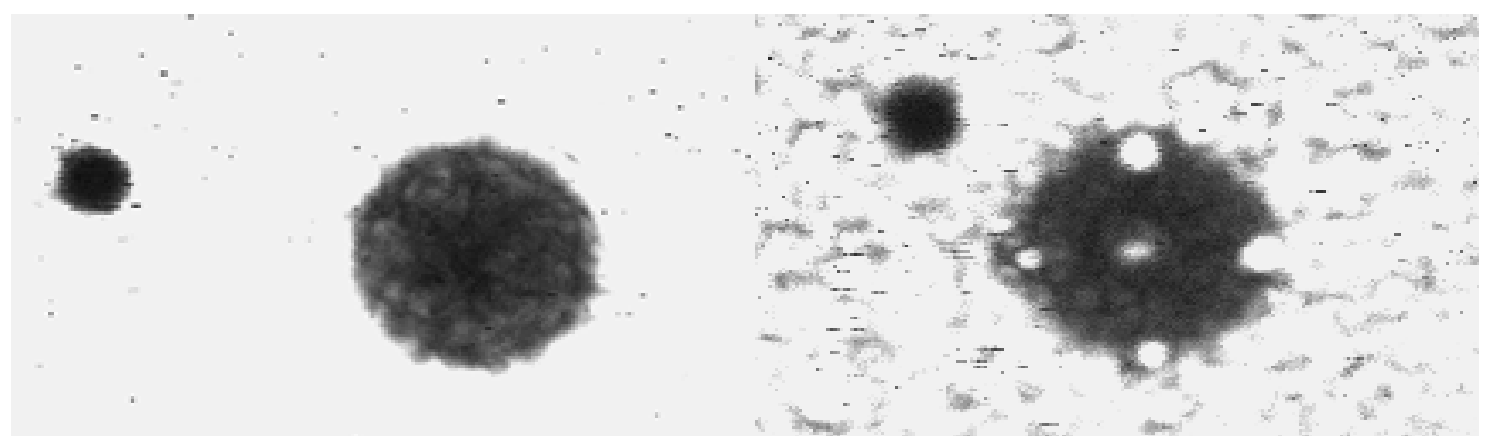

Figure 36: TTU C-scan image of S2-FM94-8 with (right) and without (left) 5-Hole Repair 


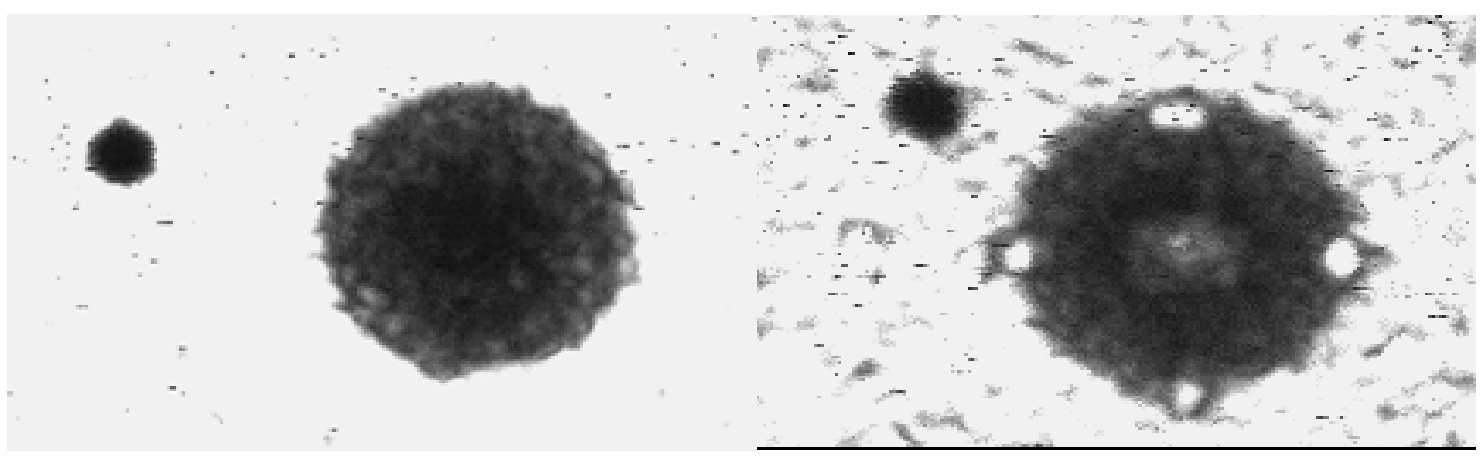

Figure 37: TTU C-scan image of S2-FM94-15 with (right) and without (left) 5-Hole Repair

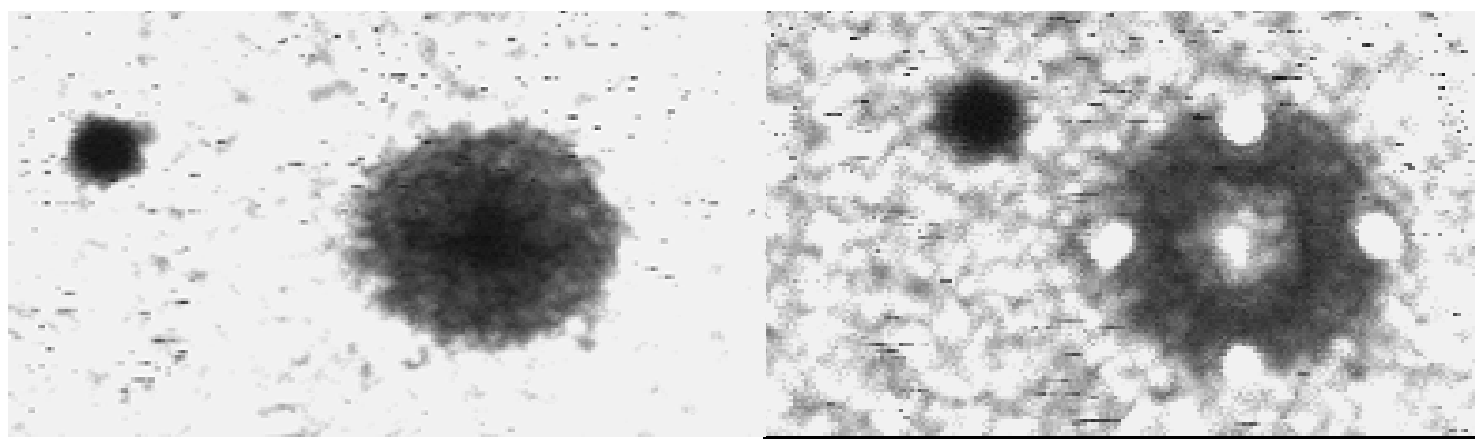

Figure 38: TTU C-scan image of E2-H-08 with (right) and without (left) 5-Hole Repair

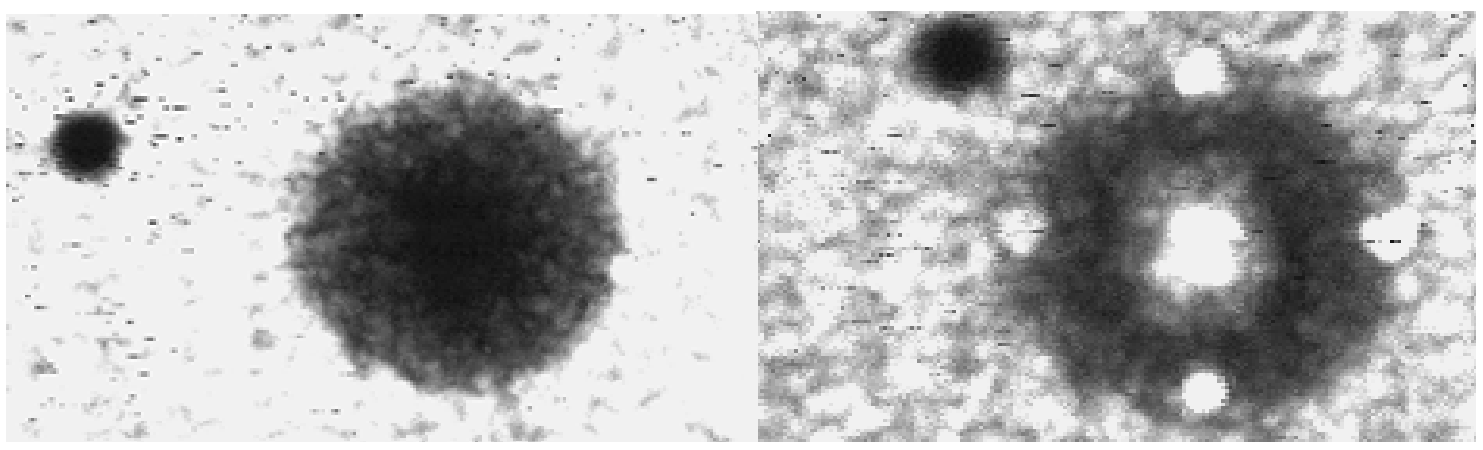

Figure 39: TTU C-scan image of E2-H-15 with (right) and without (left) 5-Hole Repair

The TTU C-scan images provide a measure of the planar distribution of the resin fills in the repaired specimens. However, they do not provide any information on the extent of the cells filled across the thickness and any resin seepage into the neighboring cells. To visualize the nature of the resin fills, a limited number of repaired sandwich specimens were sectioned 
destructively. The cross-sectional images of the repaired specimens are illustrated in Figures 40 through 44. The sectioned specimens reveal several features of the resin fill repairs. The cells filled with resin may not be completely filled across the core thickness as illustrated in Figures 42 through 44 . This could occur due to the inability to displace the air trapped in the honeycomb cell during the filling process or due to the volatiles dissipated during the curing process. In addition, the resin leakage into neighboring cells through the cell wall cracks/tears may starve the intended cell of resin. The inter cell leakage of resin was observed to occur across the entire core thickness in specimens with thinner core (Figures 40 and 41) while the leaked resin tends to settle at the bottom of the neighboring cells in sandwich specimens with thicker core (Figures 42, 43 and 44). Further, in the partially filled cells (due to air entrapment) as shown in Figure 40, the upper portion of the cell (adjacent to indented facesheets) is filled.

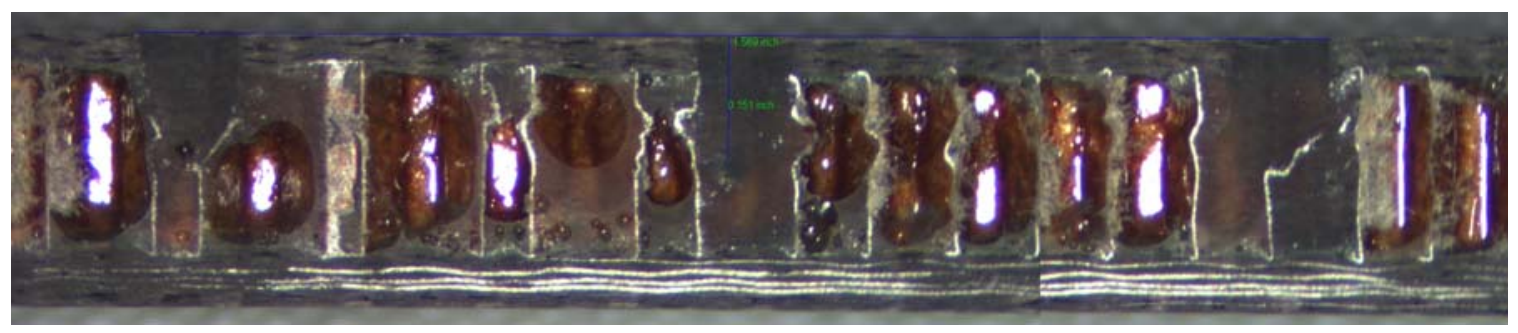

Figure 40: Destructive sectioning of E2-H-17

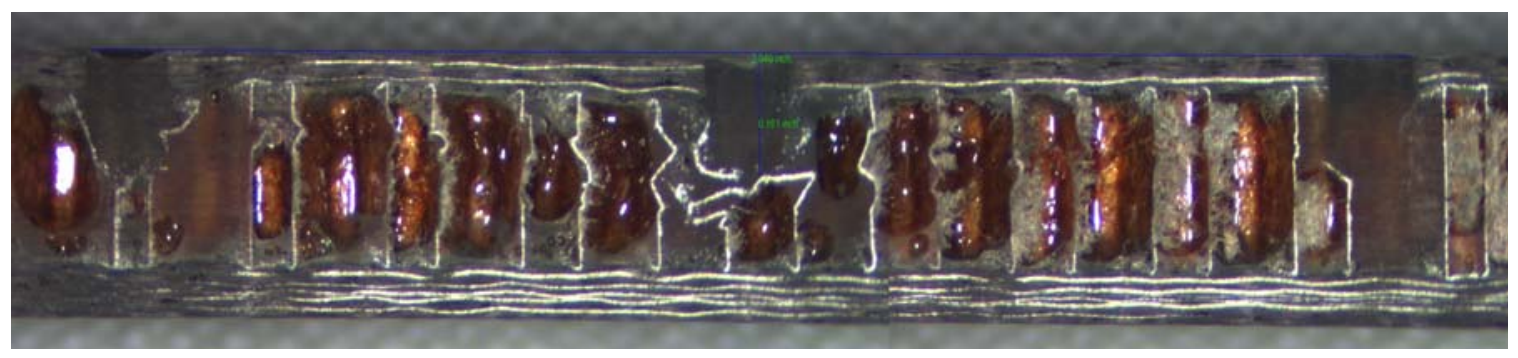

Figure 41: Destructive sectioning of E2-H-19 


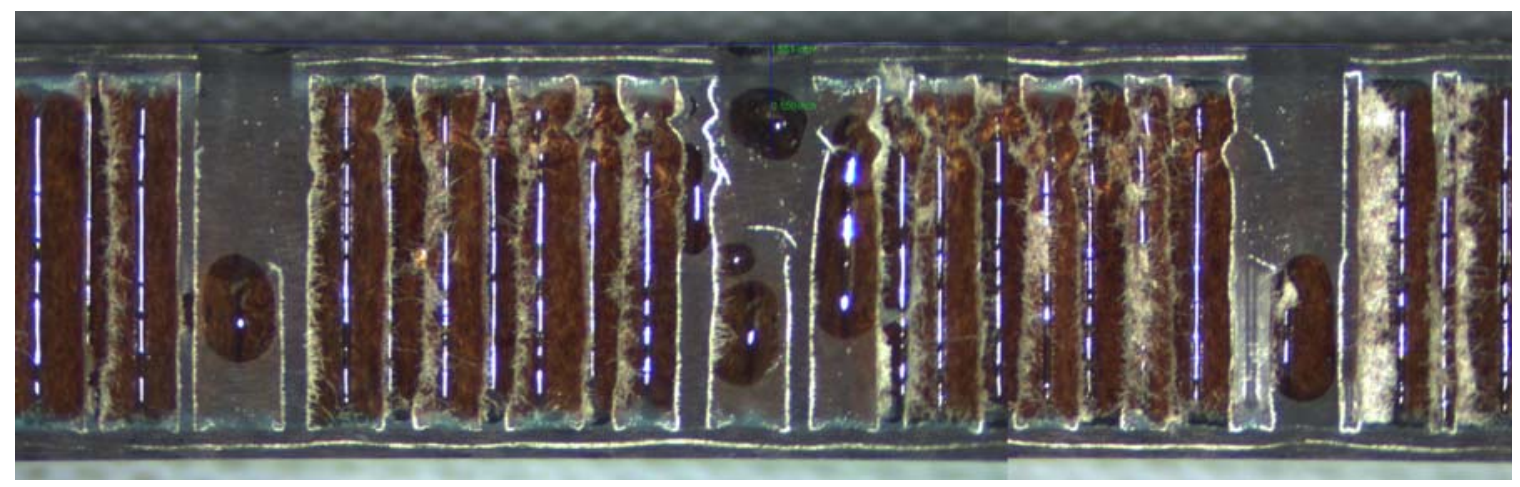

Figure 42: Destructive sectioning of S2-FM94-P17

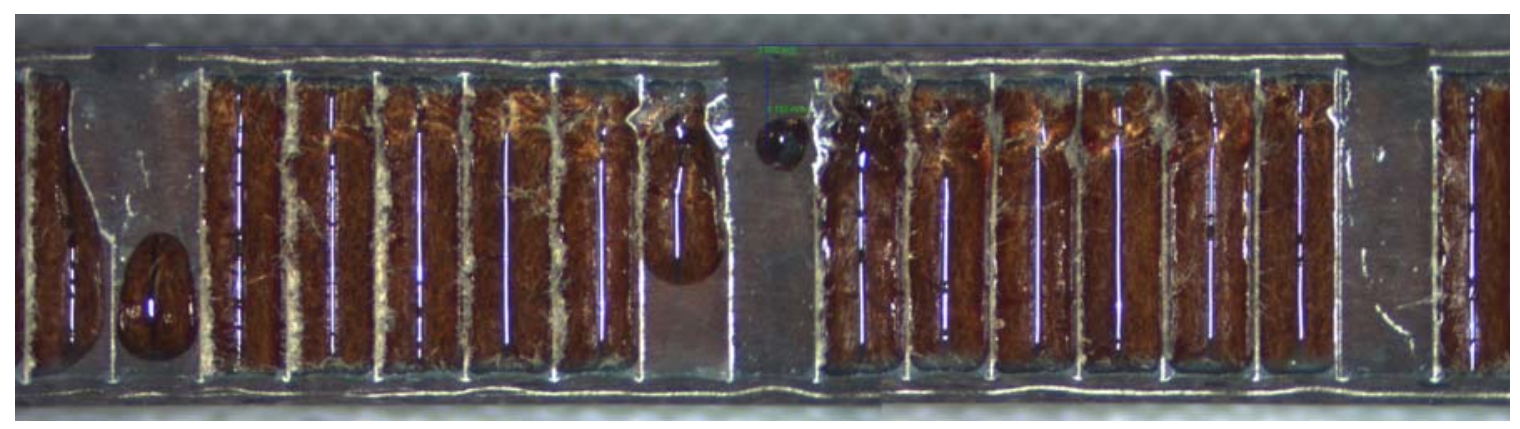

Figure 43: Destructive sectioning of S2-FM94-P19

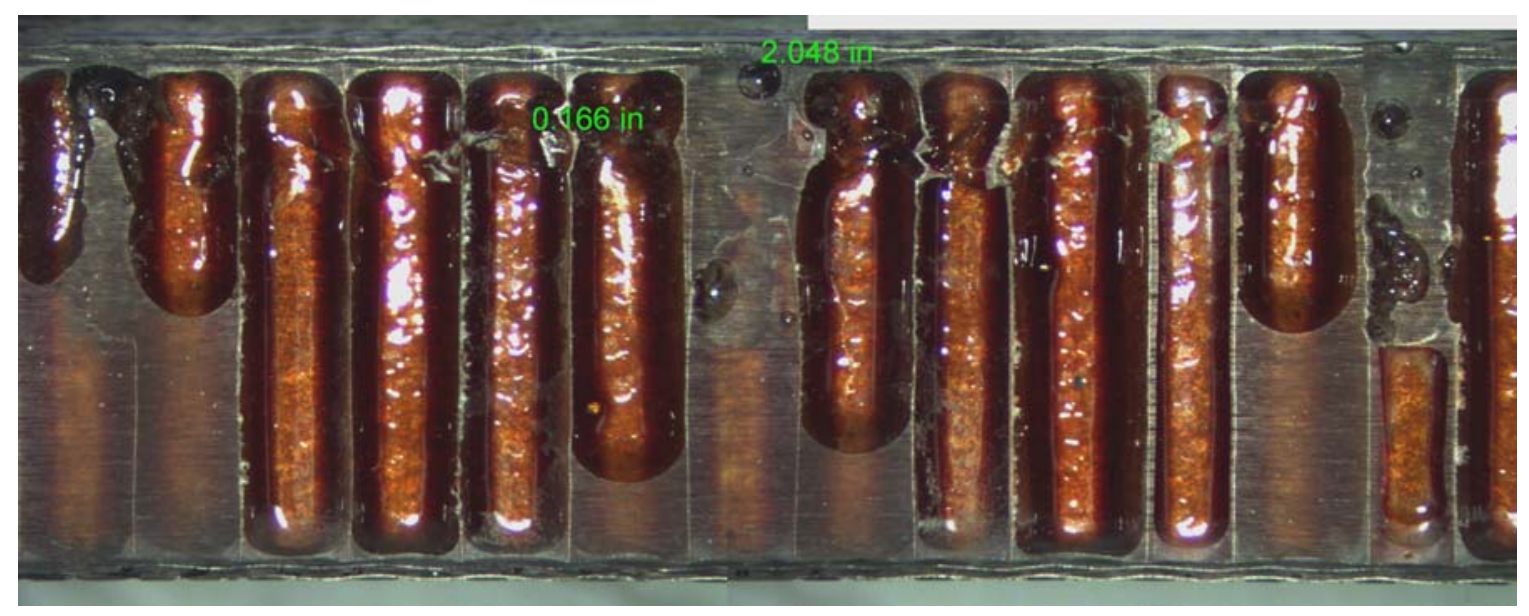

Figure 44: Destructive sectioning of CRF-11

\subsection{Characterization of Repair Effectiveness using Edgewise Compression Tests}

The effectiveness of the repair was characterized using edgewise compression tests described in the previous chapter. The behavior of the undamaged, damaged and repaired specimens was compared using the load versus end-shortening plots and the load versus out-of- 
plane displacement (at center of damage region) plots. The effectiveness of the repair was characterized in terms of the edgewise compression strength and a "Repair Effectiveness factor" [7]. In addition, the observed failure modes in the undamaged, damaged, and repaired specimens were compared.

The end shortening response of undamaged, damaged and repaired specimens was obtained by plotting running load (edge load per unit width) versus end shortening. The running load stroke response of three configurations for all the tested specimens are illustrated in Figures 45 through 50. All the three specimens in each sandwich configuration exhibit similar plots. Figure 45 illustrates the running load versus end shortening response of different configurations (undamaged, damaged and repaired) of S1-FM94 sandwich specimens. These specimens exhibit a pronounced non-linear behavior which is due to the pure off-axis nature of the facesheets which contain two $45^{\circ}$ plies. It may be observed that all specimens fail after the response has crossed over into the non-linear region. No difference could be observed during the initial stages of loading, i.e., the elastic responses were unaffected by the presence of damage/repair. The maximum load of damaged specimen is found to be decreased from its undamaged condition due the presence of impact damage on one of its facesheet. The three repair configurations used for this sandwich type exhibited higher loads at failure indicating an improvement in compressive properties. However, the repair configuration with two filled cells at the edge of the damage region proved to be marginally better than the damaged specimen as shown in Figure 45. The repair configurations (1-hole and 3-hole repair) where a core cell in the center of the damage was filled, increased the strength of the panel close to (or exceeding) the undamaged panel strength. Thus, it was realized that the reinforcement of the cell in the center of the damage region is 
required to obtain substantial benefits from the repair. Thus, the five-hole repair method was chosen for the other sandwich configurations (S2-FM94, CRF and E2-H).

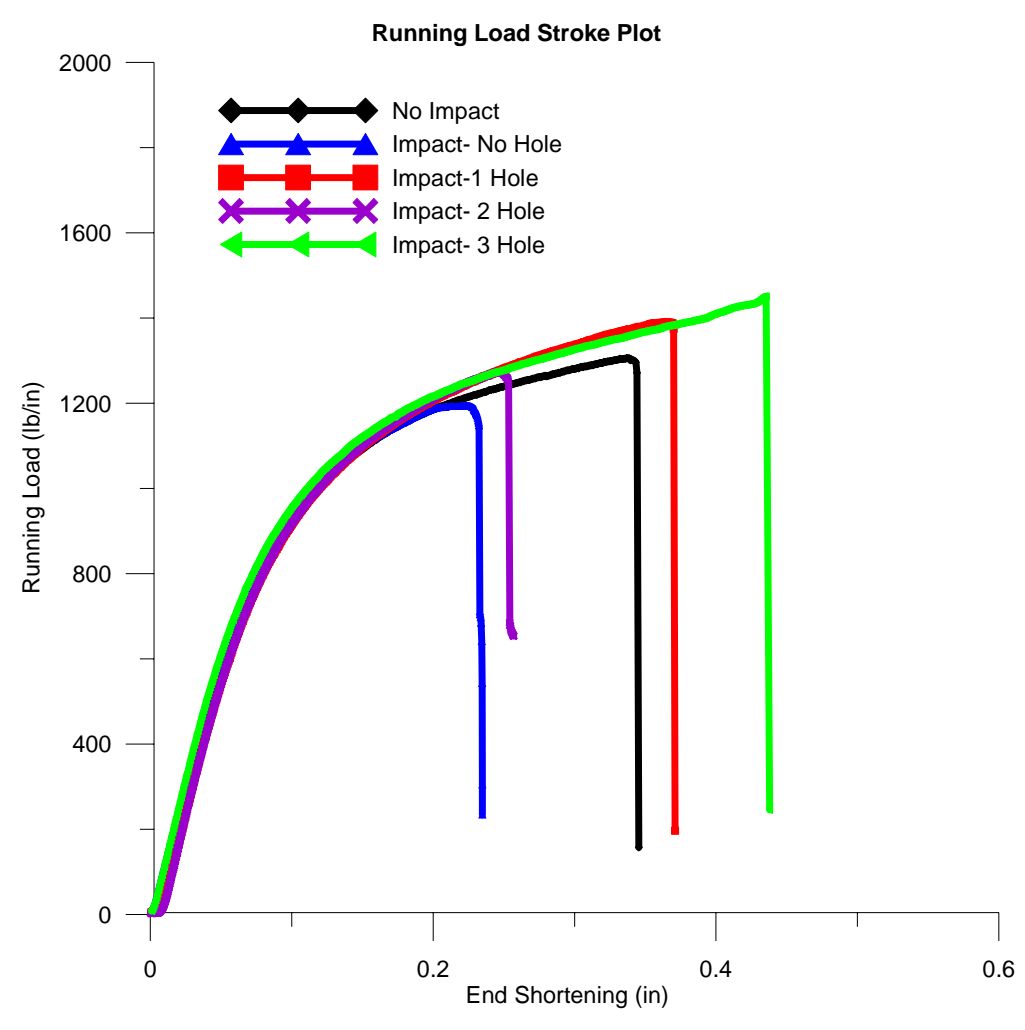

Figure 45: Running Load versus End Shortening of S1-FM94

The end shortening response of undamaged, damaged and repaired specimens was obtained by plotting running load (lb/in) versus end shortening (in) as illustrated in Figures 45 through 50. The running load stroke response of three configurations for all the tested specimens are illustrated in Figures 45 through 50. S2-FMX0.10 and E2-HX0.10 specimens exhibit similar nature of end shortening responses for all the three configurations as shown in Figures 46 and 47. These sandwich configurations exhibit a linear response due to the presence of $0^{\circ}$ plies in the facesheets. The figures indicate that no significant differences in stiffness exist between the damaged, undamaged and repaired sandwich specimens. However, the compressive strength of the repaired panels was consistently higher than that of the damaged panels indicating an effective repair. 


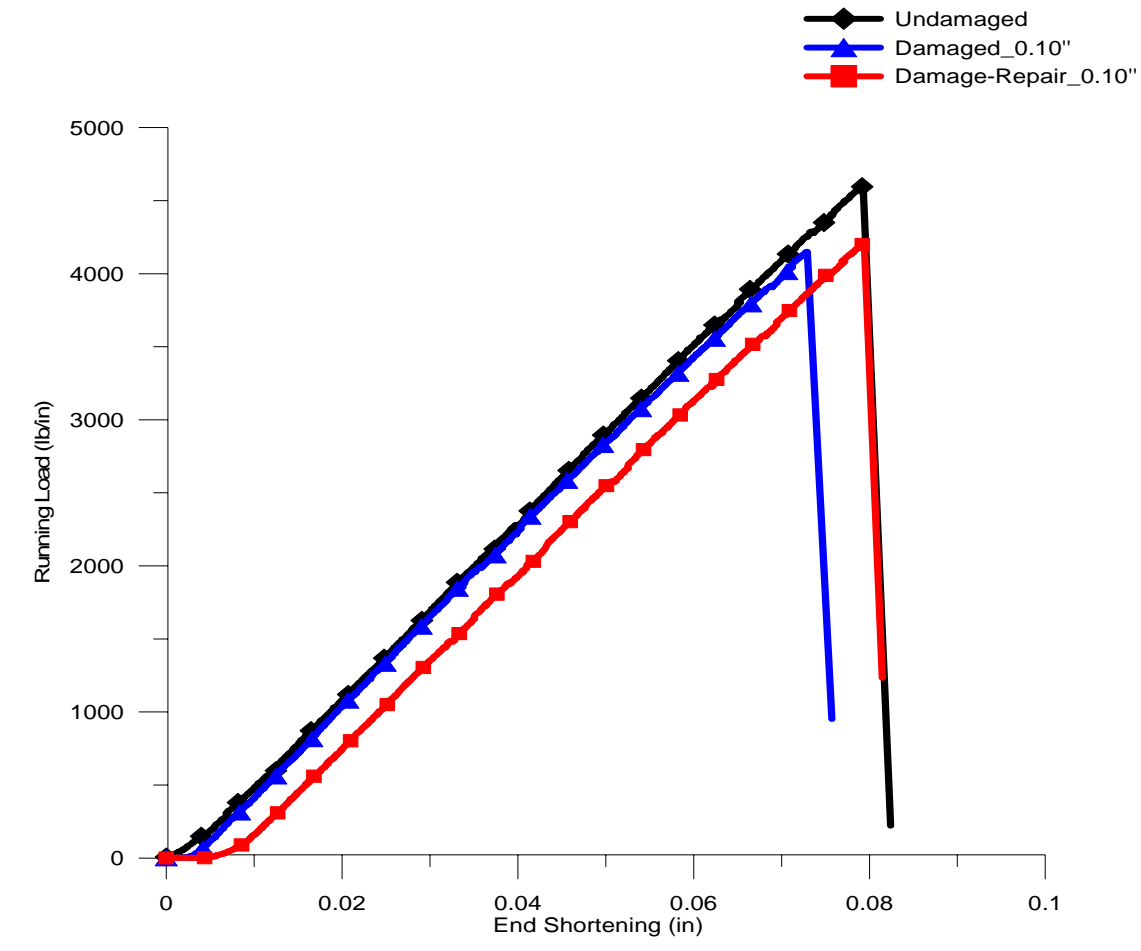

Figure 46: Running load versus end shortening of E2-HX0.10 specimens

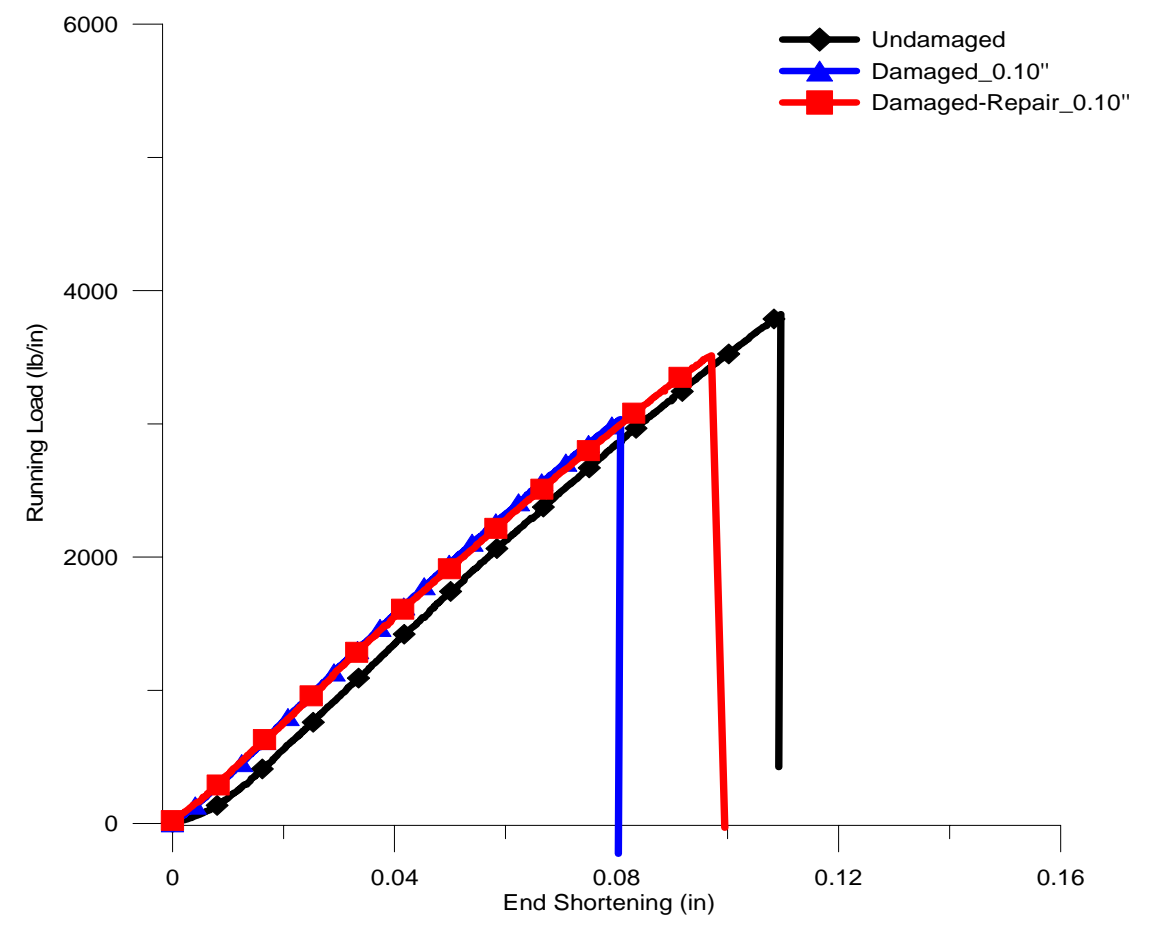

Figure 47: Running load versus end shortening of S2-FM94X0.10 specimens 


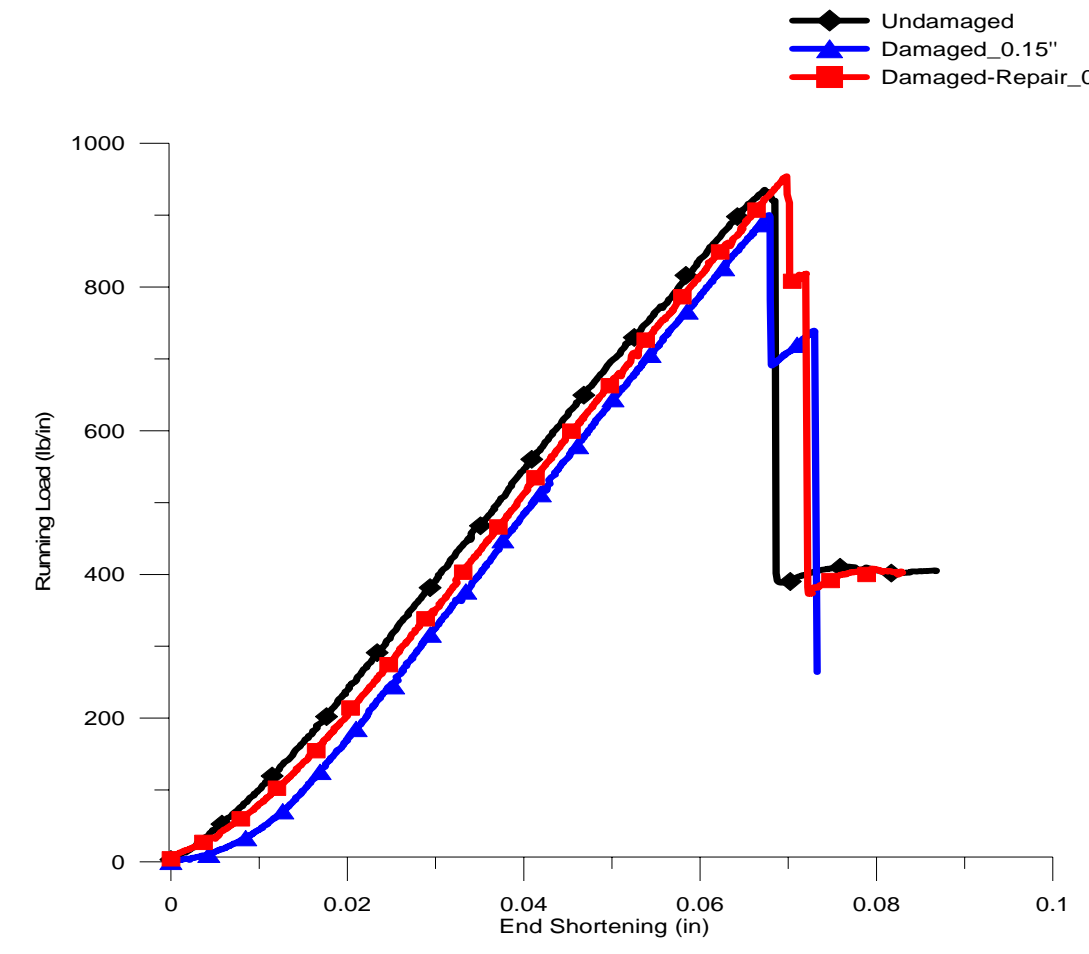

Figure 48: Running load versus end shortening of CRFX0.15 specimens

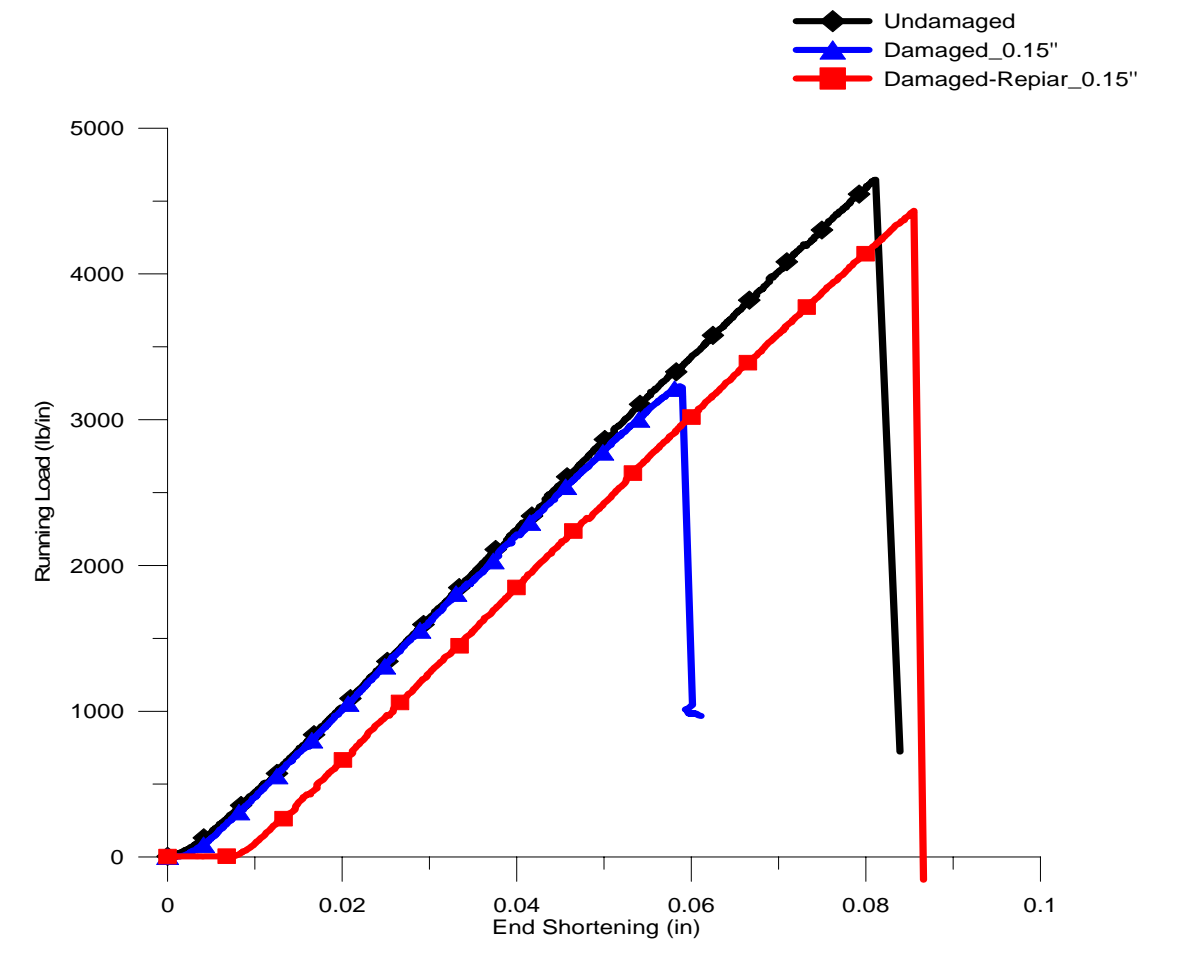

Figure 49: Running load versus end shortening of E2-HX0.15 specimens 


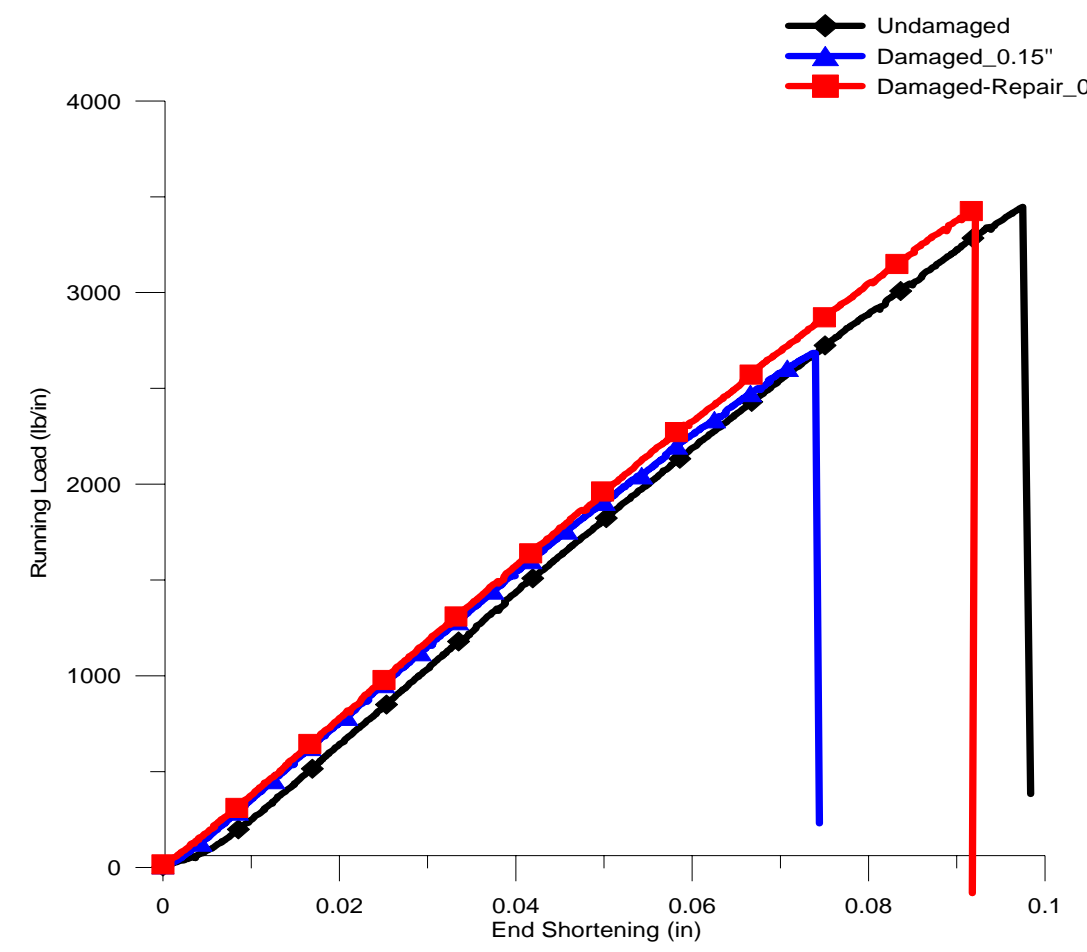

Figure 50: Running load versus end shortening of S2-FM94X0.15 specimens

\subsubsection{Measurement of Local Bending}

The load-end shortening behavior of undamaged, damaged and repaired sandwich panels provides an overall picture of the effectiveness of repair. However, they do not provide any information about the repair effectiveness in containing the local bending of the facesheet which was its primary purpose. The localized bending of the facesheet was measured using an LVDT as described in the previous chapter. The LVDT measures the out-of-plane displacement at the center of the damage zone and indicates the amount of local facesheet bending over the damaged core.

The running load versus out-of-plane displacement responses of damaged and repaired configurations of S1-FM94 sandwich specimens are plotted in Figure 51. The damaged specimen indicates a significant amount of local bending of the impacted/indented facesheet over the damaged core. The sandwich specimen repaired by filling of two cells at the edge of the damage 
region delayed the onset of local bending but could not contain it completely as shown in the same figure. However, the repair configurations where the core cell at the center of the damage region was filled exhibit containment of the local bending. Based on this observation, it is necessary that the core cell at the center of the damage region must be filled in order to successfully contain local bending and improve the strength of the damaged panels.

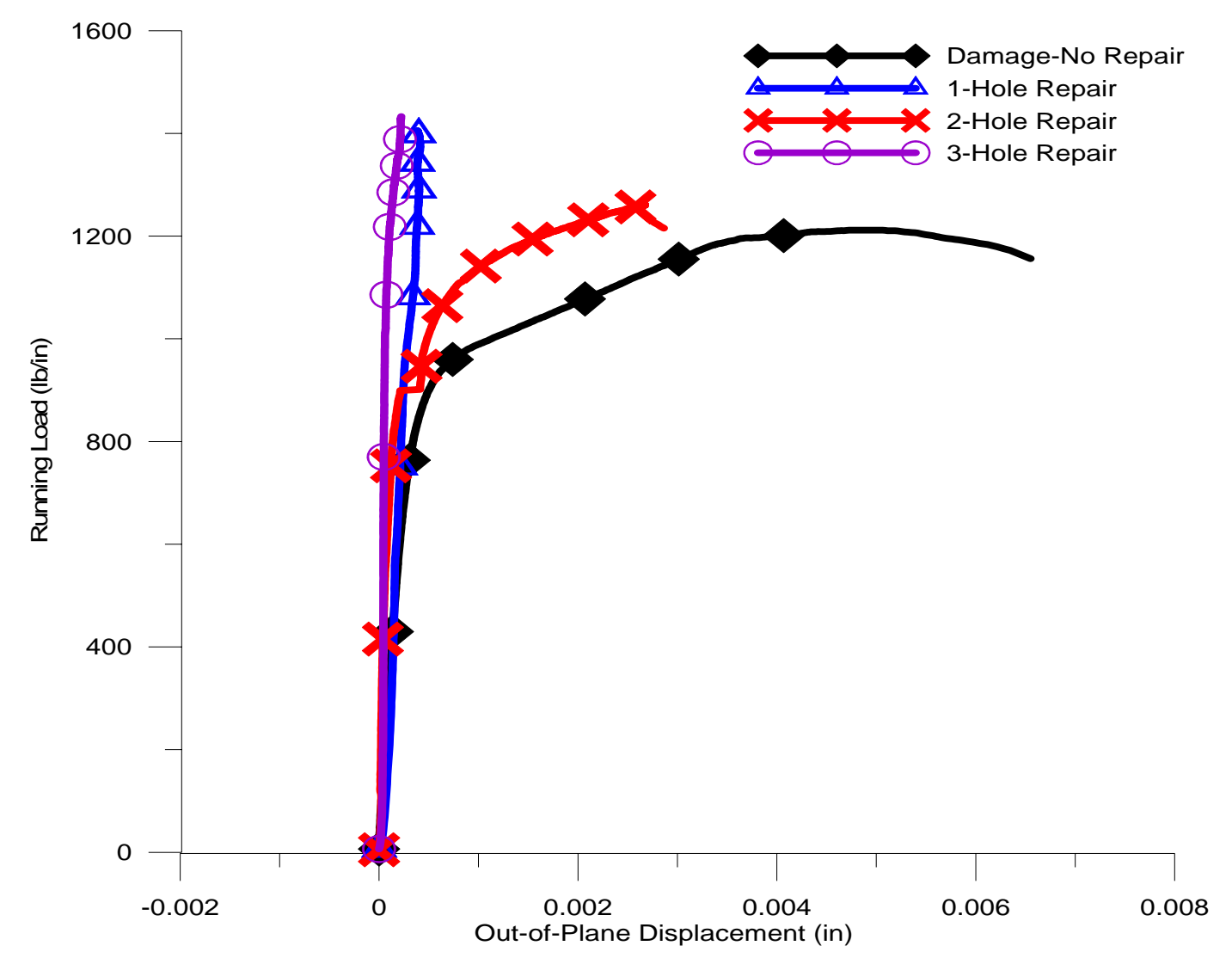

Figure 51: Running load versus out-of-plane displacement of S1-FM94 Sandwich

Similarly, the localized bending effects of three configurations such as undamaged, damaged and repaired specimens of S2-FM94, CRF and E2-H sandwich types are illustrated in Figures 52 through 56. It is observed that global bending exists in undamaged E2-H specimens due to the unsymmetrical sandwich construction. This sandwich type is composed of 10- 
ply/core/6-ply sequence. Due to the five-hole repaired configuration, the local buckling effects of E2-H specimens were improved and are found to be contained. In case of S2-FM94 and CRF specimens, the global bending and/or buckling of the specimens is not present due to their symmetrical construction. The undamaged specimens exhibit negligible local bending whereas the damaged specimens were under the influence of local bending as illustrated in Figures 53, 54 and 56. The magnitude of local bending was proportional to the amount of damage inflicted on the specimens. However, the repaired specimens exhibited reduced levels of facesheet bending as evident from Figures 53, 54 and 56. Thus, the repair method is instrumental in containing the local bending of damaged facesheets and thus increasing the in-plane compressive strength of damaged panels.

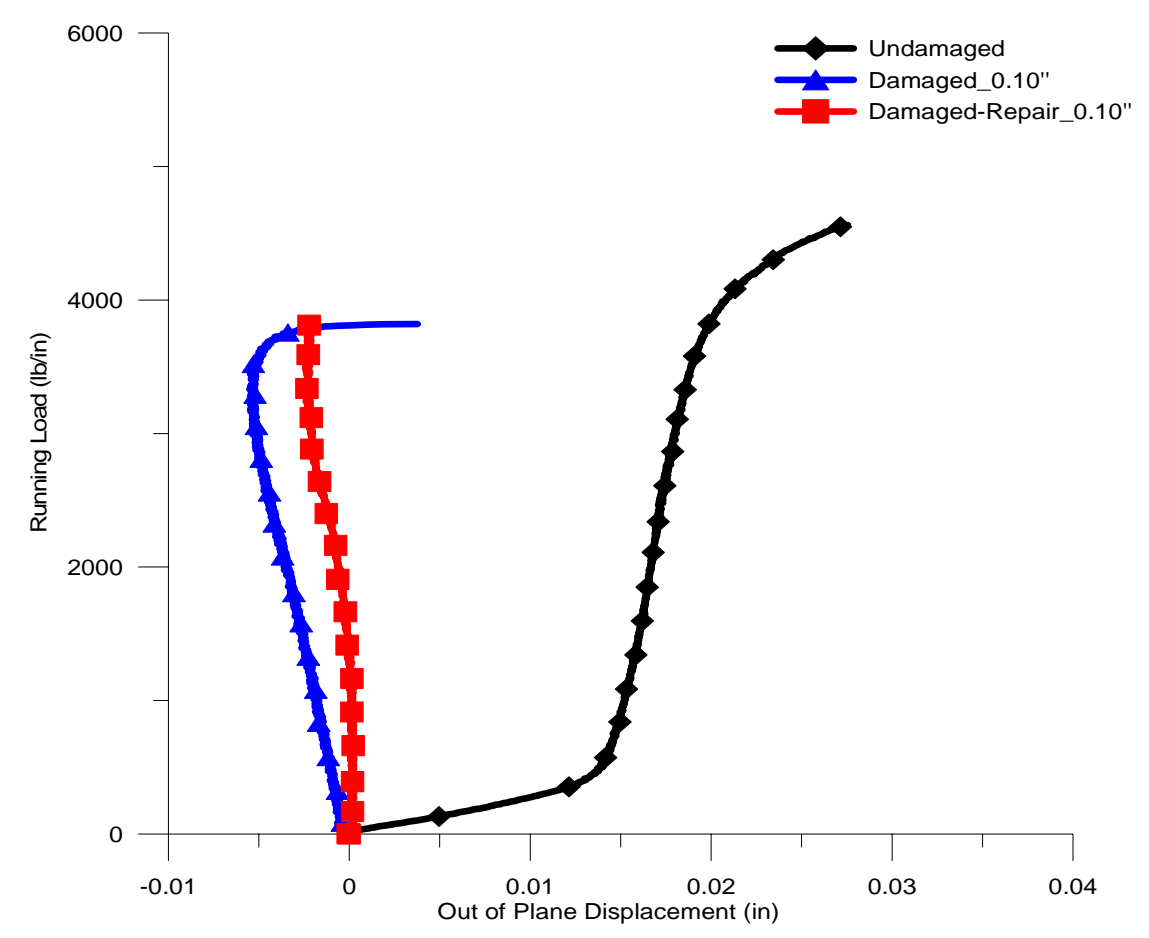

Figure 52: Running load versus out-of-plane displacement of E2-HX0.10 specimens 


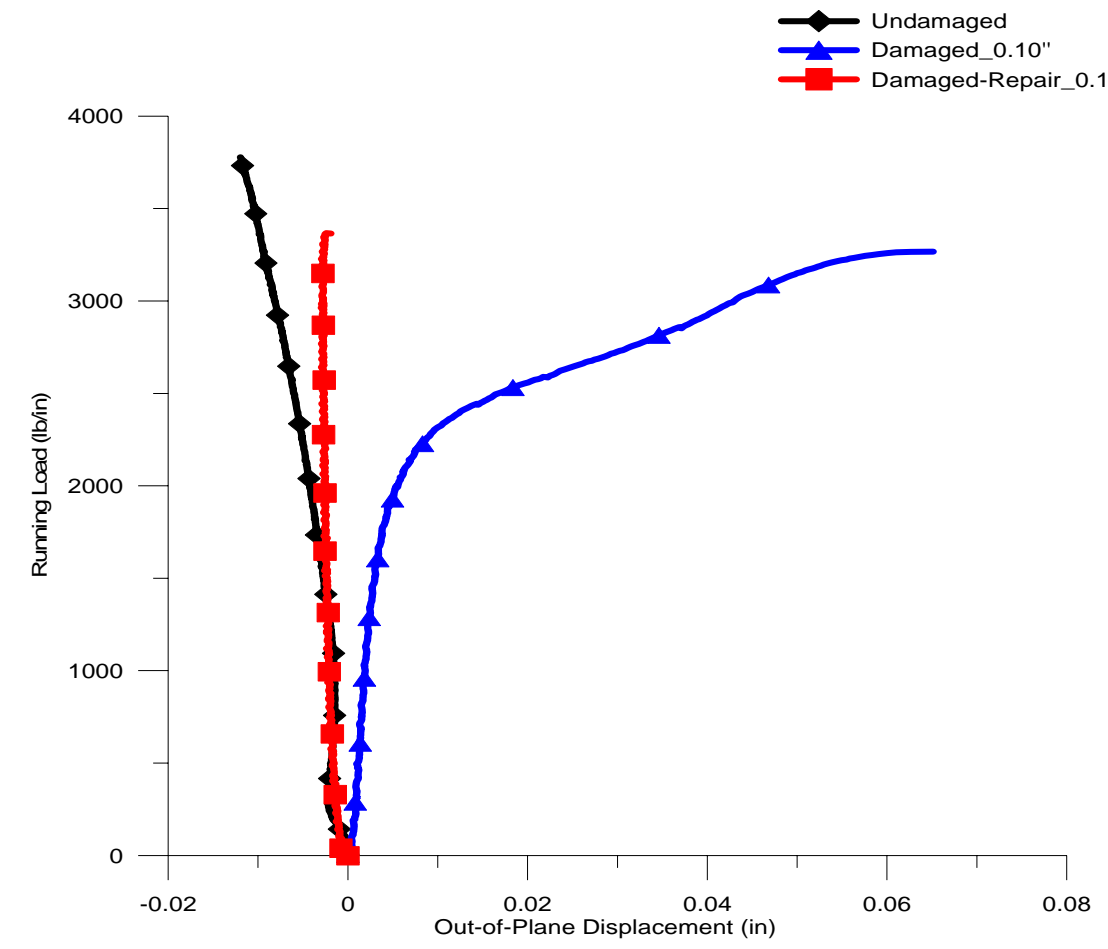

Figure 53: Running load verses out-of-plane displacement of S2-FM94X0.10 specimens

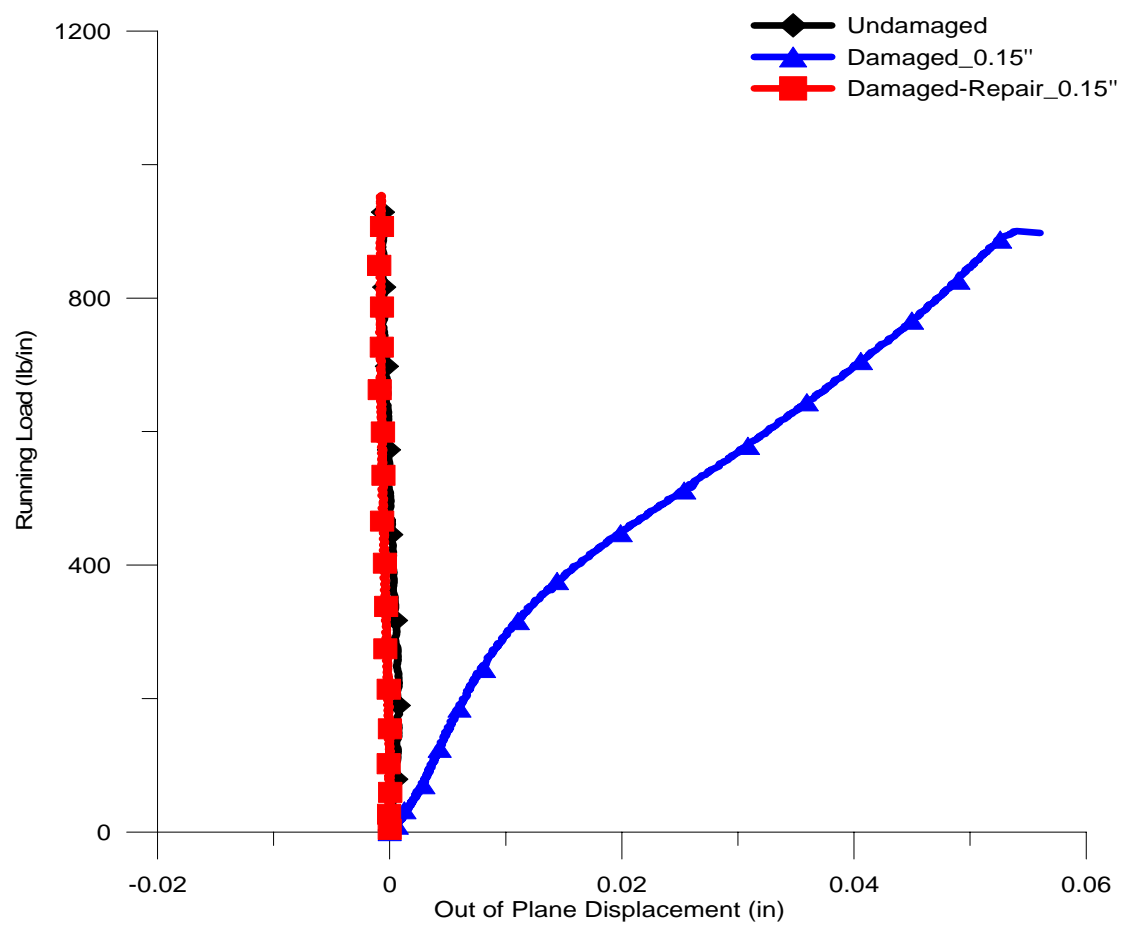

Figure 54: Running load versus out-of-plane displacement of CRFX0.15 specimens 


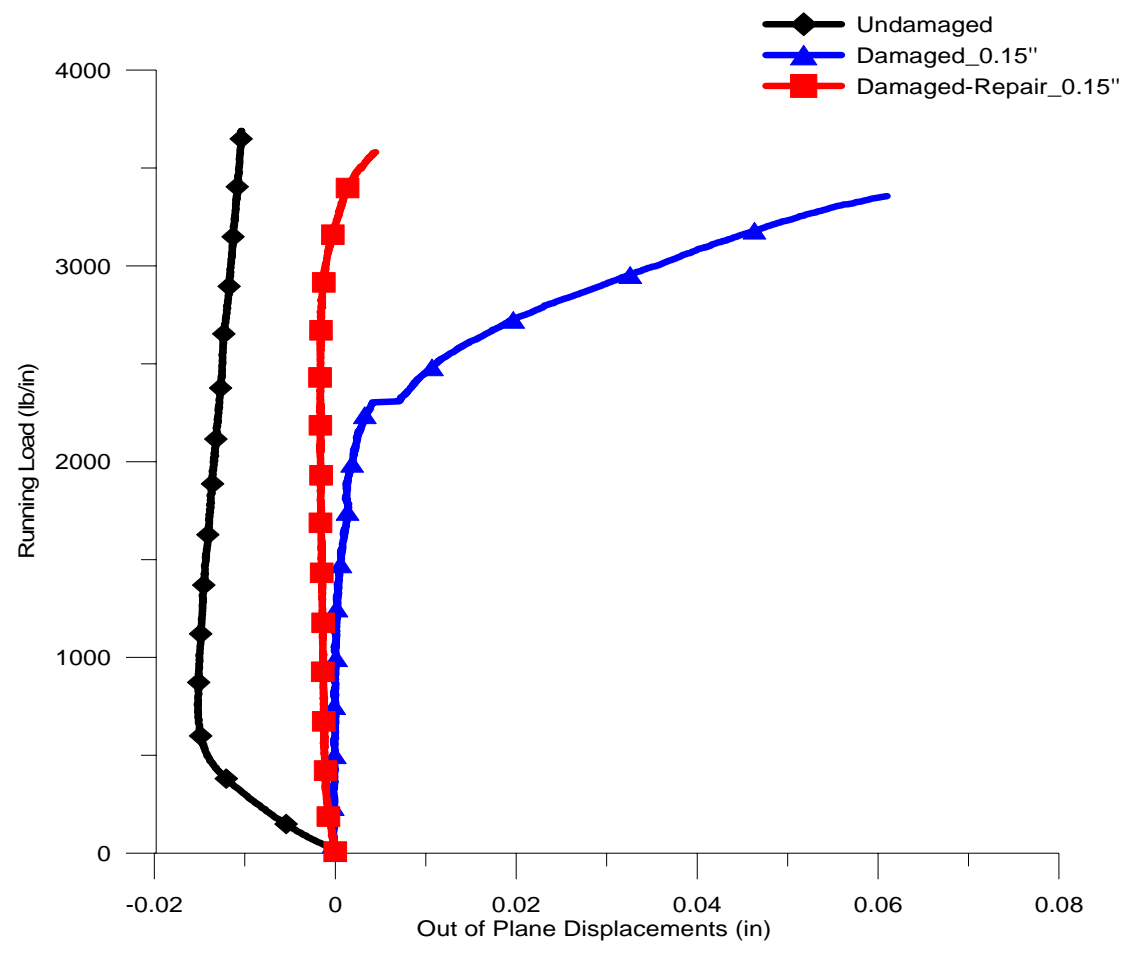

Figure 55: Running load versus out-of-plane displacement of E2-HX0.15 specimens

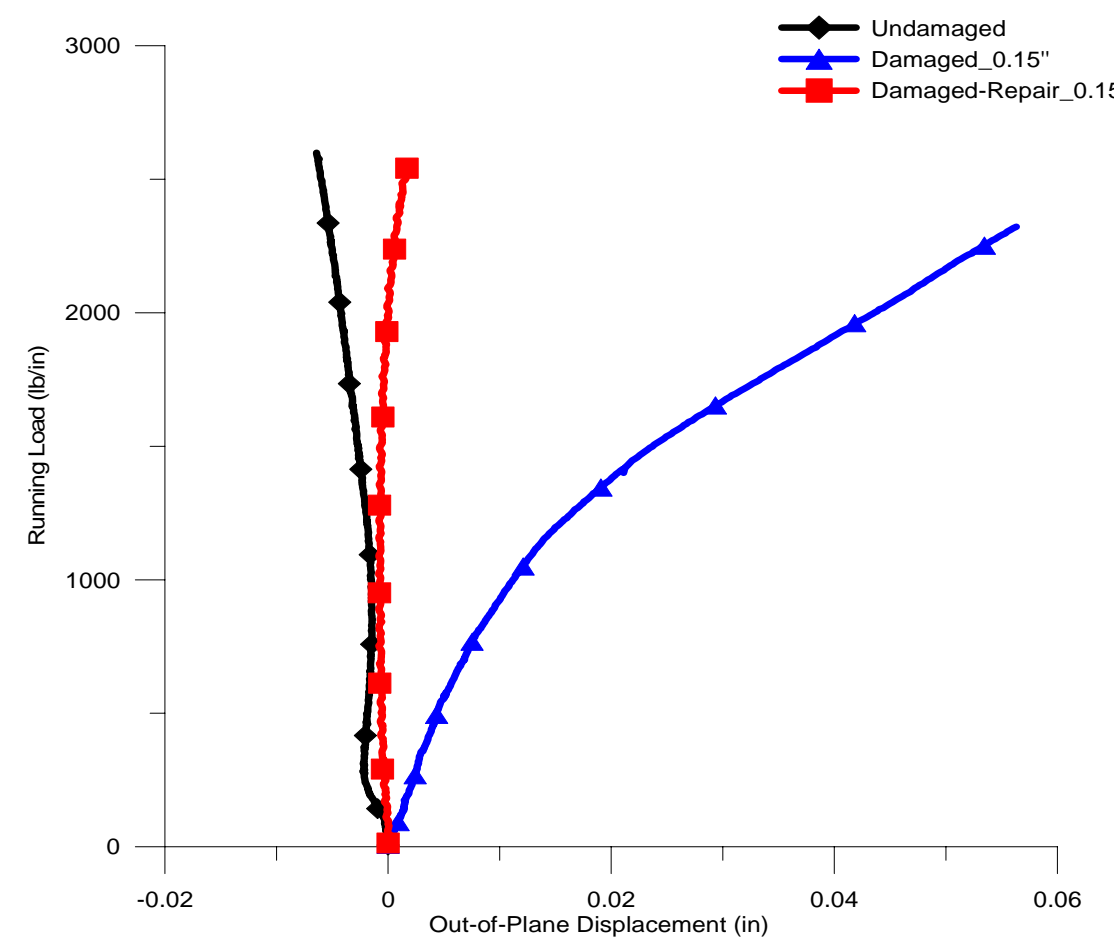

Figure 56: Running load versus out-of-plane displacement of S2-FM94X0.15 specimens 


\subsubsection{Edgewise Compression Strengths}

The end-shortening and local bending responses provided a qualitative assessment of the effects of damage and repair on the in-plane compressive strength of sandwich specimens. The effectiveness of the repair process was quantified in terms of the in-plane compressive strength of the sandwich specimens. The in-plane compressive strength corresponds to the maximum load sustained by the specimen prior to failure.

The in-plane compressive strength comparison for S1-FM94 specimens with different repair and/or damage schemes are summarized in Figure 57. The compressive strength of damaged specimen is reduced to a noticeable amount due to the presence of impact damage whereas the strength of one-hole repair was found to be more than the undamaged configuration as the center of the damage region was reinforced with resin repair. The strength of two-hole repair configuration was found to be less than that of one-hole repair as the two holes on the circumference placed laterally are repaired with resin mixture. Thus, the reinforcement at the center of the damage area is not present in two-hole repair method. Three-hole repair configuration is an extension of two-hole repair method, with additional repair hole at the center of the damage region to provide strength enhancement and lateral direction in the plane of specimen. The three-hole repair configuration was observed to restore/exceed the strength to the undamaged value. The average value of compression-after-impact strength (lb/in) of three configurations: undamaged, damaged and damaged-repair of S2-FM94, CRF and E2-H material systems with subsurface core damage are compared in Figures 58 through 62. It is noticed that the strength of damaged specimens decreases when compared to undamaged specimens. The magnitude of compressive strength reduction was proportional to the amount of damage inflicted on the specimens. However, the five-hole repair configuration was observed to restore the 
strength close to the undamaged value. A noticeable increase in compressive strength can be observed for resin repaired specimens.

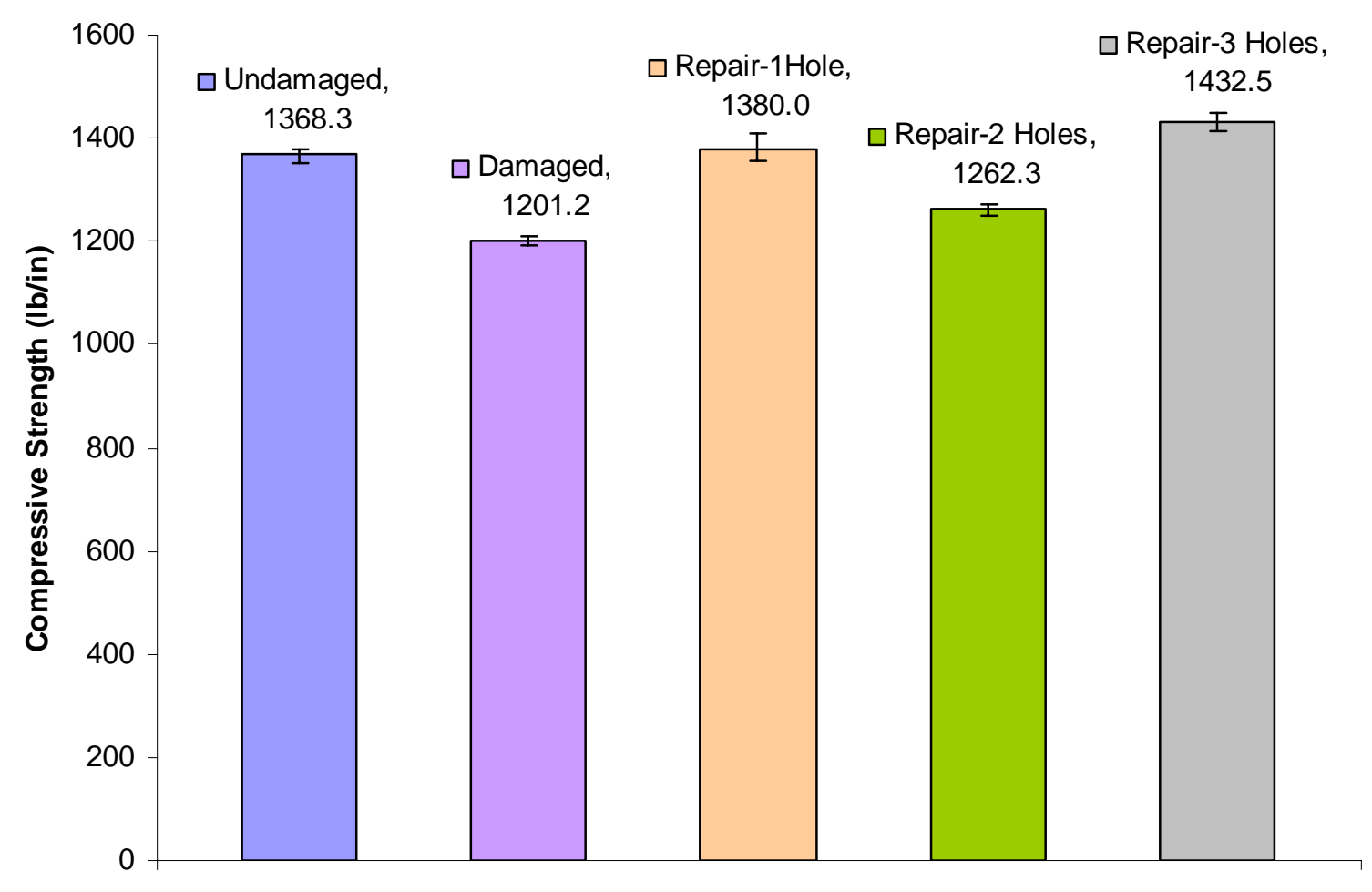

Figure 57: Comparison of Strengths of S1-FM94 sandwich material system 


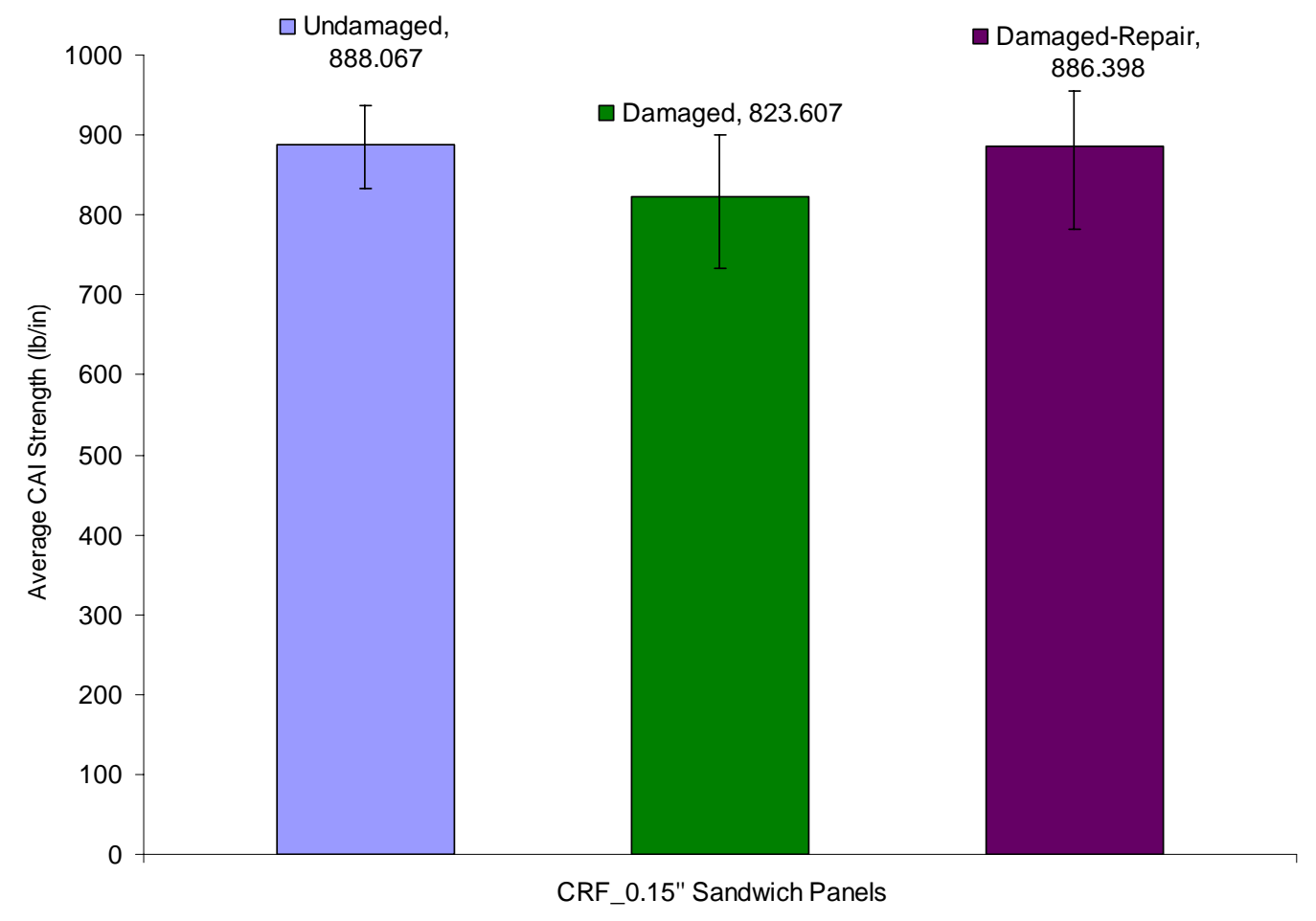

Figure 58: Strength comparisons of CRFX0.15 specimens

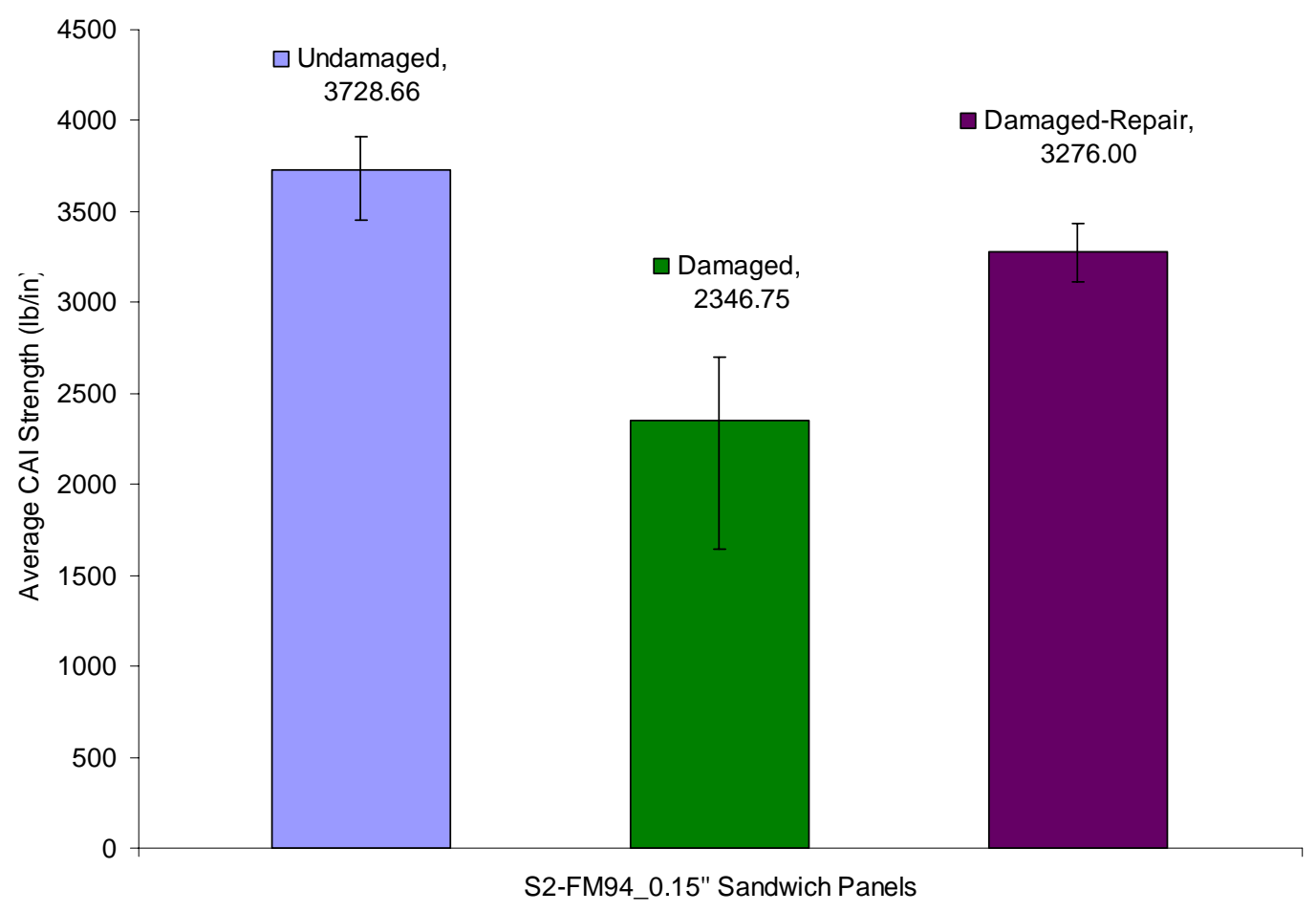

Figure 59: Strength comparisons of S2-FM94X0.15 specimens 


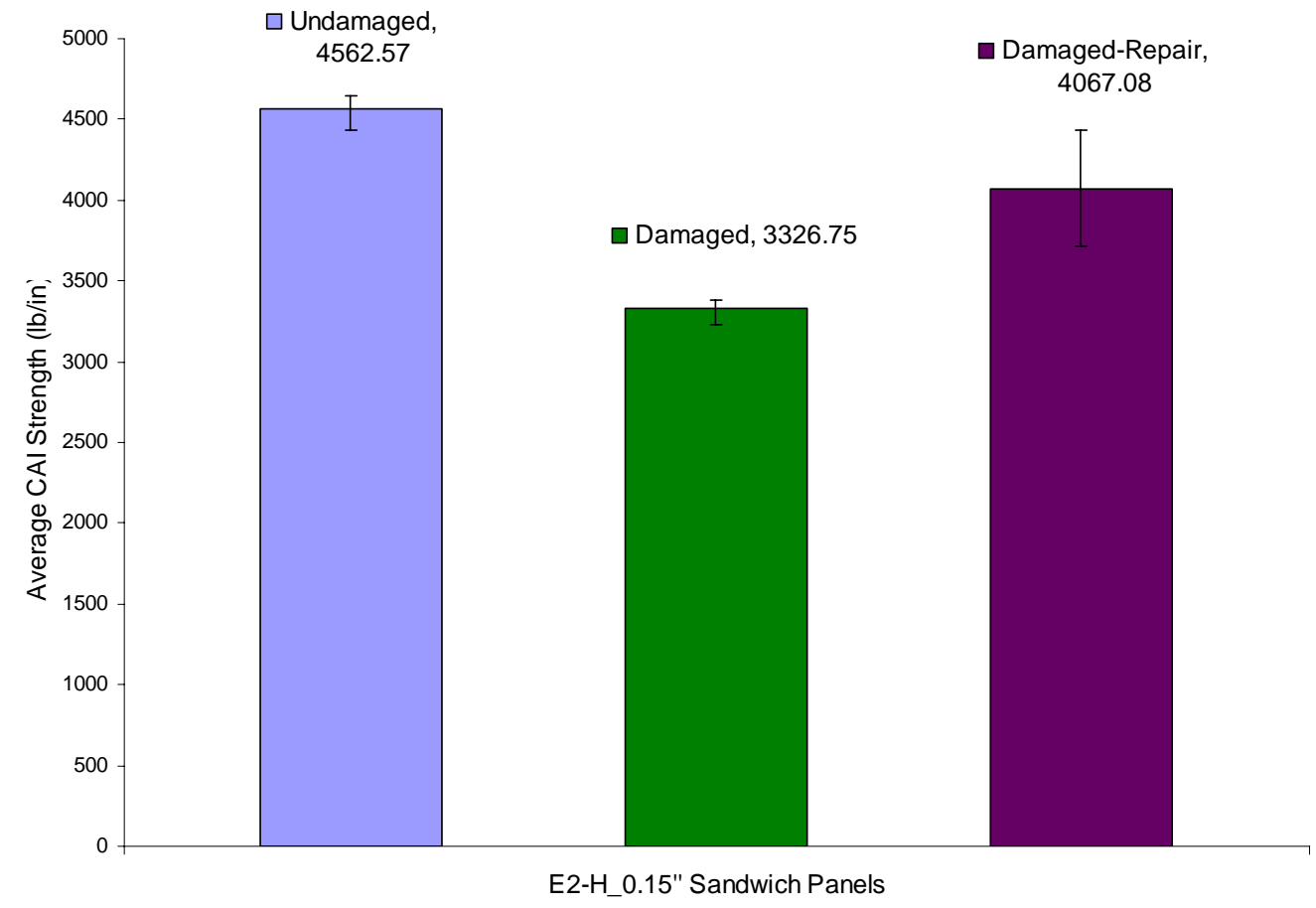

Figure 60: Strength comparisons of E2-HX0.15 specimens

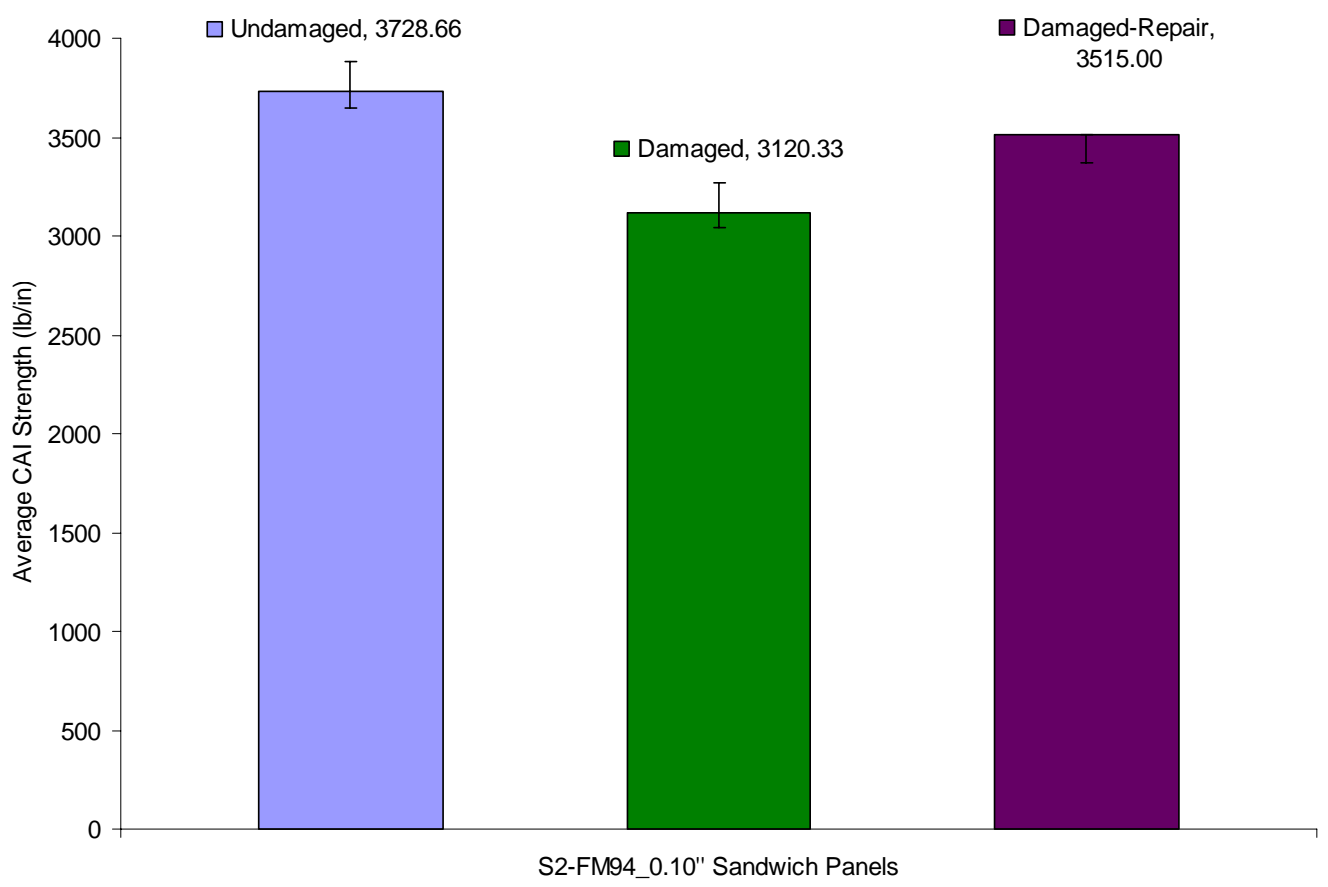

Figure 61: Strength comparisons of S2-FM94X0.10 specimens 


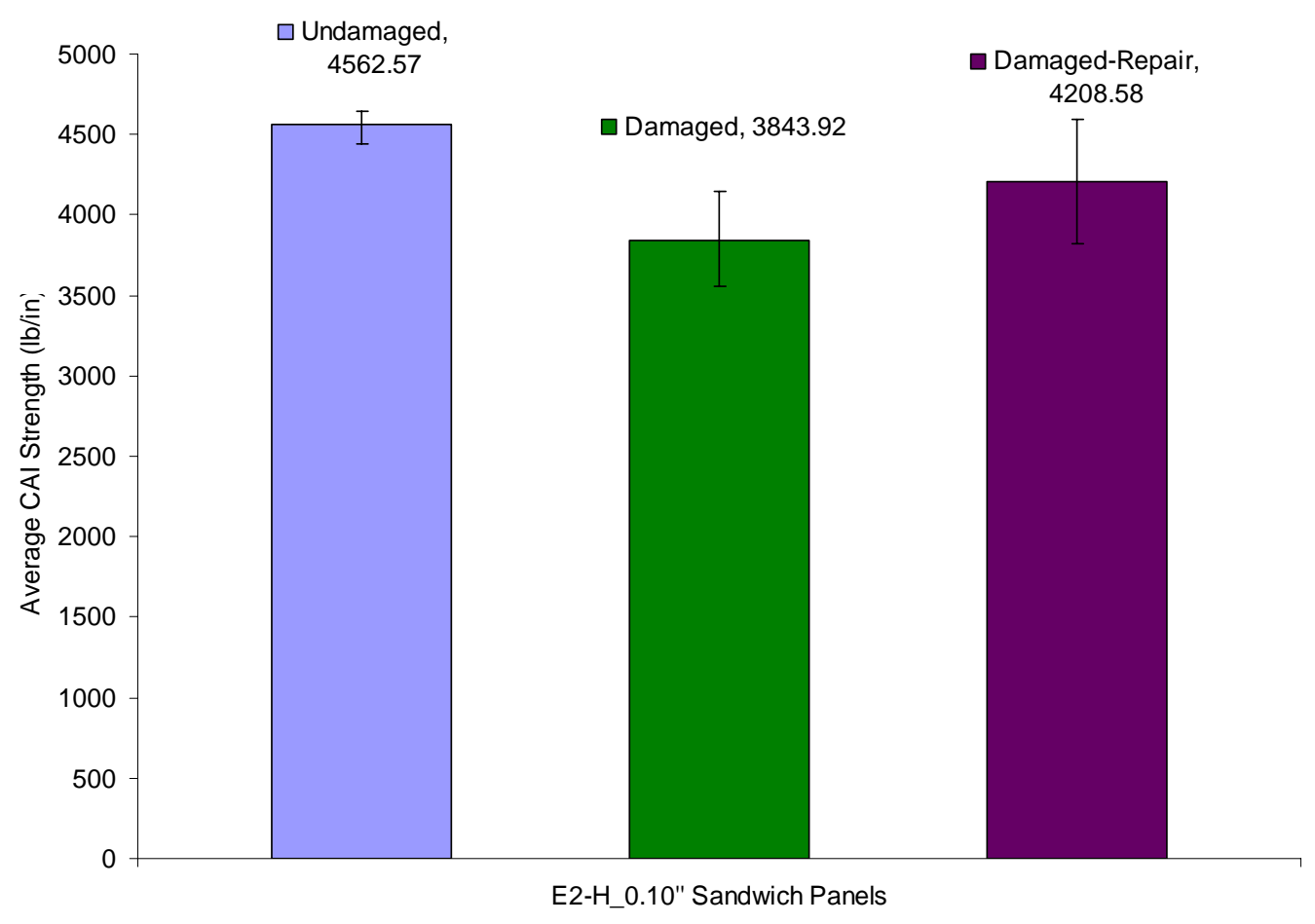

Figure 62: Strength comparisons of E2-HX0.10 specimens

Table 10 and Table 11 detail the summary of experimental results of CRF, S2-FM94, E2$\mathrm{H}$ and S1-FM94 specimens in which the maximum residual dent depth, planar damage area and CAI strength are tabulated. To support the validity of results, the average value of three specimens and coefficient of variation were added. It is evident that the data are not scattered so much validating the results. The test data indicates that the residual strength of the damaged sandwich specimens was increased to 87-99 \% of the undamaged strength using the five-hole repair configuration. The repair effectiveness and the strength improvement due to repair are tabulated in Table 12. The repair effectiveness is defined as the ratio of difference between repair and damaged strengths to the difference between virgin and damaged strengths. The repair was observed to be more effective (0.97) in CRF specimens when compared to S2-FM94 and E2-H 
specimens ( 0.51 to 0.67$)$. Thus, the CRF specimens are most effective in improving the residual strength of the damaged configuration.

TABLE 10

SUMMARY OF RESULTS: COMPRESSIVE STRENGTH

\begin{tabular}{|c|c|c|c|c|c|}
\hline Panel Type & Configurations & $\begin{array}{l}\text { Indenter/ } \\
\text { Impactor } \\
\text { Diameter }\end{array}$ & $\begin{array}{c}\text { Indentation } \\
\text { Depth/Impact } \\
\text { Energy }\end{array}$ & $\begin{array}{c}\text { CAI } \\
\text { Strength } \\
\text { (lb/in) }\end{array}$ & Avg.* $\left(\mathrm{CV}^{* *}\right)$ \\
\hline CRF-1 & \multirow{3}{*}{ UD } & $3.00 "$ & \multirow{3}{*}{ N/A } & 936.21 & \multirow{3}{*}{ 888.07(5.85) } \\
\hline CRF-2 & & $3.00 "$ & & 895.05 & \\
\hline CRF-3 & & $3.00 "$ & & 832.94 & \\
\hline CRF-4 & \multirow{3}{*}{ DNR } & $3.00 "$ & \multirow{3}{*}{$0.15 "$} & 733.43 & \multirow{3}{*}{ 823.61(10.26) } \\
\hline CRF-5 & & $3.00 "$ & & 836.40 & \\
\hline CRF-6 & & $3.00^{\prime \prime}$ & & 901.00 & \\
\hline CRF-7 & \multirow{3}{*}{ DR } & $3.00 "$ & \multirow{3}{*}{$0.15 "$} & 955.71 & \multirow{3}{*}{$886.40(10.44)$} \\
\hline CRF-8 & & $3.00 "$ & & 781.38 & \\
\hline CRF-9 & & $3.00 "$ & & 922.11 & \\
\hline S2-FM94-20 & \multirow{3}{*}{ UD } & $3.00 "$ & \multirow{3}{*}{ N/A } & 3824.34 & \multirow{3}{*}{ 3728.66(6.57) } \\
\hline S2-FM94-2 & & $3.00 "$ & & 3450.50 & \\
\hline S2-FM94-3 & & $3.00 "$ & & 3911.15 & \\
\hline S2-FM94-4 & \multirow{3}{*}{ DNR } & $3.00 "$ & \multirow{3}{*}{$0.10^{\prime \prime}$} & 3038.75 & \multirow{3}{*}{$3120.33(4.17)$} \\
\hline S2-FM94-5 & & $3.00 "$ & & 3051.75 & \\
\hline S2-FM94-6 & & $3.00 "$ & & 3270.50 & \\
\hline S2-FM94-7 & \multirow{3}{*}{ DR } & $3.00 "$ & \multirow{3}{*}{$0.10^{\prime \prime}$} & 3516.75 & \multirow{3}{*}{$3467.67(2.41)$} \\
\hline S2-FM94-8 & & $3.00^{\prime \prime}$ & & 3515.00 & \\
\hline S2-FM94-9 & & $3.00 "$ & & 3371.25 & \\
\hline S2-FM94-10 & \multirow{3}{*}{ DNR } & $3.00^{\prime \prime}$ & \multirow{3}{*}{$0.15 "$} & 1648.00 & \multirow{3}{*}{$2387.75(25.79)$} \\
\hline S2-FM94-11 & & $3.00 "$ & & 2687.75 & \\
\hline S2-FM94-12 & & $3.00 "$ & & 2704.50 & \\
\hline S2-FM94-13 & \multirow{3}{*}{ DR } & $3.00 "$ & \multirow{3}{*}{$0.15 "$} & 3282.00 & \multirow{3}{*}{$3276.00(5.02)$} \\
\hline S2-FM94-14 & & $3.00^{\prime \prime}$ & & 3437.25 & \\
\hline S2-FM94-15 & & $3.00 "$ & & 3108.75 & \\
\hline E2-H-1 & \multirow{3}{*}{ UD } & $3.00 "$ & \multirow{3}{*}{ N/A } & 4601.68 & \multirow{3}{*}{$4562.57(2.42)$} \\
\hline E2-H-2 & & $3.00 "$ & & 4438.11 & \\
\hline E2-H-3 & & $3.00 "$ & & 4647.93 & \\
\hline E2-H-4 & \multirow{3}{*}{ DNR } & $3.00 "$ & \multirow{3}{*}{$0.10^{\prime \prime}$} & 4151.25 & \\
\hline E2-H-5 & & $3.00 "$ & & 3559.25 & $3843.92(7.72)$ \\
\hline E2-H-6 & & $3.00 "$ & & 3821.25 & \\
\hline E2-H-7 & & $3.00 "$ & & 4203.50 & \\
\hline E2-H-8 & DR & $3.00^{\prime \prime}$ & $0.10^{\prime \prime}$ & 4598.00 & 4208.58(9.19) \\
\hline E2-H-9 & & $3.00 "$ & & 3824.25 & \\
\hline
\end{tabular}


TABLE 10 (continued)

\begin{tabular}{|c|c|c|c|c|c|}
\hline Panel Type & Configurations & $\begin{array}{l}\text { Indenter/ } \\
\text { Impactor } \\
\text { Diameter }\end{array}$ & $\begin{array}{c}\text { Indentation } \\
\text { Depth/Impact } \\
\text { Energy }\end{array}$ & $\begin{array}{c}\text { CAI } \\
\text { Strength } \\
\text { (lb/in) }\end{array}$ & Avg.* $\left(\mathrm{CV}^{* *}\right)$ \\
\hline E2-H-10a & \multirow{3}{*}{ DNR } & \multirow{3}{*}{$3.00 "$} & \multirow{3}{*}{$0.15 "$} & 3377.25 & \multirow{3}{*}{$3326.75(2.48)$} \\
\hline E2-H-11 & & & & 3371.50 & \\
\hline E2-H-12 & & & & 3231.50 & \\
\hline E2-H-13 & \multirow{3}{*}{ DR } & \multirow{3}{*}{ 3.00" } & \multirow{3}{*}{$0.15 "$} & 3718.00 & \multirow{3}{*}{ 4067.08(8.82) } \\
\hline E2-H-14 & & & & 4048.75 & \\
\hline E2-H-15 & & & & 4434.50 & \\
\hline S1-FM94-P10-5 & \multirow{3}{*}{ UD } & \multirow{3}{*}{$1.00 "$} & \multirow{3}{*}{$40 \mathrm{in}-\mathrm{lb}$} & 1361.24 & \multirow{3}{*}{ 1368.28(1.10) } \\
\hline S1-FM94-P10-2 & & & & 1358.00 & \\
\hline S1-FM94-P10-5a & & & & 1385.60 & \\
\hline S1-FM94-P9-4 & \multirow{3}{*}{ DNR } & \multirow{3}{*}{$1.00 "$} & \multirow{3}{*}{$40 \mathrm{in}-\mathrm{lb}$} & 1197.75 & \multirow{3}{*}{ 1201.21(0.78) } \\
\hline S1-FM94-P10-2a & & & & 1194.08 & \\
\hline S1-FM94-P9-2a & & & & 1211.81 & \\
\hline S1-FM94-P11-3a & \multirow{3}{*}{$\mathrm{R}-1 \mathrm{H}$} & \multirow{3}{*}{$1.00 "$} & \multirow{3}{*}{$40 \mathrm{in}-\mathrm{lb}$} & 1355.04 & \multirow{3}{*}{ 1383.39(1.85) } \\
\hline S1-FM94-P9-2 & & & & 1404.95 & \\
\hline S1-FM94-P11-2 & & & & 1390.20 & \\
\hline S1-FM94-P11-1 & \multirow{3}{*}{$\mathrm{R}-2 \mathrm{H}$} & \multirow{3}{*}{$1.00 "$} & \multirow{3}{*}{$40 \mathrm{in}-\mathrm{lb}$} & 1251.27 & \multirow{3}{*}{ 1262.31(0.92) } \\
\hline S1-FM94-P11-3 & & & & 1274.42 & \\
\hline S1-FM94-P9-1 & & & & 1261.24 & \\
\hline S1-FM94-P11-1a & \multirow{3}{*}{$\mathrm{R}-3 \mathrm{H}$} & \multirow{3}{*}{$1.00 "$} & \multirow{3}{*}{$40 \mathrm{in}-\mathrm{lb}$} & 1413.85 & \multirow{3}{*}{$1432.46(1.28)$} \\
\hline S1-FM94-P10-3 & & & & 1450.64 & \\
\hline S1-FM94-P9-1a & & & & 1432.90 & \\
\hline
\end{tabular}

* Average of 3 specimens

** Coefficient of variation (CV) in \%

UD: Undamaged

DNR: Damaged-No Repair

DR: Damaged-Repair

DINR: Destructive Inspection-No Repair

DIR: Destructive Inspection-Repair

R-1H: Repair-1 Hole, R-2H: Repair-2 Holes, R-3H: Repair-3 Holes 
TABLE 11

SUMMARY OF RESULTS: MAXIMUM RESIDUAL DENT DEPTH AND PLANAR DAMAGE AREA

\begin{tabular}{|c|c|c|c|c|c|}
\hline $\begin{array}{l}\text { Panel } \\
\text { Type }\end{array}$ & $\begin{array}{c}\text { Damage/ } \\
\text { Repair } \\
\text { Type }\end{array}$ & $\begin{array}{l}\text { Indenter } \\
\text { Diameter }\end{array}$ & $\begin{array}{c}\text { Indentation } \\
\text { /Impact Energy }\end{array}$ & $\begin{array}{l}\text { Max. Residual Dent } \\
\text { Depth (in) }\end{array}$ & $\begin{array}{c}\text { Planar } \\
\text { Damage Area } \\
\left(\text { in }^{2}\right)\end{array}$ \\
\hline \multirow{5}{*}{$\mathrm{CRF}$} & UD & N/A & N/A & N/A & N/A \\
\hline & DNR & \multirow{4}{*}{$3.00 "$} & \multirow{4}{*}{$0.15^{\prime \prime}$} & $0.021(26.44)$ & $1.85 *(15.82)^{* *}$ \\
\hline & DR & & & $0.017(19.98)$ & $1.88(8.39)$ \\
\hline & DINR & & & 0.018 & 1.578 \\
\hline & DIR & & & 0.015 & 1.745 \\
\hline \multirow{9}{*}{$\begin{array}{l}\text { S2- } \\
\text { FM94 }\end{array}$} & UD & N/A & N/A & N/A & N/A \\
\hline & DNR & \multirow{4}{*}{$3.00 "$} & \multirow{4}{*}{$0.10^{\prime \prime}$} & $0.048(7.39)$ & $1.79(3.02)$ \\
\hline & DR & & & $0.01(2.37)$ & $1.76(8.76)$ \\
\hline & DINR & & & 0.014 & 1.630 \\
\hline & DIR & & & 0.005 & 1.631 \\
\hline & DNR & \multirow{4}{*}{$3.00 "$} & \multirow{4}{*}{$0.15^{\prime \prime}$} & $0.03(1.14)$ & $3.47(1.38)$ \\
\hline & DR & & & $0.02(7.27)$ & $3.45(3.58)$ \\
\hline & DINR & & & 0.019 & 3.329 \\
\hline & DIR & & & 0.025 & 3.178 \\
\hline \multirow{9}{*}{ E2-H } & UD & N/A & N/A & N/A & N/A \\
\hline & DNR & \multirow{4}{*}{$3.00 "$} & \multirow{4}{*}{$0.10^{\prime \prime}$} & $0.01(3.15)$ & $1.93(2.12)$ \\
\hline & DR & & & $0.01(19.09)$ & 1.51(8.59) \\
\hline & DINR & & & 0.011 & 1.900 \\
\hline & DIR & & & 0.011 & 1.565 \\
\hline & DNR & \multirow{4}{*}{$3.00 "$} & \multirow{4}{*}{$0.15^{\prime \prime}$} & $0.02(4.58)$ & $3.29(5.78)$ \\
\hline & DR & & & $0.02(32.13)$ & $2.57(6.22)$ \\
\hline & DINR & & & 0.019 & 3.163 \\
\hline & DIR & & & 0.017 & 2.723 \\
\hline \multirow{5}{*}{$\begin{array}{l}\text { S1- } \\
\text { FM94 }\end{array}$} & UD & N/A & N/A & N/A & N/A \\
\hline & DNR & \multirow{4}{*}{$1.0^{\prime \prime}$} & \multirow{4}{*}{40 in-lb } & N/A & $1.39(11.76)$ \\
\hline & $\mathrm{R}-1 \mathrm{H}$ & & & N/A & $1.45(13.12)$ \\
\hline & $\mathrm{R}-2 \mathrm{H}$ & & & N/A & $1.40(6.71)$ \\
\hline & $\mathrm{R}-3 \mathrm{H}$ & & & N/A & $1.41(5.62)$ \\
\hline
\end{tabular}


TABLE 12

SUMMARY OF REPAIR EFFECTIVENESS OF DIFFERENT SANDWICH TYPES

\begin{tabular}{|c|c|c|c|c|c|}
\hline \multirow{2}{*}{ Specimen ID } & \multirow{2}{*}{ Configurations } & $\begin{array}{c}\text { CAI } \\
\text { Strength }\end{array}$ & $\begin{array}{c}\text { Avg. } \\
\text { Strength }\end{array}$ & \multirow{2}{*}{$\begin{array}{c}\text { Repair } \\
\text { Effectiveness }\end{array}$} & \multirow{2}{*}{$\begin{array}{c}\text { Strength } \\
\text { Improvement } \\
\text { due to repair } \\
(\%)\end{array}$} \\
\hline & & \multicolumn{2}{|c|}{ (lb/in) } & & \\
\hline CRF-1 & \multirow{3}{*}{ UD } & 936.21 & \multirow{3}{*}{888.07} & \multirow{9}{*}{0.97} & \multirow{9}{*}{99.81} \\
\hline CRF-2 & & 895.05 & & & \\
\hline CRF-3 & & 832.94 & & & \\
\hline CRF-4 & \multirow{3}{*}{ DNR } & 733.43 & \multirow{3}{*}{823.61} & & \\
\hline CRF-5 & & 836.40 & & & \\
\hline CRF-6 & & 901.00 & & & \\
\hline CRF-7 & \multirow{3}{*}{ DR } & 955.71 & \multirow{3}{*}{886.40} & & \\
\hline CRF-8 & & 781.38 & & & \\
\hline CRF-9 & & 922.11 & & & \\
\hline S2-FM94-20 & \multirow{3}{*}{ UD } & 3824.34 & \multirow{3}{*}{3728.66} & \multirow{9}{*}{0.65} & \multirow{9}{*}{94.27} \\
\hline S2-FM94-2 & & 3450.50 & & & \\
\hline S2-FM94-3 & & 3911.15 & & & \\
\hline S2-FM94-4 & \multirow{3}{*}{ DNR } & 3038.75 & \multirow{3}{*}{3120.33} & & \\
\hline S2-FM94-5 & & 3051.75 & & & \\
\hline S2-FM94-6 & & 3270.50 & & & \\
\hline S2-FM94-7 & \multirow{3}{*}{ DR } & 3516.75 & \multirow{3}{*}{3515.00} & & \\
\hline S2-FM94-8 & & 3515.00 & & & \\
\hline S2-FM94-9 & & 3371.25 & & & \\
\hline S2-FM94-10 & \multirow{3}{*}{ DNR } & 1648.00 & \multirow{3}{*}{2346.75} & \multirow{6}{*}{0.67} & \multirow{6}{*}{87.86} \\
\hline S2-FM94-11 & & 2687.75 & & & \\
\hline S2-FM94-12 & & 2704.50 & & & \\
\hline S2-FM94-13 & \multirow{3}{*}{ DR } & 3282.00 & \multirow{3}{*}{3276.00} & & \\
\hline S2-FM94-14 & & 3437.25 & & & \\
\hline S2-FM94-15 & & 3108.75 & & & \\
\hline E2-H-1 & \multirow{3}{*}{ UD } & 4601.68 & \multirow{3}{*}{4562.57} & & \\
\hline E2-H-2 & & 4438.11 & & & \\
\hline E2-H-3 & & 4647.93 & & & \\
\hline E2-H-4 & & 4151.25 & & & \\
\hline E2-H-5 & DNR & 3559.25 & 3843.92 & 0.51 & 92.24 \\
\hline E2-H-6 & & 3821.25 & & & \\
\hline E2-H-7 & & 4203.50 & & & \\
\hline E2-H-8 & DR & 4598.00 & 4208.58 & & \\
\hline E2-H-9 & & 3824.25 & & & \\
\hline
\end{tabular}


TABLE 12 (continued)

\begin{tabular}{|c|c|c|c|c|c|}
\hline Specimen ID & Configurations & $\begin{array}{c}\text { CAI } \\
\text { Strength }\end{array}$ & $\begin{array}{c}\text { Avg. } \\
\text { Strength }\end{array}$ & $\begin{array}{c}\text { Repair } \\
\text { Effectiveness }\end{array}$ & $\begin{array}{c}\text { Strength } \\
\text { Improvement } \\
\text { due to repair } \\
(\%)\end{array}$ \\
\hline E2-H-10a & \multirow{3}{*}{ DNR } & 3377.25 & \multirow{3}{*}{3326.75} & \multirow{6}{*}{0.60} & \multirow{6}{*}{89.14} \\
\hline E2-H-11 & & 3371.50 & & & \\
\hline E2-H-12 & & 3231.50 & & & \\
\hline E2-H-13 & \multirow{3}{*}{ DR } & 3718.00 & \multirow{3}{*}{4067.08} & & \\
\hline E2-H-14 & & 4048.75 & & & \\
\hline E2-H-15 & & 4434.50 & & & \\
\hline S1-FM94-P10-5 & \multirow{3}{*}{ UD } & 1361.24 & \multirow{3}{*}{1368.28} & \multirow{9}{*}{1.09} & \multirow{9}{*}{101.10} \\
\hline S1-FM94-P10-2 & & 1358.00 & & & \\
\hline S1-FM94-P10-5a & & 1385.60 & & & \\
\hline S1-FM94-P9-4 & \multirow{3}{*}{ DNR } & 1197.75 & \multirow{3}{*}{1201.21} & & \\
\hline S1-FM94-P10-2a & & 1194.08 & & & \\
\hline S1-FM94-P9-2a & & 1211.81 & & & \\
\hline S1-FM94-P11-3a & \multirow{3}{*}{$\mathrm{R}-1 \mathrm{H}$} & 1355.04 & \multirow{3}{*}{1383.39} & & \\
\hline S1-FM94-P9-2 & & 1404.95 & & & \\
\hline S1-FM94-P11-2 & & 1390.20 & & & \\
\hline S1-FM94-P11-1 & \multirow{3}{*}{$\mathrm{R}-2 \mathrm{H}$} & 1251.27 & \multirow{3}{*}{1262.31} & \multirow{3}{*}{0.37} & \multirow{3}{*}{92.26} \\
\hline S1-FM94-P11-3 & & 1274.42 & & & \\
\hline S1-FM94-P9-1 & & 1261.24 & & & \\
\hline S1-FM94-P11-1a & \multirow{3}{*}{ R-3H } & 1413.85 & \multirow{3}{*}{1432.46} & \multirow{3}{*}{1.38} & \multirow{3}{*}{104.69} \\
\hline S1-FM94-P10-3 & & 1450.64 & & & \\
\hline S1-FM94-P9-1a & & 1432.90 & & & \\
\hline
\end{tabular}

\subsection{Failure Modes}

It can be observed that the in-plane compressive strength changes from undamaged to damaged specimens are noticeable. The resin repaired configuration was observed to restore/exceed the strength to the undamaged value in case of S1-FM94 specimens. The facesheets fracture which was observed to emanate from the center of the damage region is found to move away from damage region for the repaired specimens as shown in Figures 64 and 65 due to the presence of resin-repaired holes. The fracture locations of the undamaged and repaired specimens are observed to be similar. The failure modes of undamaged configuration 
are emanated away from the center of the specimens due to the absence of damage on the facesheet leading to either top or bottom end crushing failure modes. The onset of crack propagation on damaged configuration, through the center of the damaged zone resulted in a subsequent failure of specimens under in-plane compressive loading. Figure 63 illustrates the damage growth mechanism and final failure sequence observed in CRF, S2-FM94 and E2-H sandwich specimens with subsurface core damage. Figure 65 compares the final failure resulting from the facesheets fractures for undamaged, damaged and repaired configurations of CRF, S2FM94 and E2-H specimens. The repaired specimens were observed to be more effective in increasing the residual strength of the specimens. 


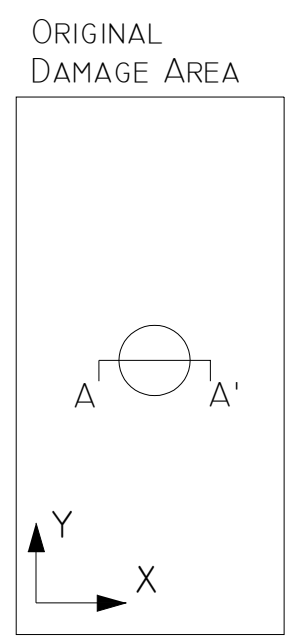

INCREASE IN

DIMPLE DEPTH

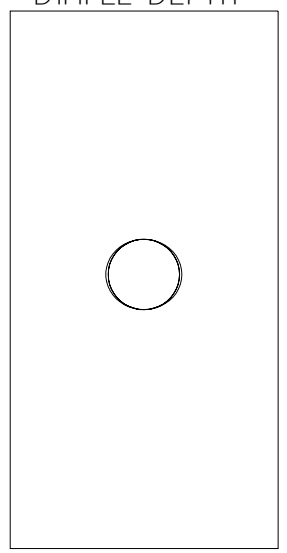

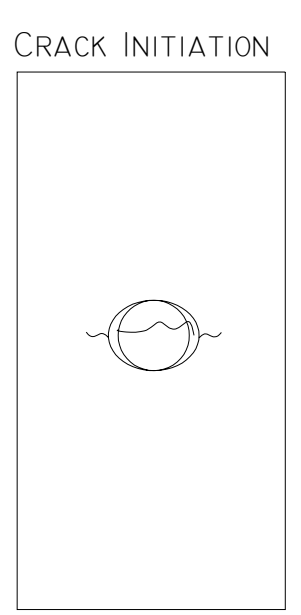

CRACK PROPAGATION

-FINAL FAILURE

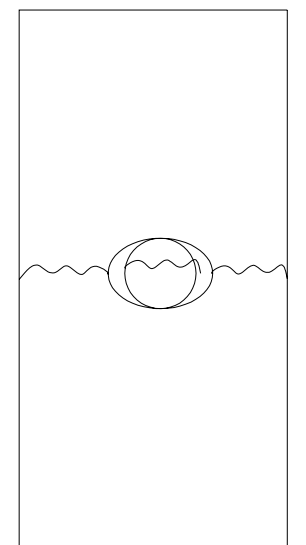

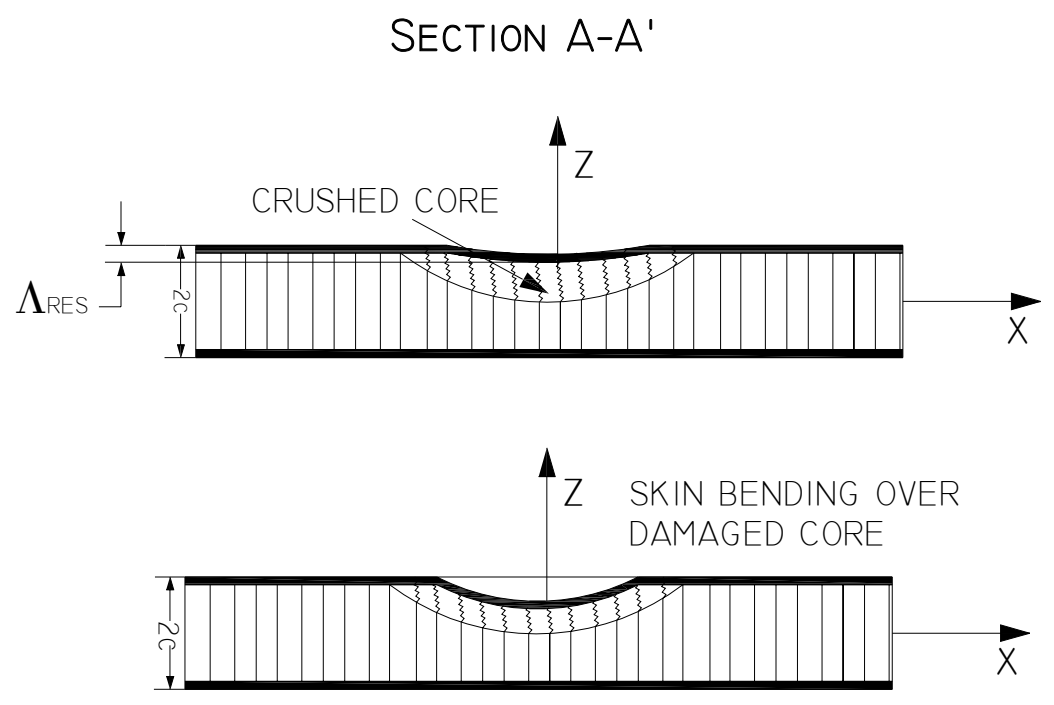

Figure 63: Damage growth mechanism and final failure mode observed in damaged CRF and S2FM94 sandwich specimens [3] 


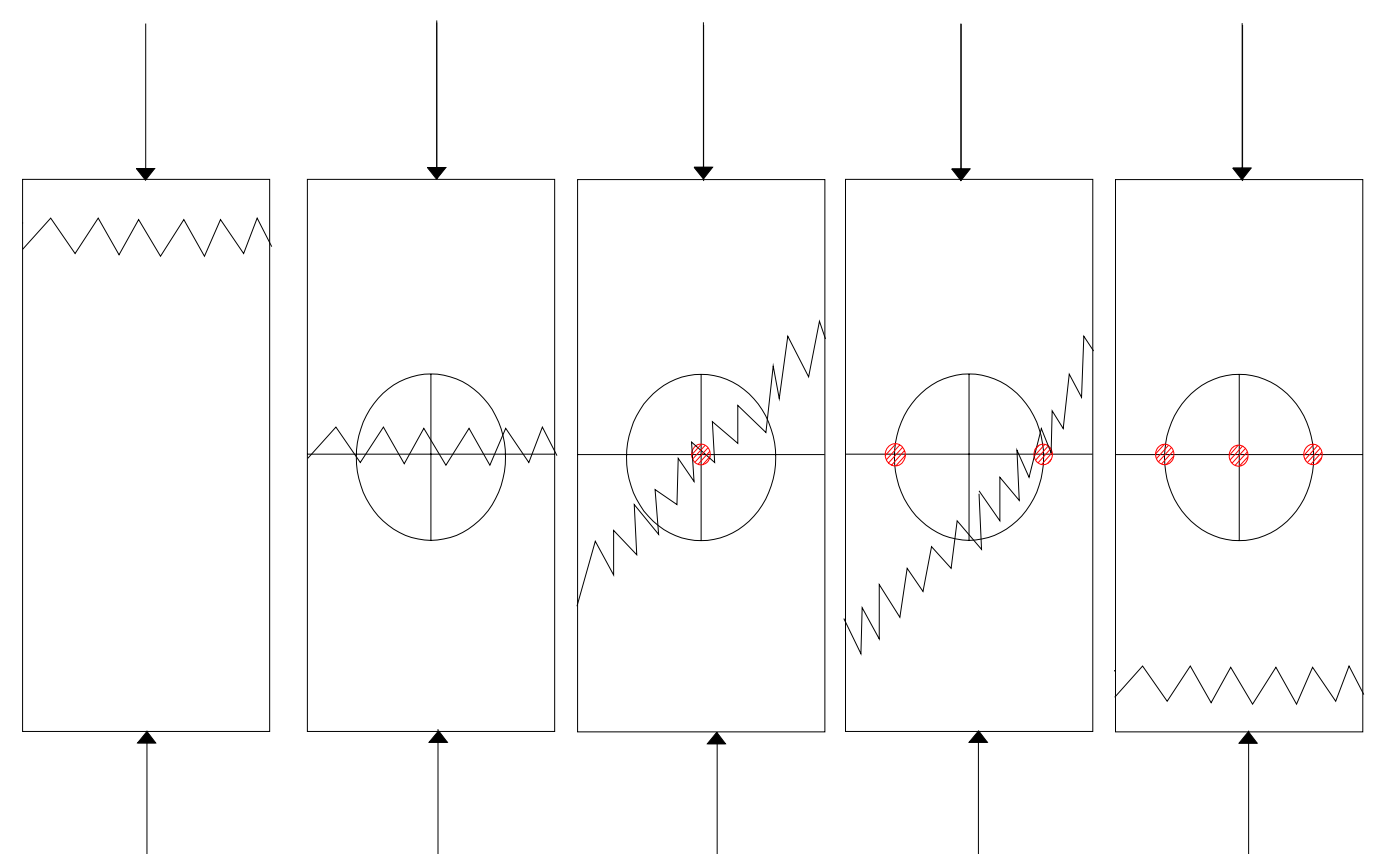

No Damage Impact Damage Impact-1 Hole Impact-2 Holes Impact-3 Holes

Figure 64: Different failure modes of specimens with varying number of holes

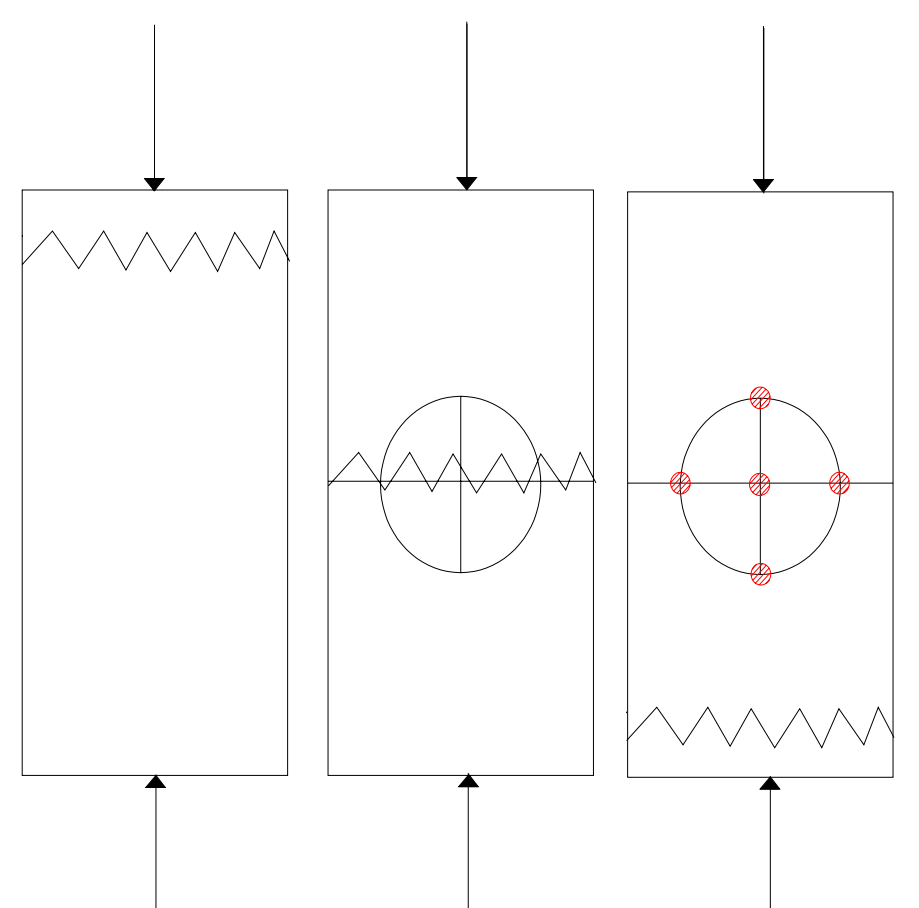

\section{Undamaged Damaged Damaged-Repair}

Figure 65: Final failure modes observed in CRF, S2-FM94 and E2-H sandwich panels for undamaged, damaged and repaired configurations 


\section{CHAPTER 6}

\section{CONCLUSIONS AND RECOMMENDATIONS}

\subsection{Conclusions}

Based on the experimental results, it is concluded that using a five-hole repair configuration, the residual strength can be restored close to the undamaged value. Compared to the experimental results obtained from S1-FM94 compression tests, conducted to evaluate the total number of holes for repair purposes and the locations of the holes, the fracture location was found to be moved away from the damaged region to a far field location; a $45^{\circ}$ crack growing across the damaged area was replaced with a horizontal crack propagating along the width of the specimen, located far from the repaired area as illustrated in Figure 64. In case of one-hole and two-hole repair configurations of S1-FM94, localized support was improved but was not enough to contain failure initiation in the damaged area. One-hole and two-hole repair configurations changed the nature of the load transfer across the damaged area propagating a crack in an inclined manner. The failure mode seen on repair configuration of CRF, S2-FM94 and E2-H specimens was identical to the facesheet fractures as seen on three-hole repair configuration of S1-FM94 specimens. It is evident that the presence of two additional repaired holes to provide strength enhancement along two orthogonal directions in the plane of the specimen does not change the fracture location compared to results observed in the case of three-hole repaired specimen, i.e., the fracture locations for three-hole repaired specimens and the five-hole repaired sandwich panels are identical. The failure modes for in-plane compression tests are visible along the width of the specimen. This shows that the occurrence of two repaired holes along the longitudinal axis of the sandwich specimens is not significant. The residual strength of damaged sandwich panels is improved by introducing resin repaired holes along perpendicular planes to 
the loading direction. Thus, the resin repair method can be used as an effective repair method for the containment of local bending in sandwich panels with subsurface core damage.

\subsection{Recommendations}

Based on the results of this investigation, the following are recommended for future work.

1. The effectiveness of repair for other loading scenarios and environmental conditions must be investigated to make the repair method suitable for practical use.

2. Repair using expandable foam must be investigated, due to its potential for accelerated field -repairs.

3. Develop an analysis tool (e.g., finite element model) to evaluate different cell-fill repair configurations and cell fill materials.

4. The digital speckle photography (DSP) Aramis [6] could be employed to record the deformations along $\mathrm{x}, \mathrm{y}$ and $\mathrm{z}$-directions for accurate data replacing strain gages and LVDT. 
REFERENCES 


\section{LIST OF REFERENCES}

[1] Composite Repair, Hexcel Corporate, Two Stamford Plaza, 16 ${ }^{\text {th }}$ Floor, 281 Tresser Boulevard, Stamford, Connecticut 06901, USA, Tel +1 (203) 969 0666, Fax +1 (203) 3583977

[2] DIAB Sandwich Handbook, DIAB Inc., 315 Seahawk Drive, Desoto, TX 75115, USA Tel +1 (972) 228 7600, Fax +1 (972) 2282667

[3] Tomblin, J. S., Lacy, T., Raju, S. K., Acosta J. F., Smith B. L., and Romine N. A., "Impact Damage Characterization and Damage Tolerance of Composite Sandwich Airframe Structures - Phase II,” Final Report, DOT/FAA/AR-02/80, October 2002.

[4] Serge Abrate, “Impact on Composite Structures,” 1998, pp. 140, 152.

[5] J. Tomblin S., K. S., Raju, Liew J., and Smith B. L., "Impact Damage Characterization and Damage Tolerance of Composite Sandwich Airframe Structures,” Final Report, DOT/FAA/AR-00/44, January 2001

[6] Andrei Shipsha, and Dan Zenkert, "Compression-after-Impact Strength of Sandwich Panels with Core Crushing Damage,” Applied Composite Materials, DOI: 10.1007/s 10443-005 [1] 9-1, 2005, pp. 149, 164.

[7] M. Raju, C. Rajasekhar Reddy, M.R. Narasimha Swamy, G. Giridhar, L. Srikanth, M. Rajendra Prakash and R.M.V.G.K. Rao, "Repair Effectiveness Studies on Impact Damaged Sandwich Composite Constructions,” Journal of Reinforced Plastics and Composites, Vol. 25, No. 1, 2006, pp. 5, 15.

[8] John S. Tomblin, Lamia Salah, John M. Welch, and Michael D. Borgman, “Bonded Repair of Aircraft Composite Sandwich Structures,” Final Report, DOT/FAA/AR-03/74, February 2004.

[9] Nobuo Takeda, Shu Minakuchi, and Yoji Okabe, "Smart Composite Sandwich Structure for Future Aerospace Application-Damage Detection and Suppression-: a Review,” Journal of Solid Mechanics and Materials Engineering, Vol. 1, No. 1, 2007, pp. 1, 15.

[10] Cytec Engineered Materials Inc., 4300 Jackson Street, Greenville, Texas 75401

[11] Newport Adhesives and Composites Inc., 1822 Reynolds Ave., Irvine, California 926145680, USA, Tel +1 (949) 2535680

[12] Hexcel, 6400 West 5400 South Salt Lake City, Utah USA 84118

[13] URL:http://miller-stephenson.com/epoxy_resins.htm/ 
[14] Mohaupt, A. A., and Heebink, B. G., "Supplement to Repair of Aircraft Sandwich Constructions 1, 2,” Aircraft Design Criteria, Report No. 1584-A, May 1950, pp. 1, 6.

[15] RDP Electrosense, 2216 Pottstown Pike, Pottstown, PA 19465

[16] Vishay Intertechnology, Inc., 63 Lancaster Avenue, Malvern, PA 19355-2143

[17] Loctite Aerospace, 2850 Willow Pass Road, Bay Point, CA 94565-3237, USA Tel +1 (800) 4249300 
APPENDICES 


\section{APPENDIX A}

END SHORTENING RESPONSE OF S2-FM94, E2-H AND CRF SANDWICH SPECIMENS

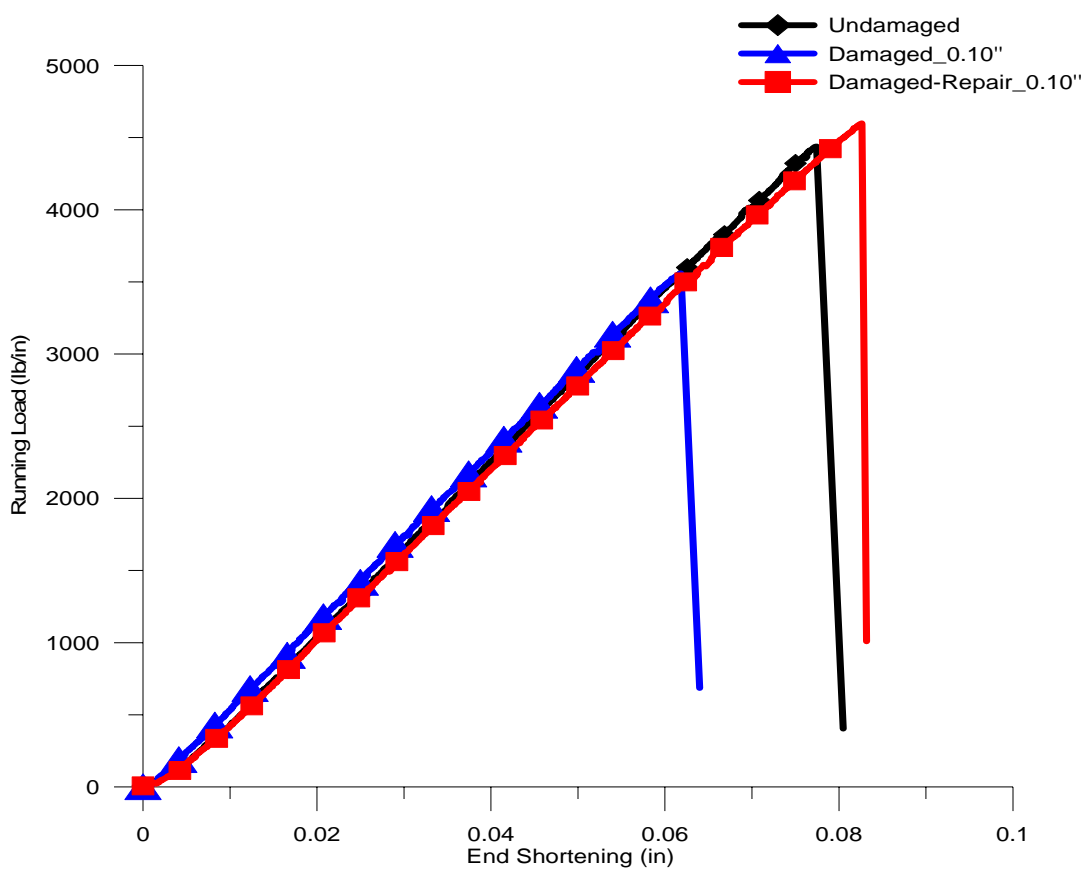

Figure 66: Running load versus end shortening of E2-H panels with indentation depth of 0.10"

$$
\begin{aligned}
& \text { Undamaged } \\
& \text { Damaged_0.10" } \\
& \text { Damaged-Repair_0.10" }
\end{aligned}
$$

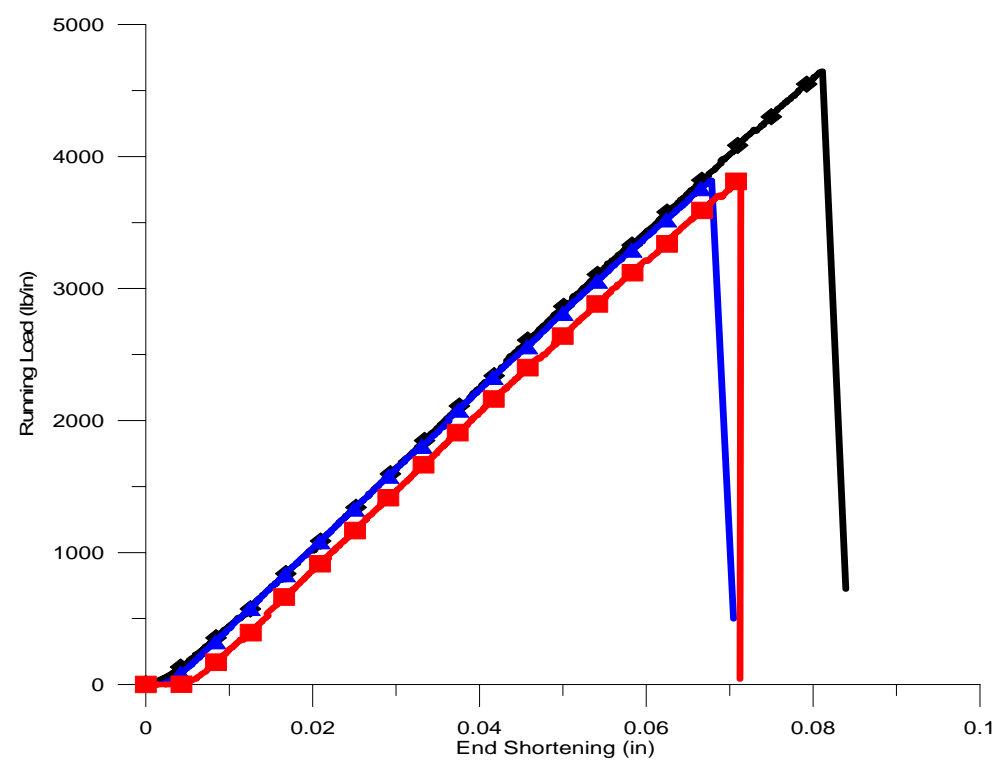

Figure 67: Running load versus end shortening of E2-H panels with indentation depth of 0.10" 


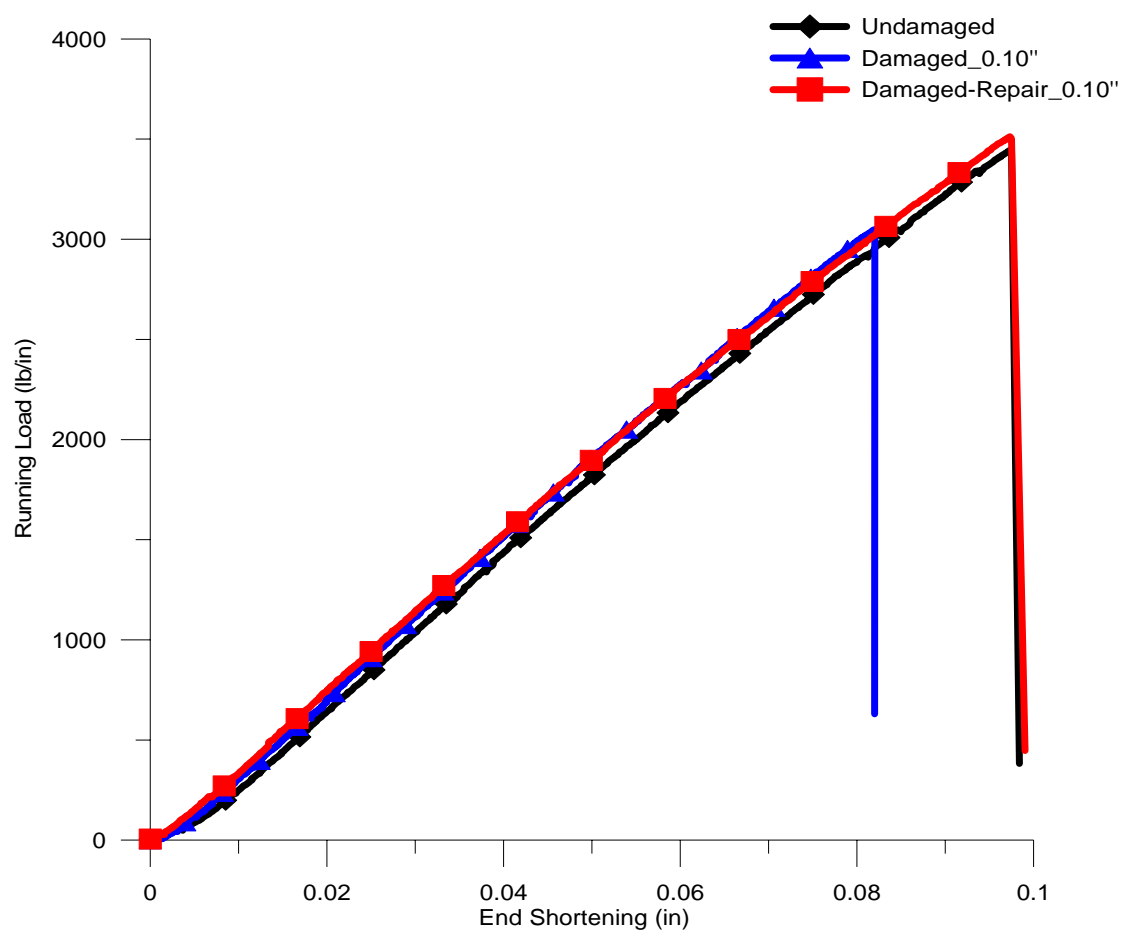

Figure 68: Running load versus end shortening of S2-FM94 panels with indentation depth of $0.10^{\prime \prime}$

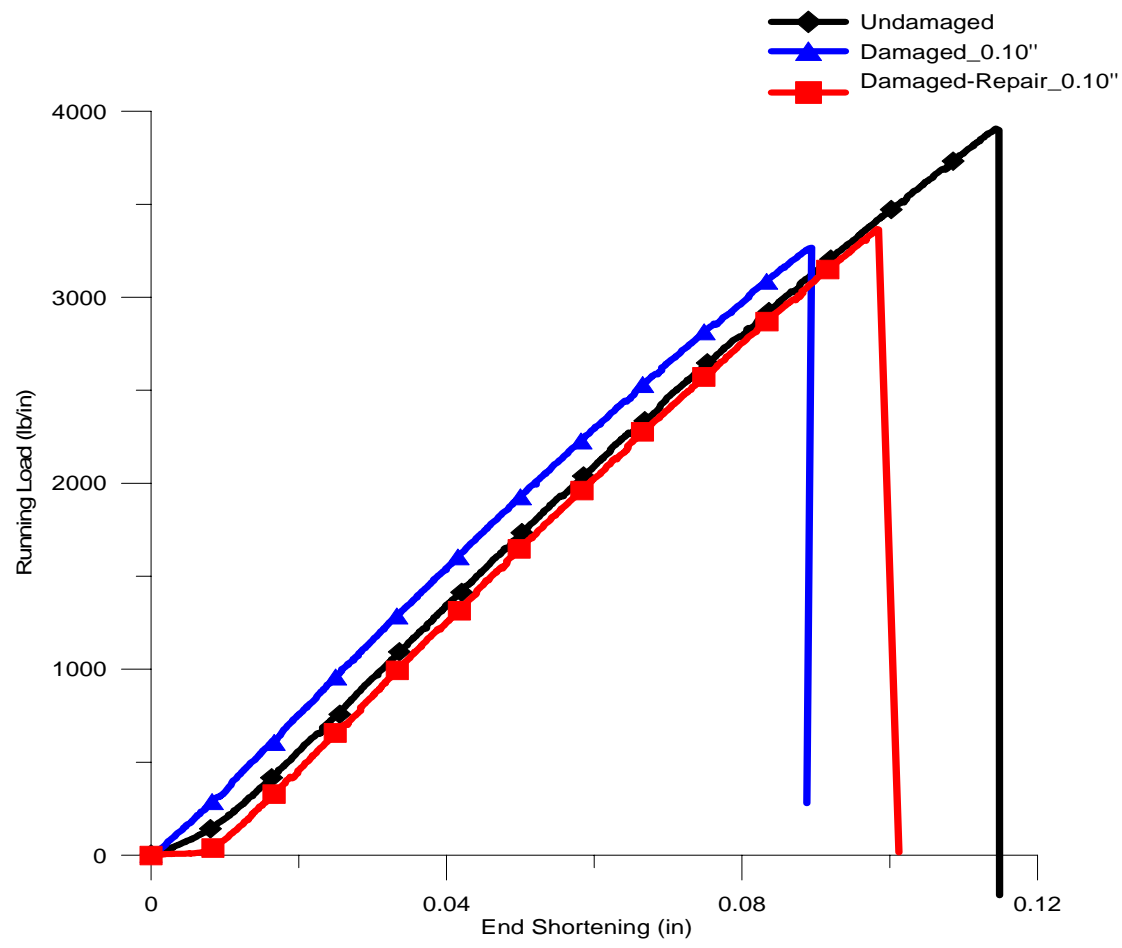

Figure 69: Running load versus end shortening of S2-FM94 panels with indentation depth of $0.10^{\prime \prime}$ 


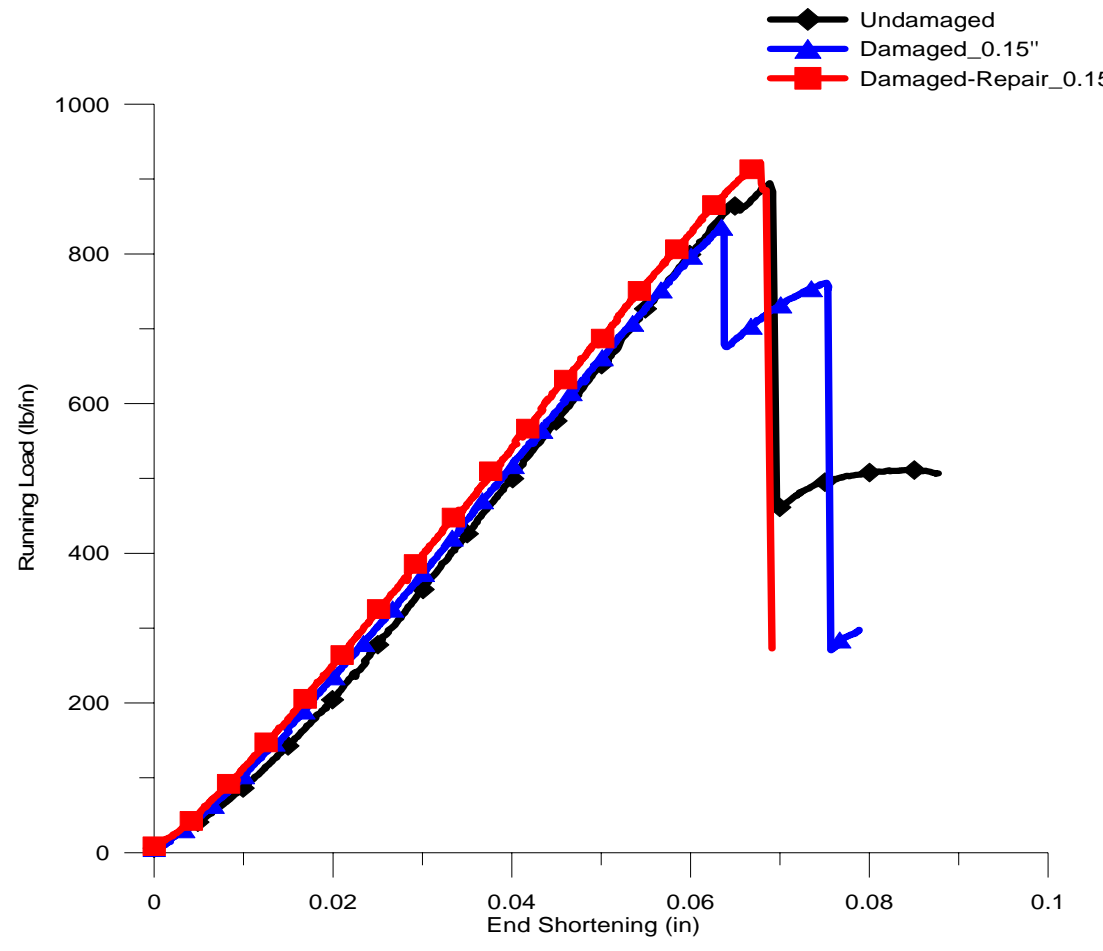

Figure 70: Running load versus end shortening of CRF panels with indentation depth of $0.15^{\prime \prime}$

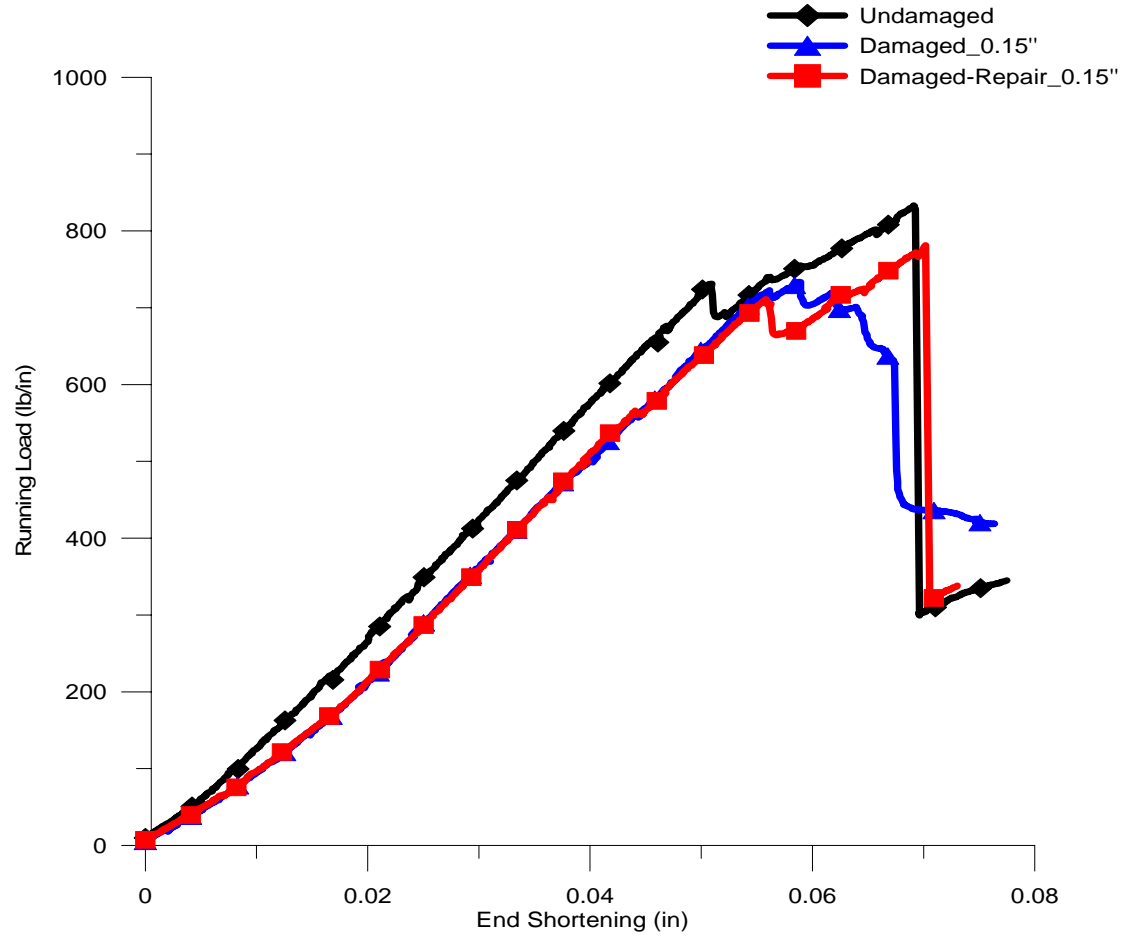

Figure 71: Running load versus end shortening of CRF panels with indentation depth of 0.15" 


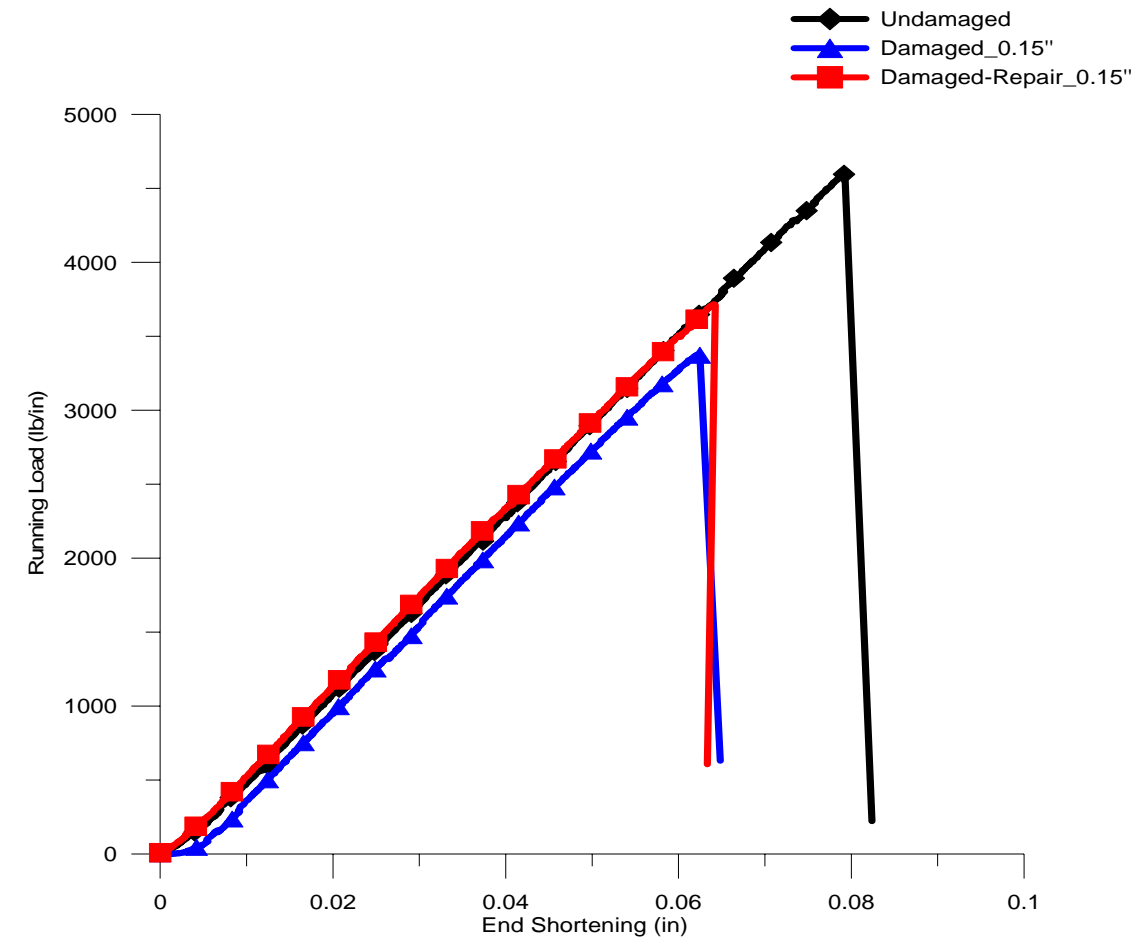

Figure 72: Running load versus end shortening of E2-H Sandwich

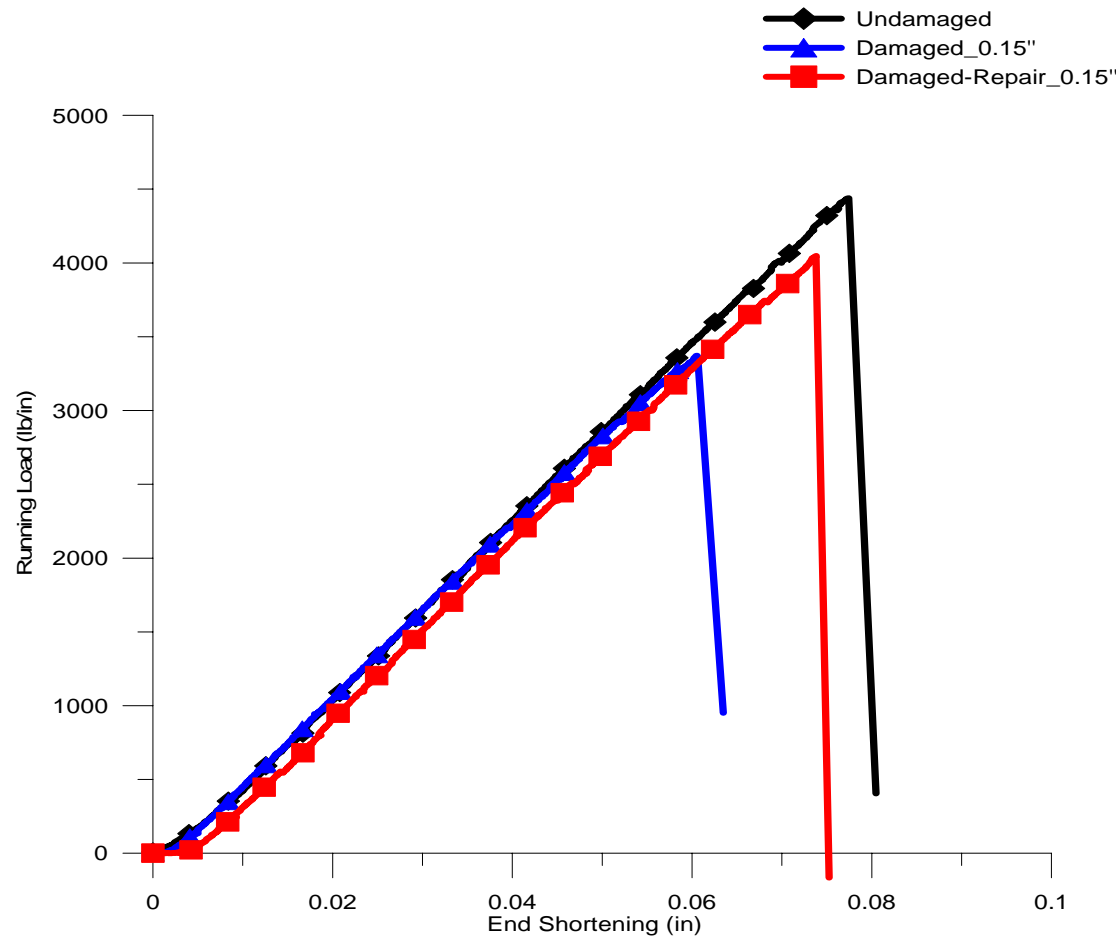

Figure 73: Running load versus end shortening of E2-H panels with indentation depth of 0.15" 

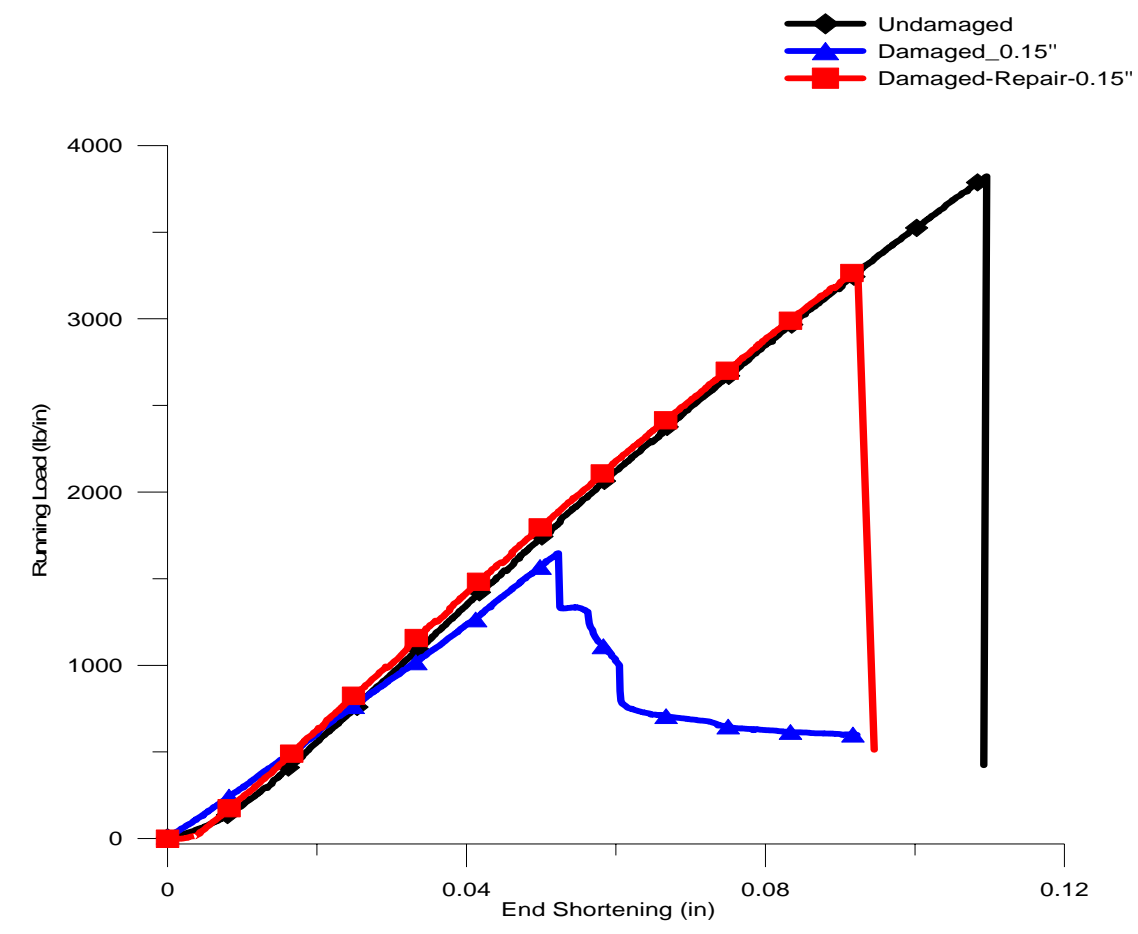

Figure 74: Running load versus end shortening of S2-FM94 Sandwich

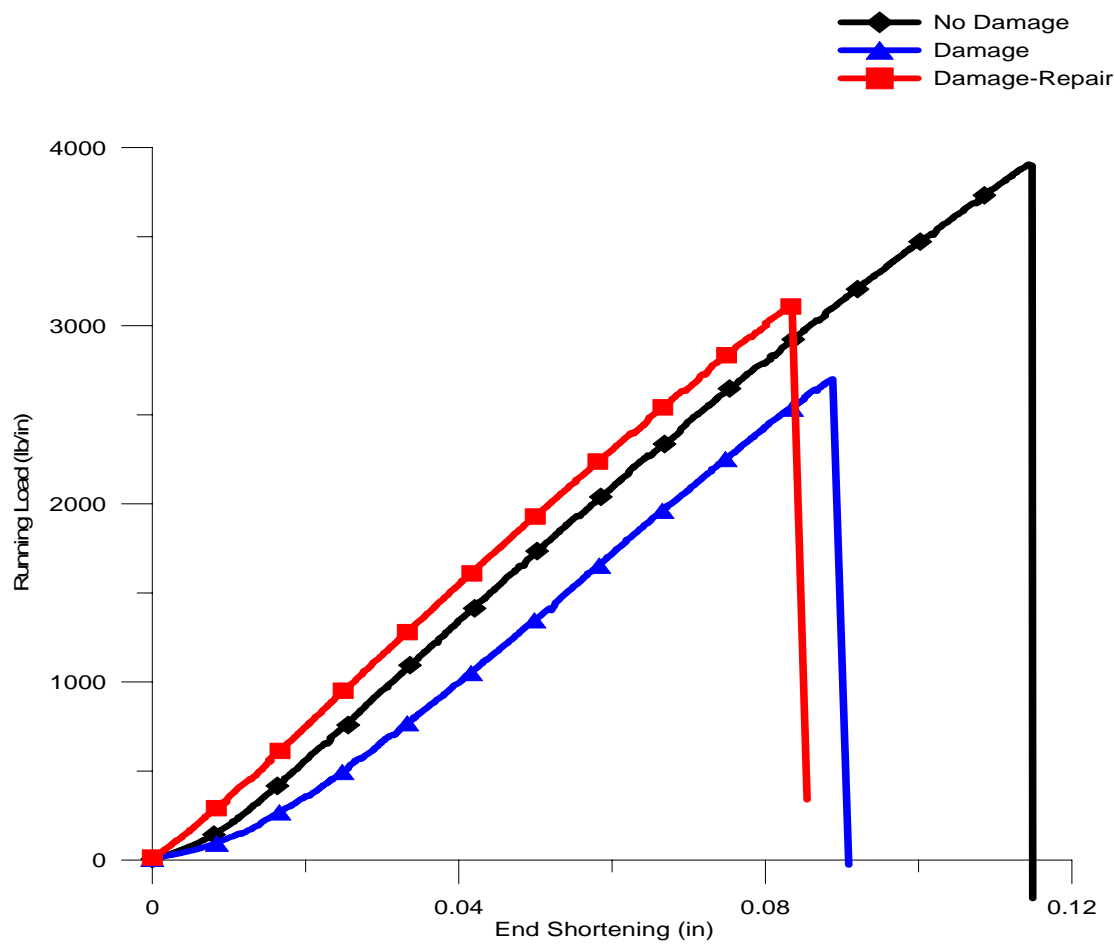

Figure 75: Running load versus end shortening of S2-FM94 panels with indentation depth of $0.15^{\prime \prime}$ 


\section{APPENDIX B}

OUT-OF-PLANE DISPLACEMENT RESPONSE OF S2-FM94, E2-H AND CRF SANDWICH SPECIMENS

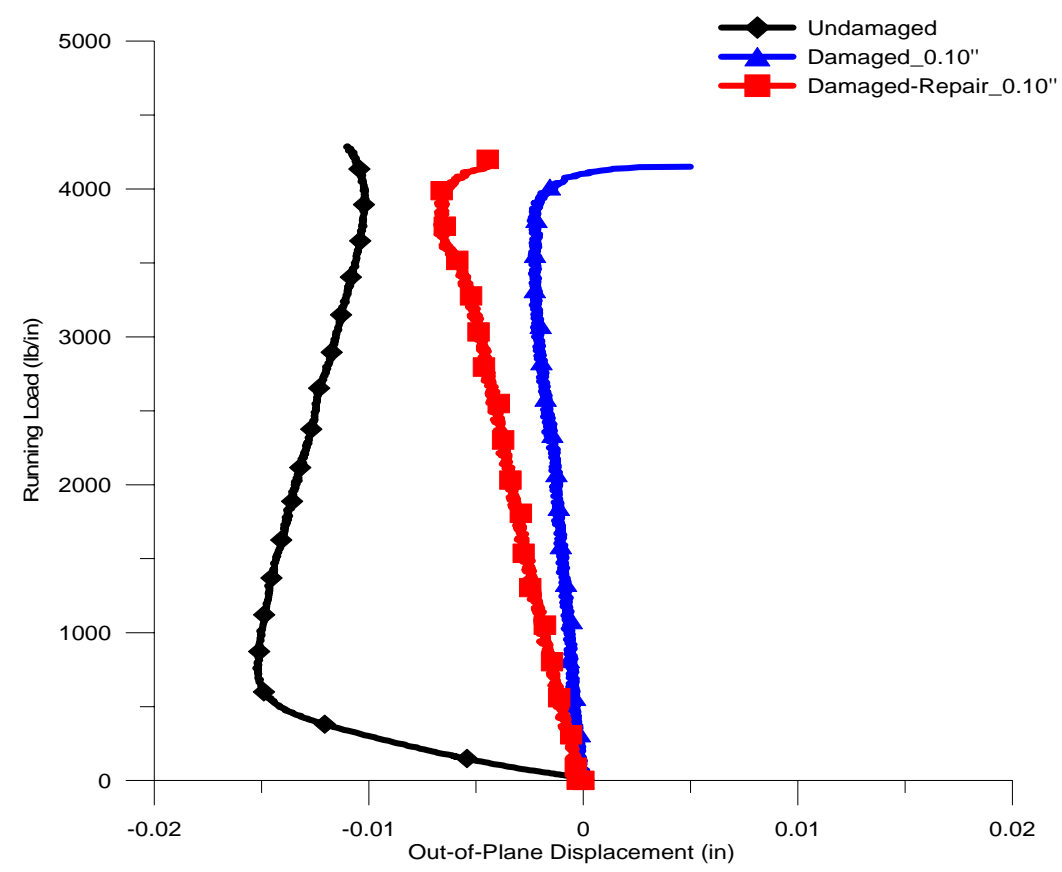

Figure 76: Running load versus out-of-plane displacement of E2-H panels with indentation depth

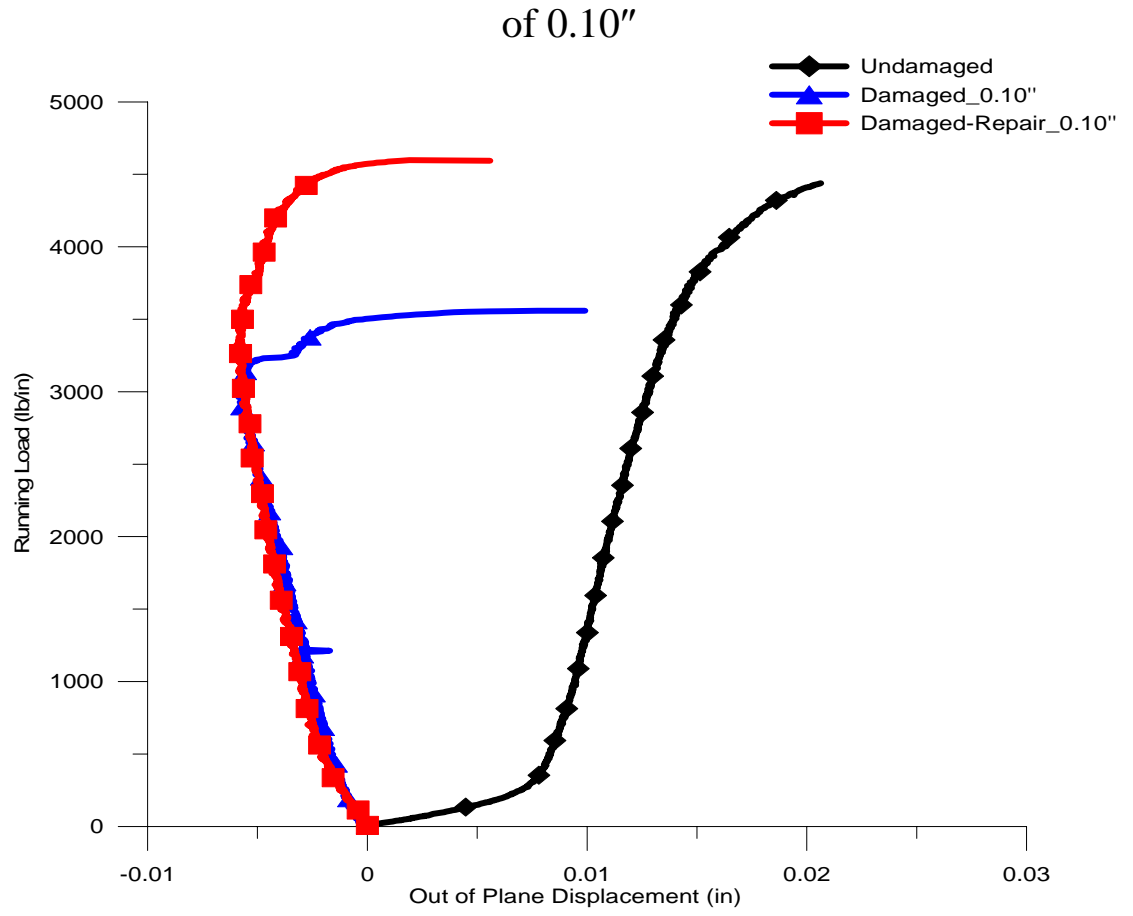

Figure 77: Running load versus out-of-plane displacement of E2-H panels with indentation depth of $0.10^{\prime \prime}$ 


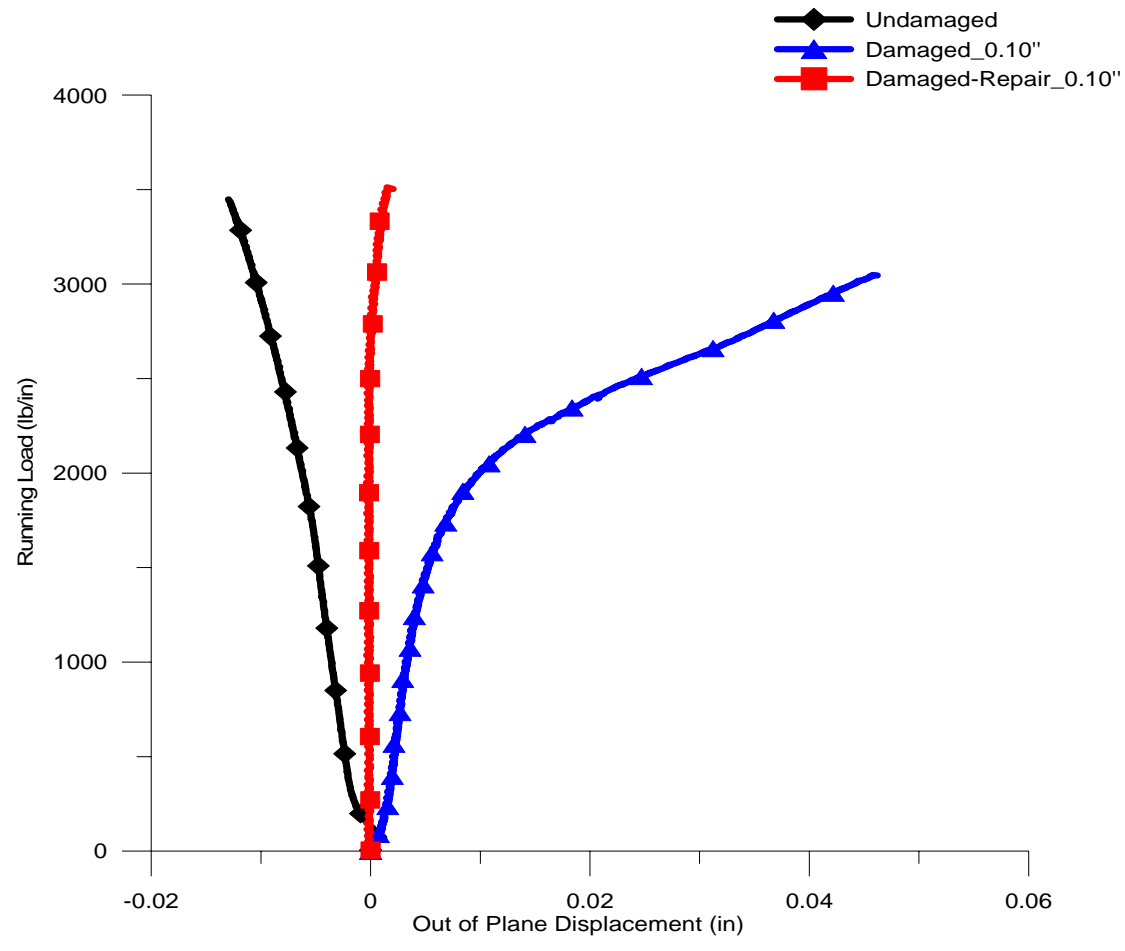

Figure 78: Running load versus out-of-plane displacement of S2-FM94 panels with indentation depth of $0.10^{\prime \prime}$
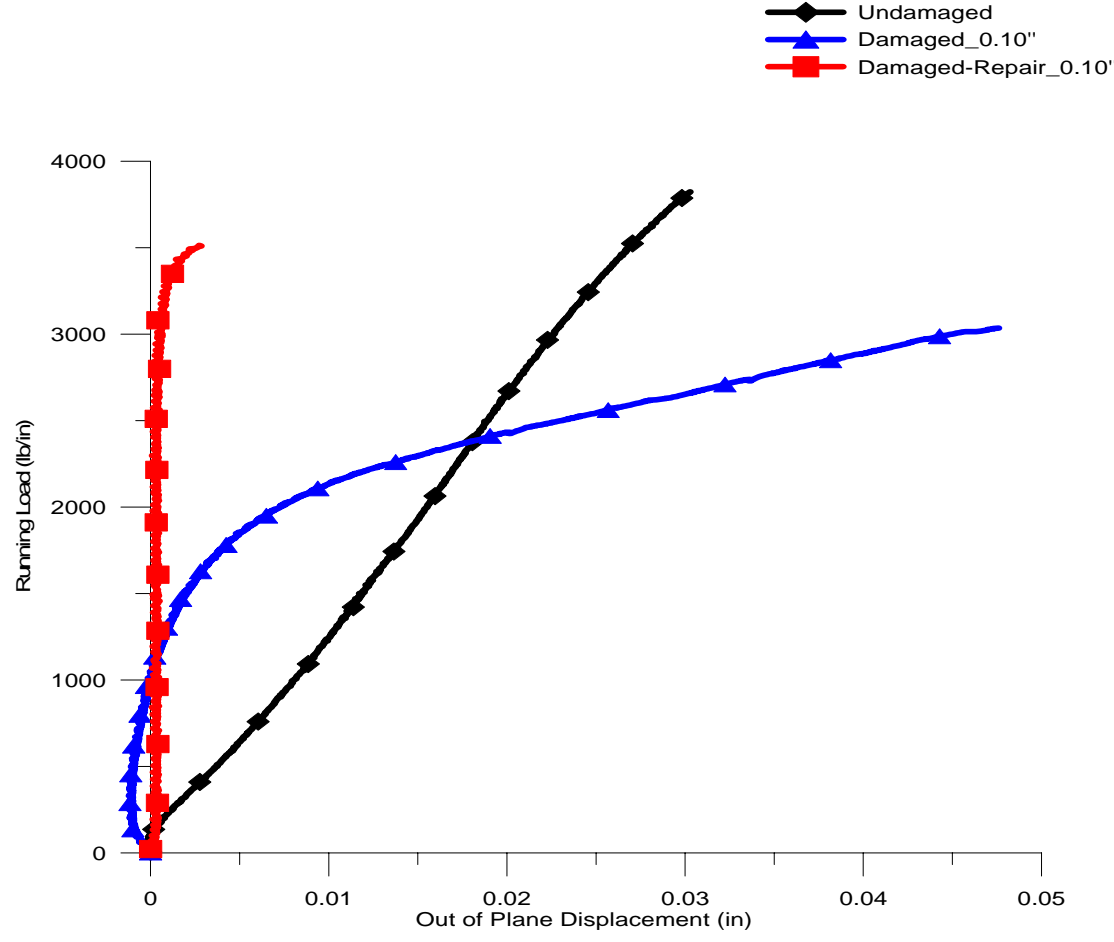

Figure 79: Running load versus out-of-plane displacement of S2-FM94 panels with indentation depth of $0.10^{\prime \prime}$ 


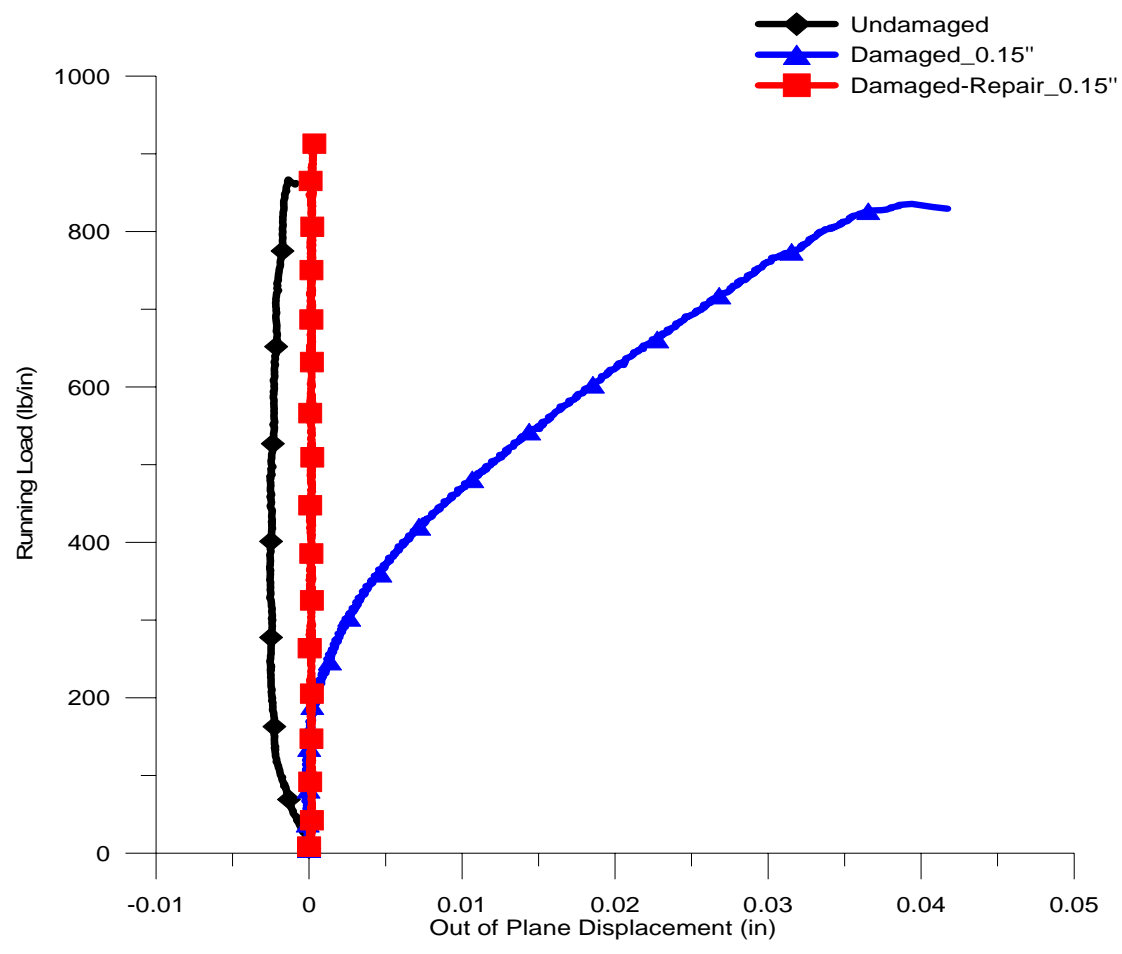

Figure 80: Running load versus out-of-plane displacement of CRF panels with indentation depth of $0.15^{\prime \prime}$

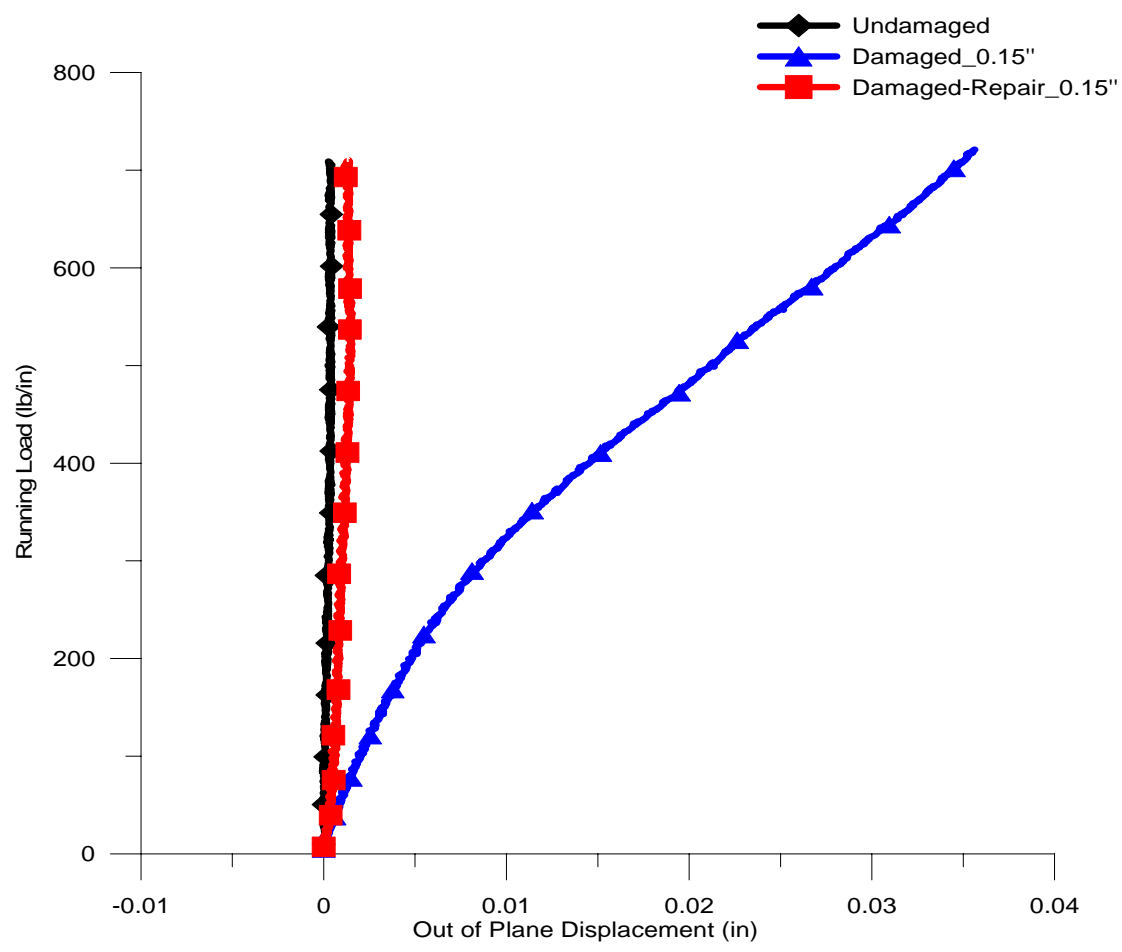

Figure 81: Running load versus out-of-plane displacement of CRF panels with indentation depth of $0.15^{\prime \prime}$ 


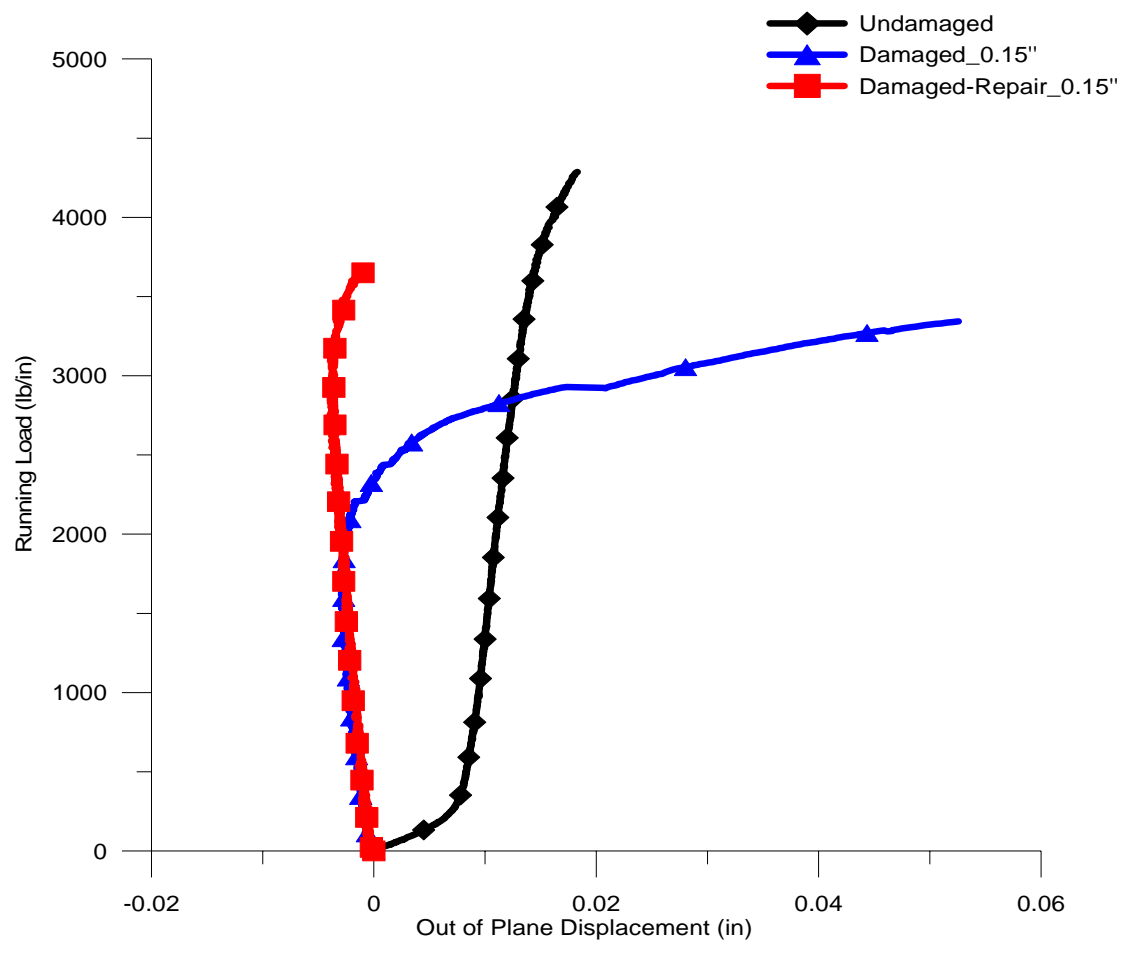

Figure 82: Running load versus out-of-plane displacement of E2-H panels with indentation depth of $0.15^{\prime \prime}$

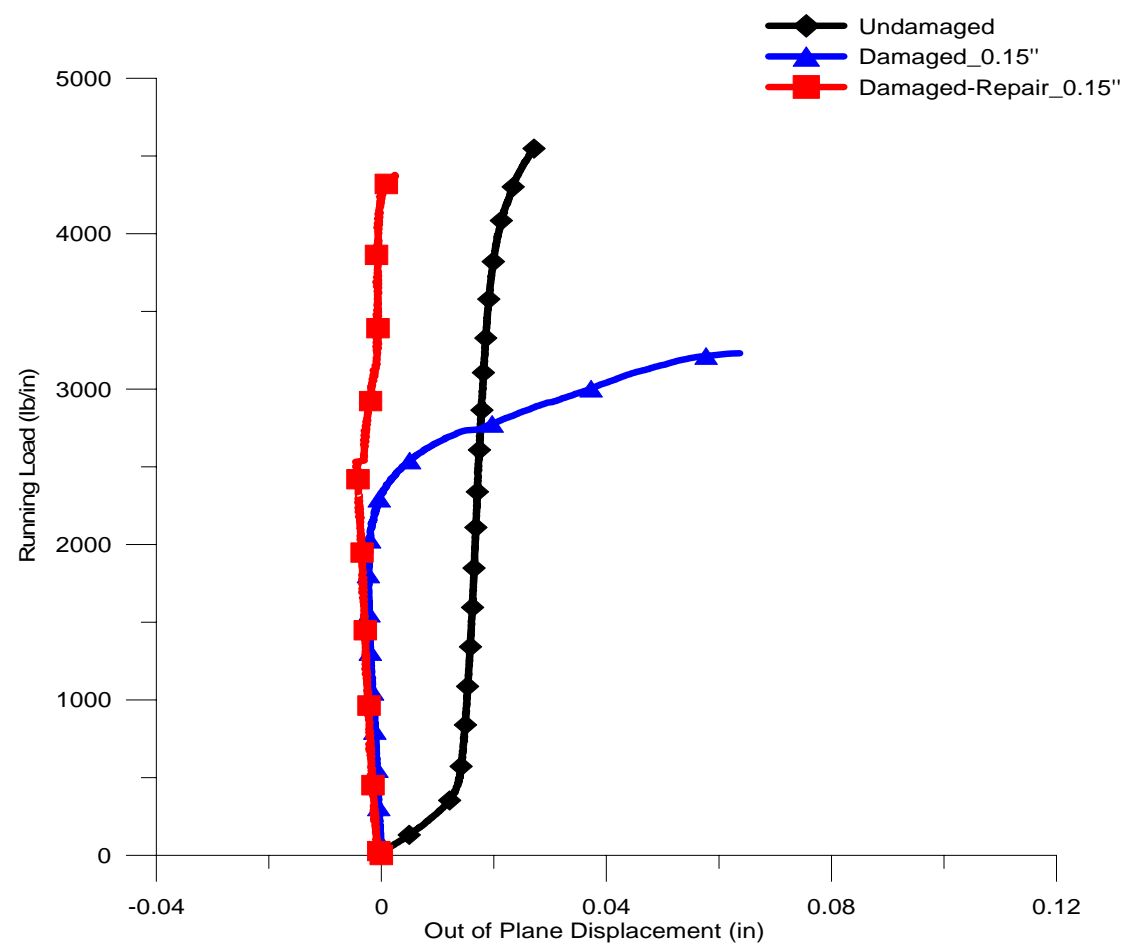

Figure 83: Running load versus out-of-plane displacement of E2-H panels with indentation depth of $0.15^{\prime \prime}$ 


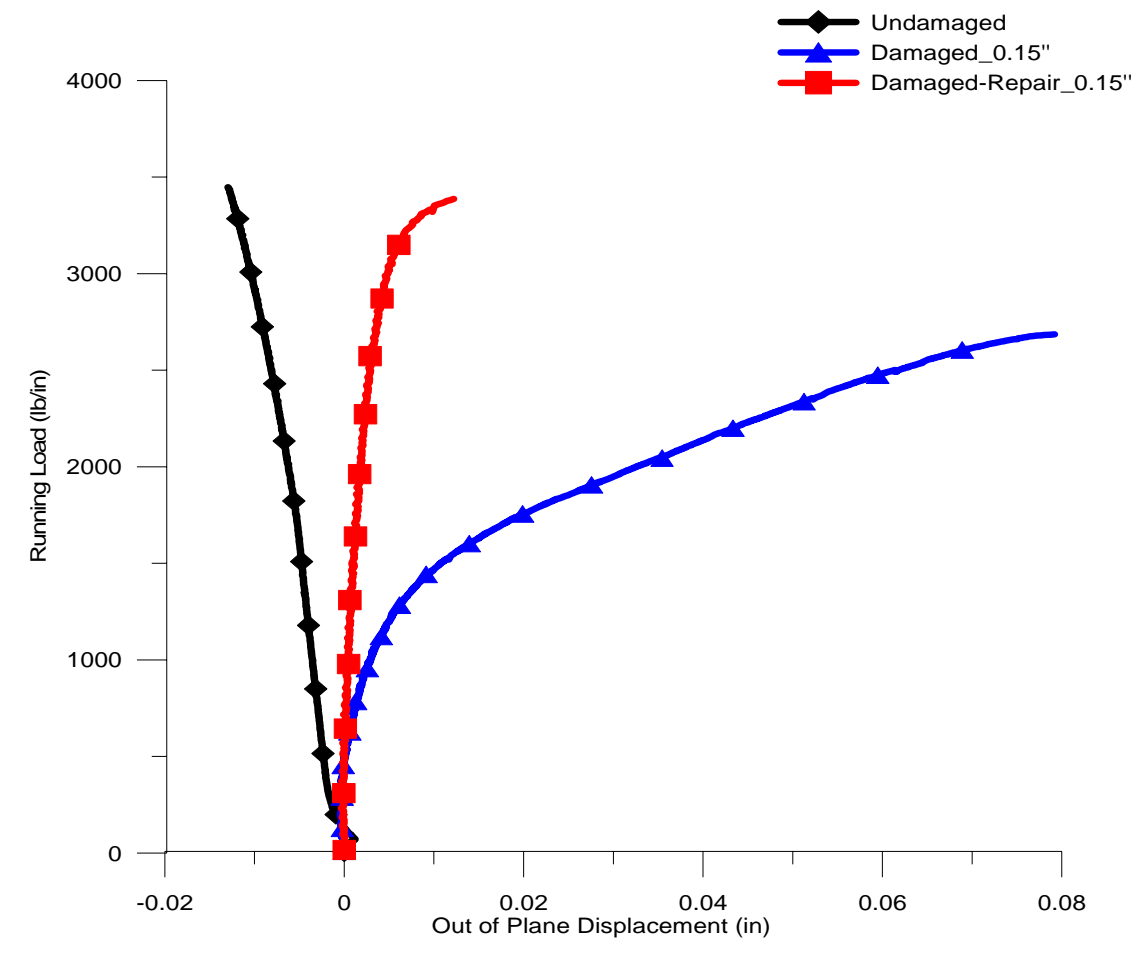

Figure 84: Running load versus out-of-plane displacement of S2-FM94 panels with indentation depth of $0.15^{\prime \prime}$

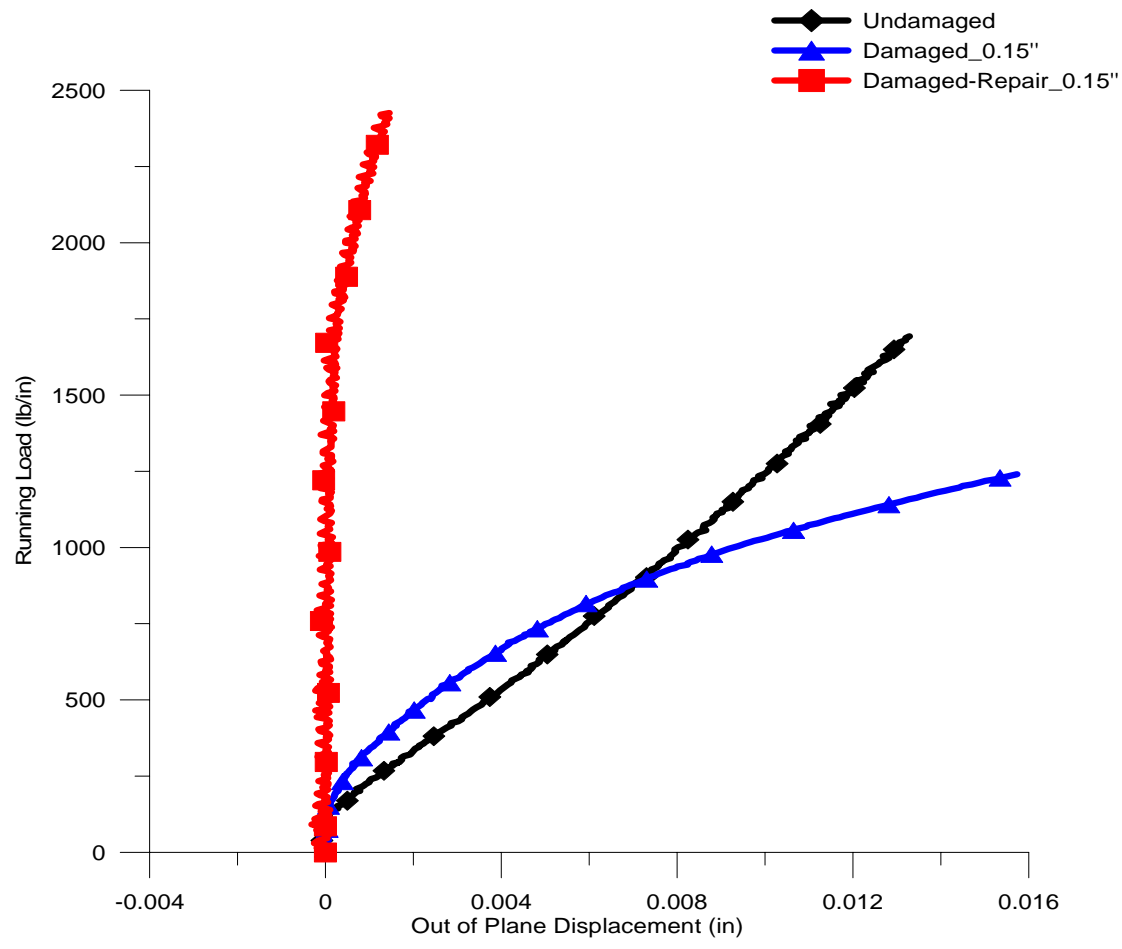

Figure 85: Running load versus out-of-plane displacement of S2-FM94 panels with indentation depth of $0.15^{\prime \prime}$ 


\section{APPENDIX C}

NONDESTRUCTIVE INSPECTIONS OF E2-H SANDWICH SPECIMENS WITH AND WITHOUT REPAIR

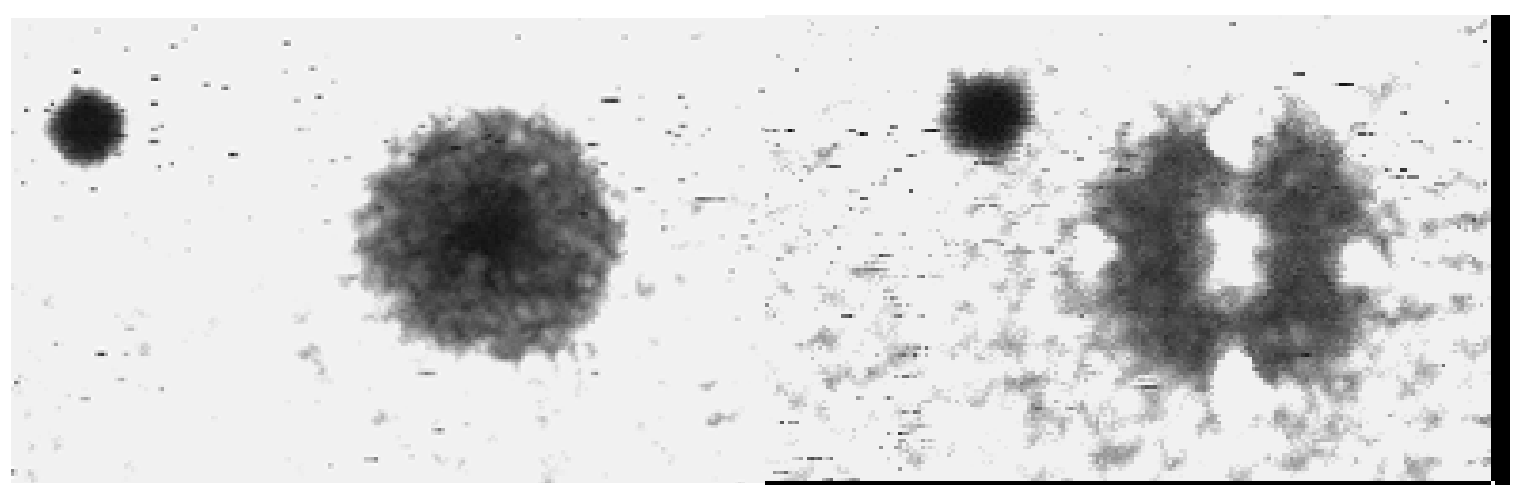

Figure 86: TTU C-scan image of E2-H-07 with (right) and without (left) 5-Hole Repair

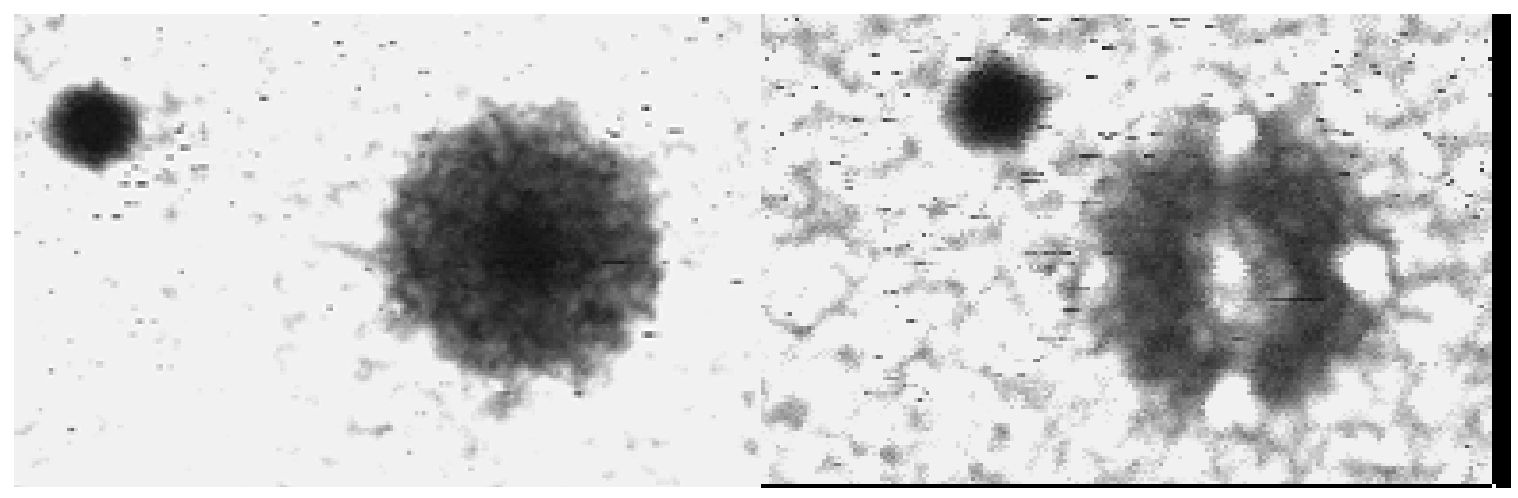

Figure 87: TTU C-scan image of E2-H-09 with (right) and without (left) 5-Hole Repair

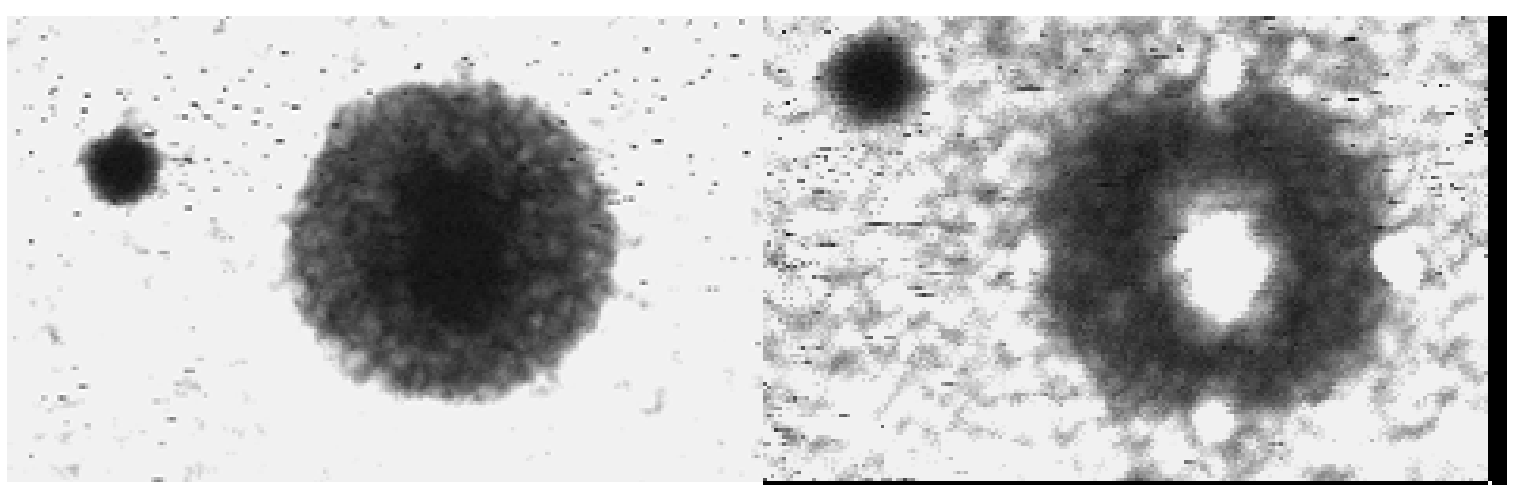

Figure 88: TTU C-scan image of E2-H-14 with (right) and without (left) 5-Hole Repair 


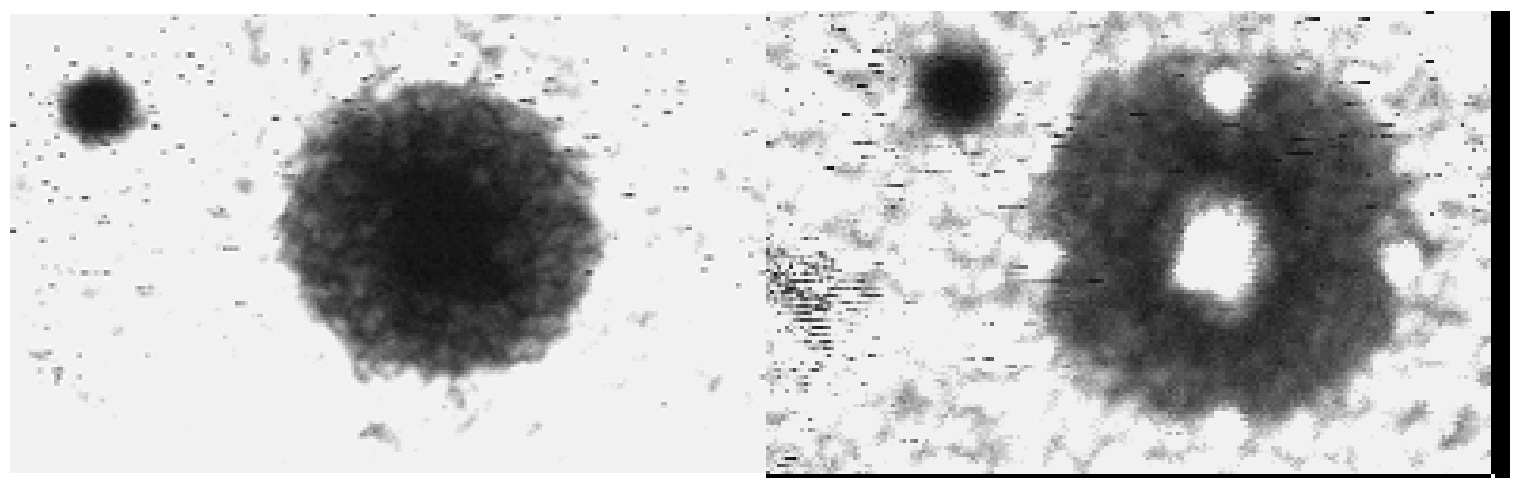

Figure 89: TTU C-scan image of E2-H-13 with (right) and without (left) 5-Hole Repair
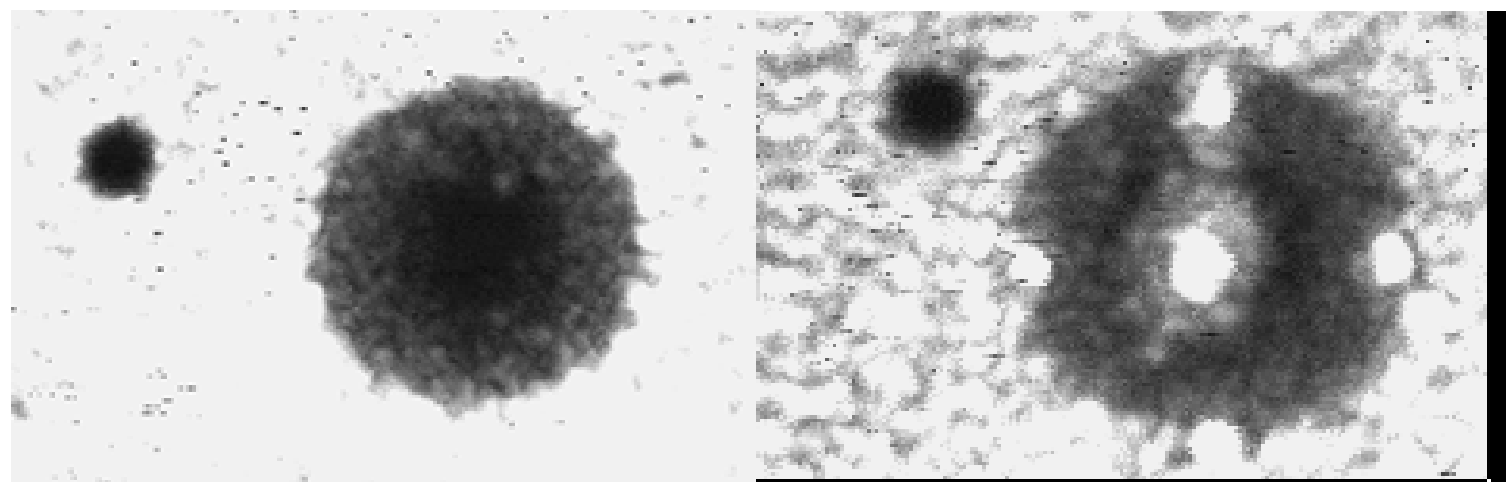

Figure 90: TTU C-scan image of E2-H-19 with (right) and without (left) 5-Hole Repair

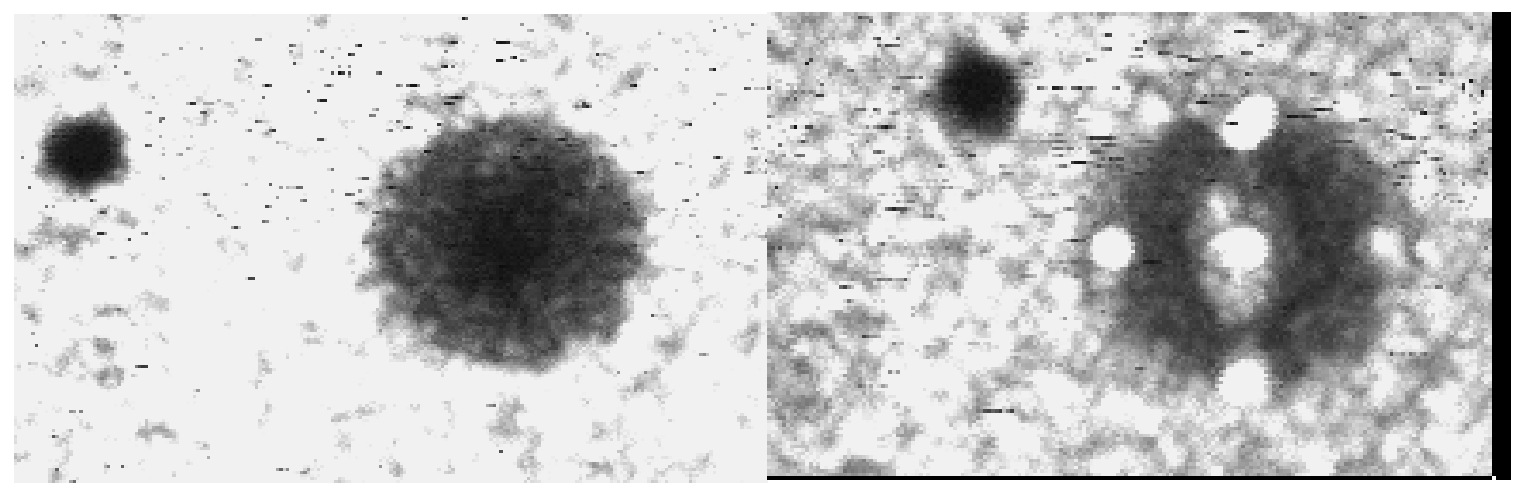

Figure 91: TTU C-scan image of E2-H-17 with (right) and without (left) 5-Hole Repair 


\section{APPENDIX D}

NONDESTRUCTIVE INSPECTIONS OF S2-FM94 SANDWICH SPECIMENS WITH AND WITHOUT REPAIR

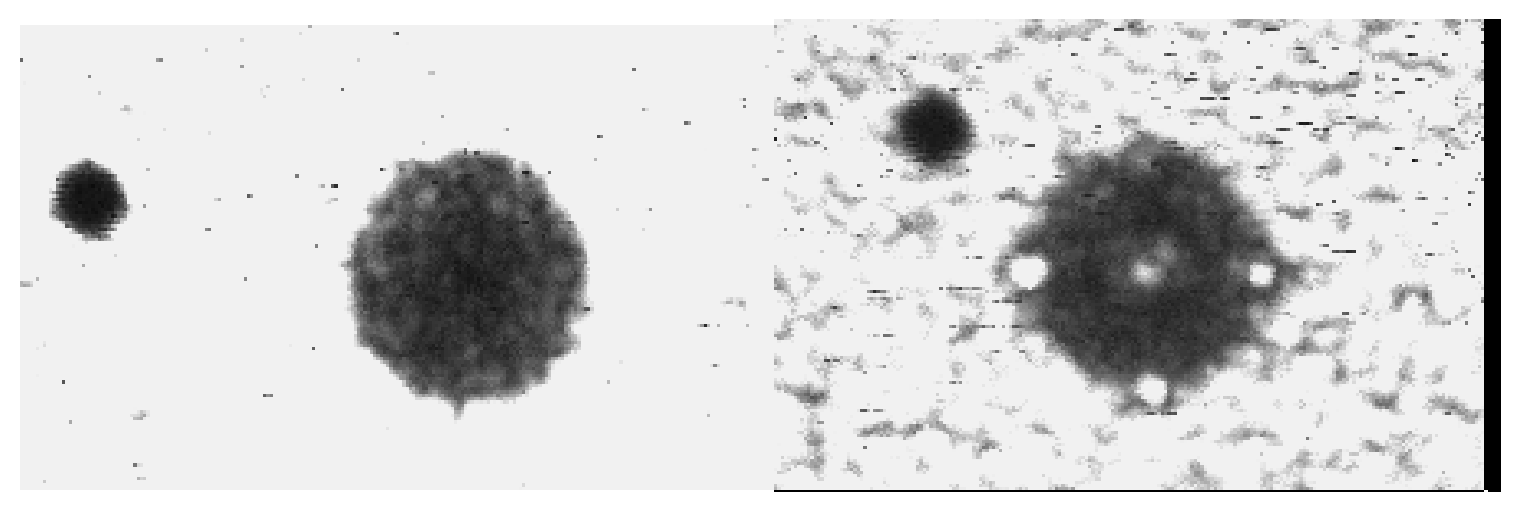

Figure 92: TTU C-scan image of S2-FM94-P7 with (right) and without (left) 5-Hole Repair

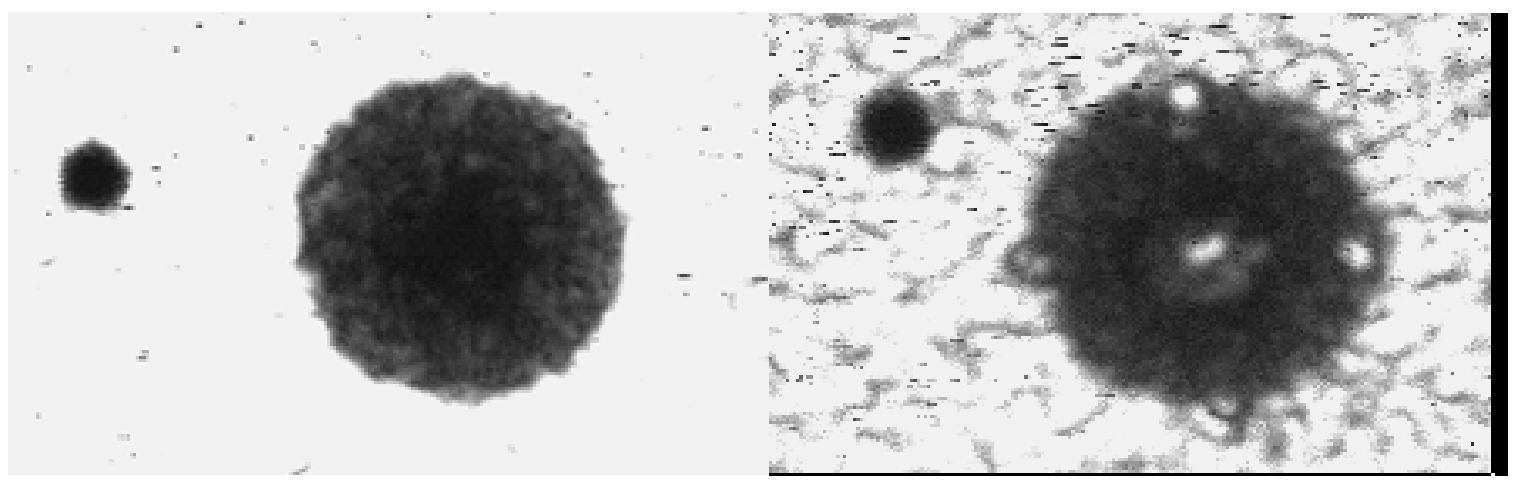

Figure 93: TTU C-scan image of S2-FM94-P13 with (right) and without (left) 5-Hole Repair

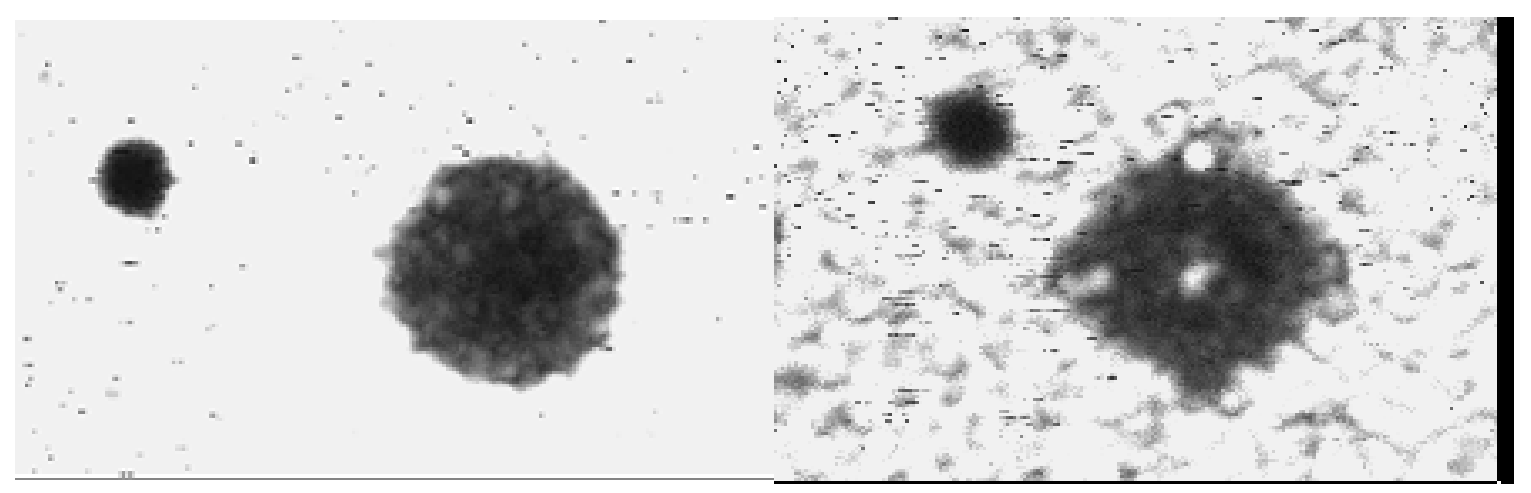

Figure 94: TTU C-scan image of S2-FM94-P9 with (right) and without (left) 5-Hole Repair 

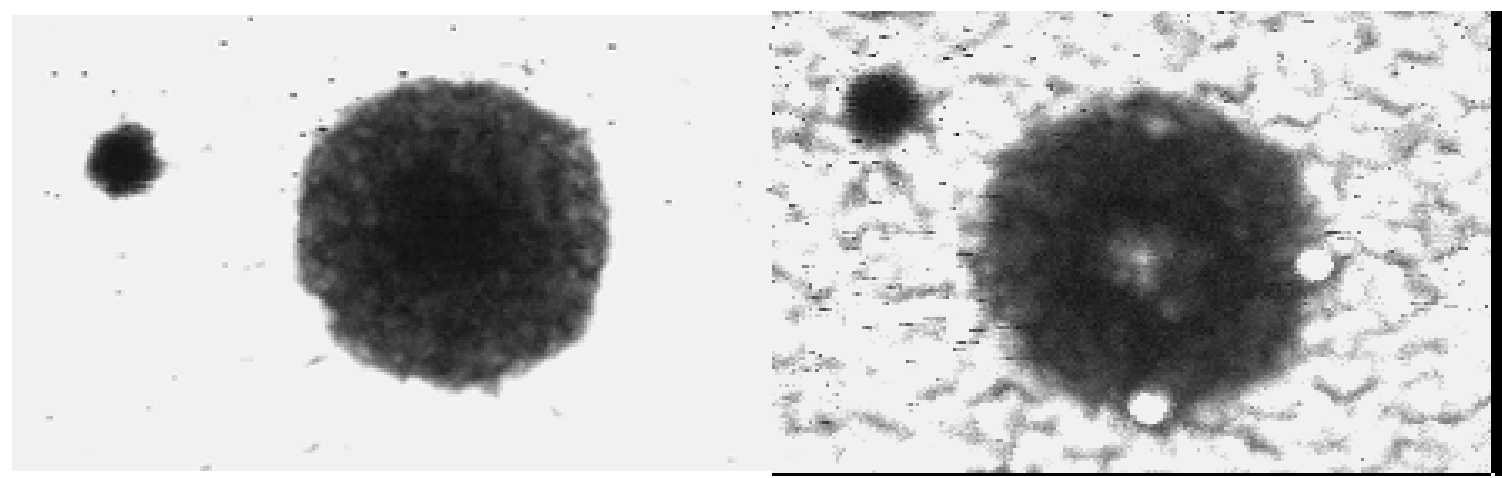

Figure 95: TTU C-scan image of S2-FM94-P14 with (right) and without (left) 5-Hole Repair

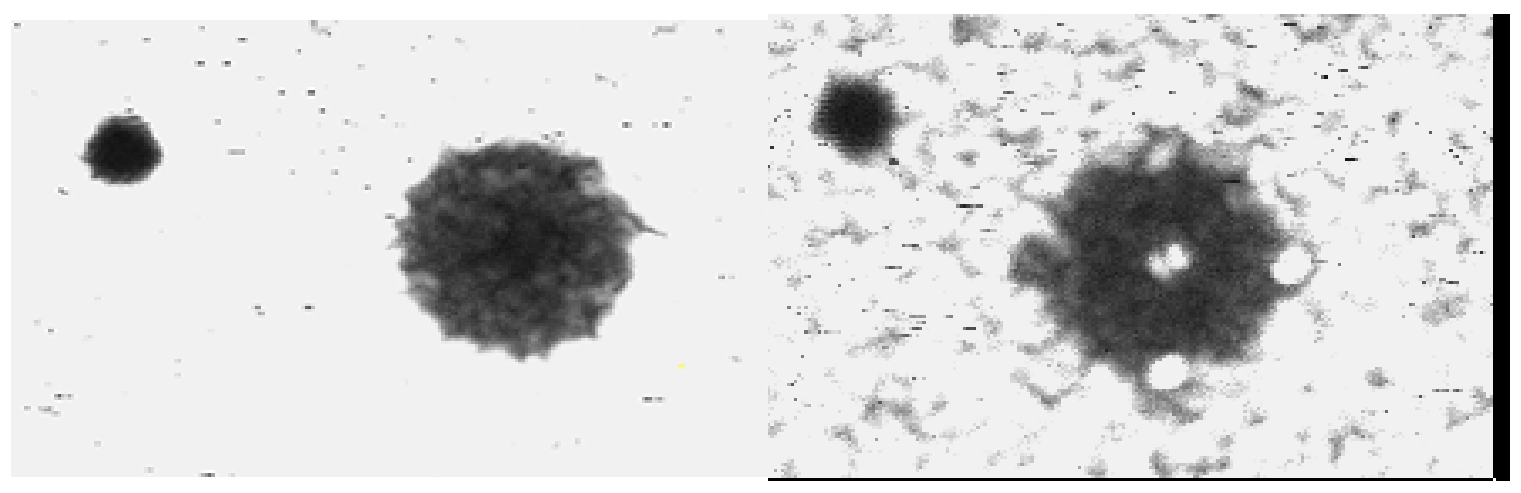

Figure 96: TTU C-scan image of S2-FM94-P17 with (right) and without (left) 5-Hole Repair

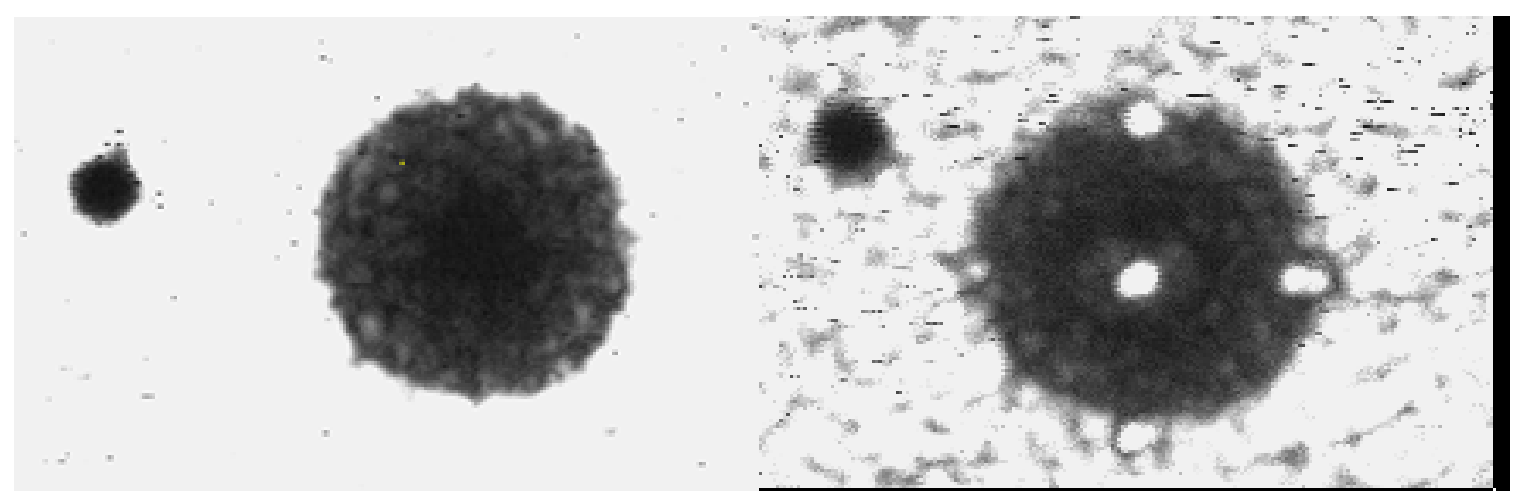

Figure 97: TTU C-scan image of S2-FM94-P19 with (right) and without (left) 5-Hole Repair 
NONDESTRUCTIVE INSPECTIONS OF CRF SANDWICH SPECIMENS WITH AND WITHOUT REPAIR

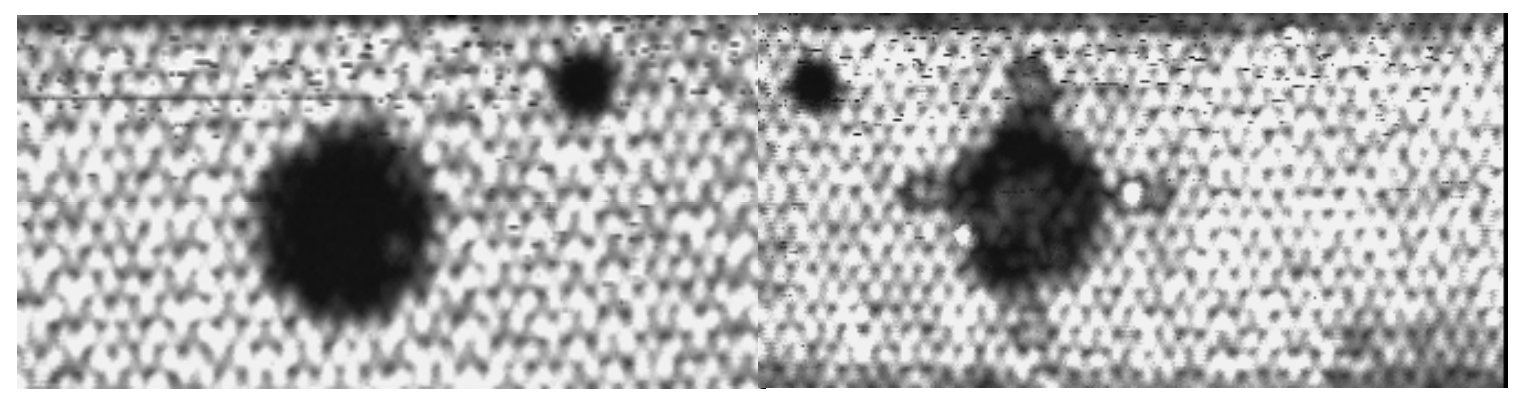

Figure 98: TTU C-scan image of CRF-7 with (right) and without (left) 5-Hole Repair

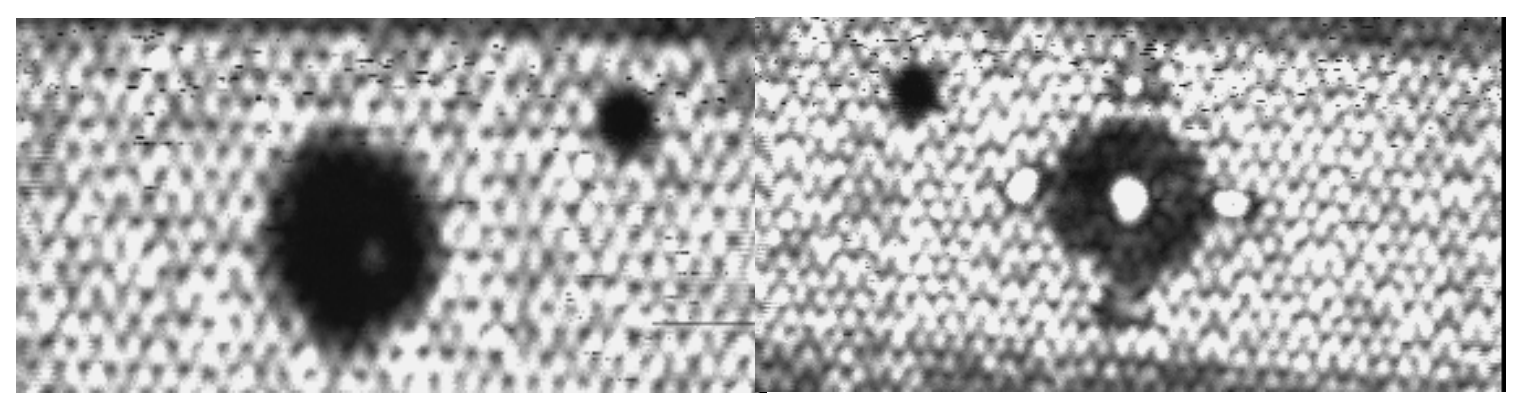

Figure 99: TTU C-scan image of CRF-8 with (right) and without (left) 5-Hole Repair

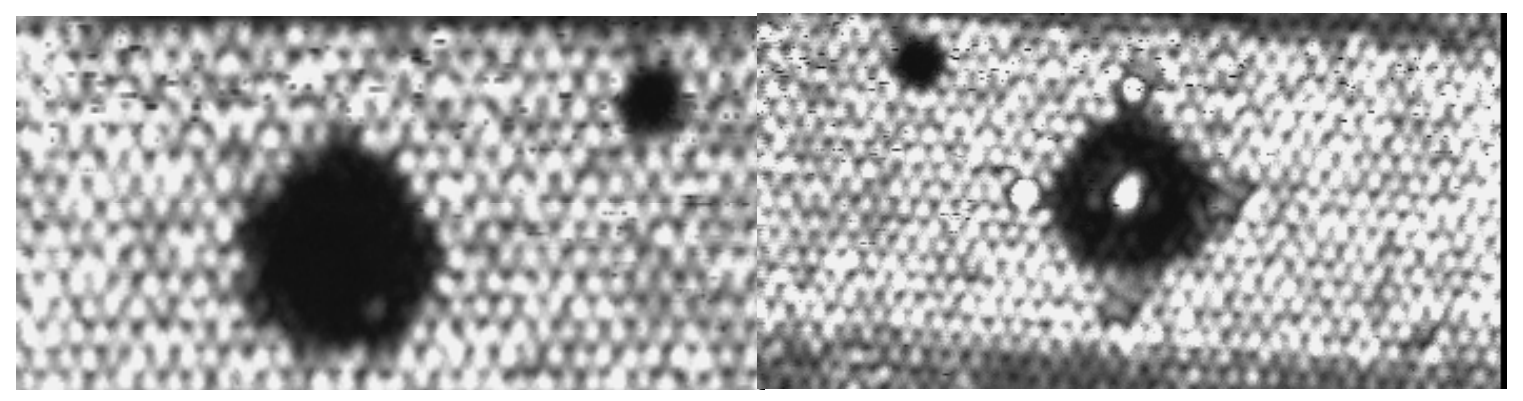

Figure 100: TTU C-scan image of CRF-11 with (right) and without (left) 5-Hole Repair 


\section{APPENDIX F}

NONDESTRUCTIVE INSPECTIONS OF CRF, S2FM94 AND E2-H SANDWICH SPECIMENS WITHOUT FIVE-HOLE REPAIR

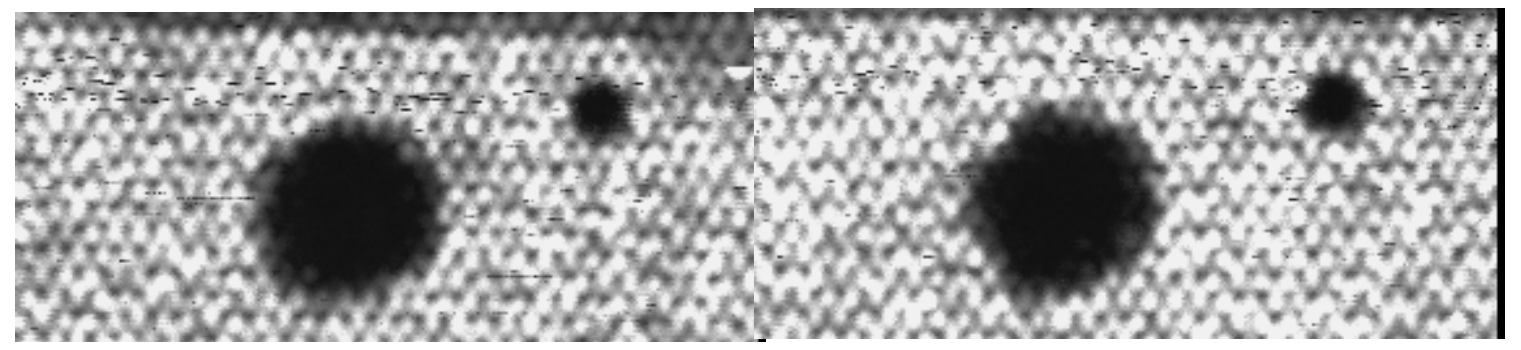

Figure 101: TTU C-scan image of CRF-5 (left) and CRF-6 (right) without 5-Hole Repair
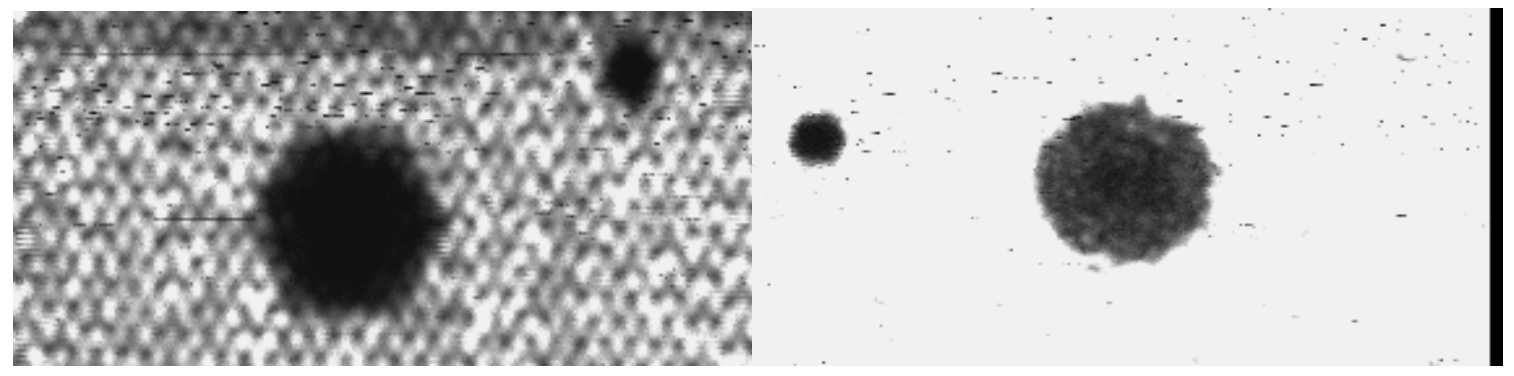

Figure 102: TTU C-scan image of CRF-10 (left) and S2-FM94-16 (right) without 5-Hole Repair

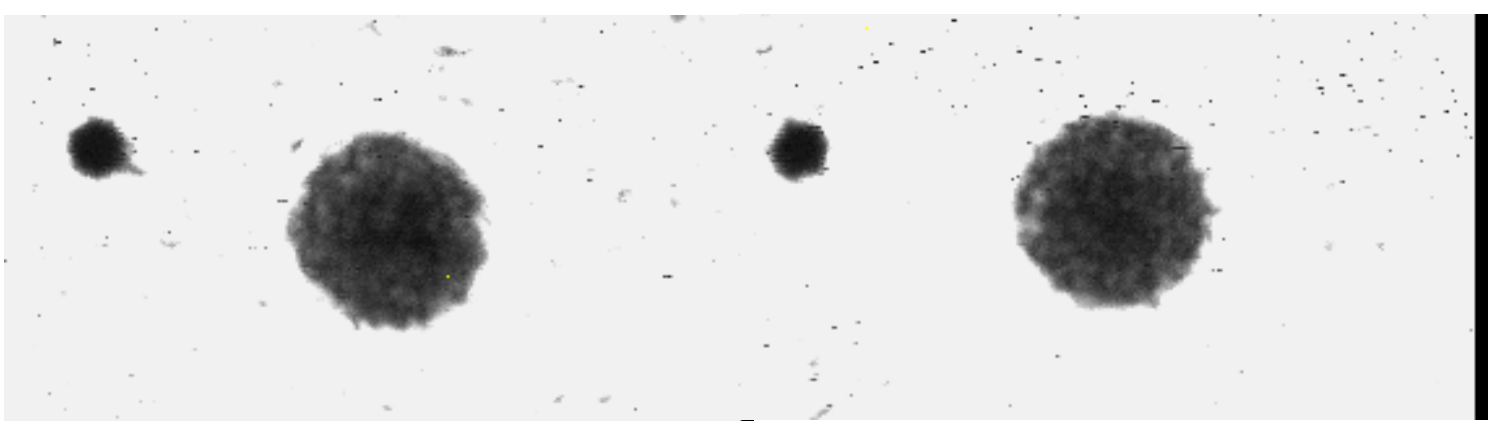

Figure 103: TTU C-scan image of S2-FM94-5 (left) and S2-FM94-6 (right) without 5-Hole Repair 

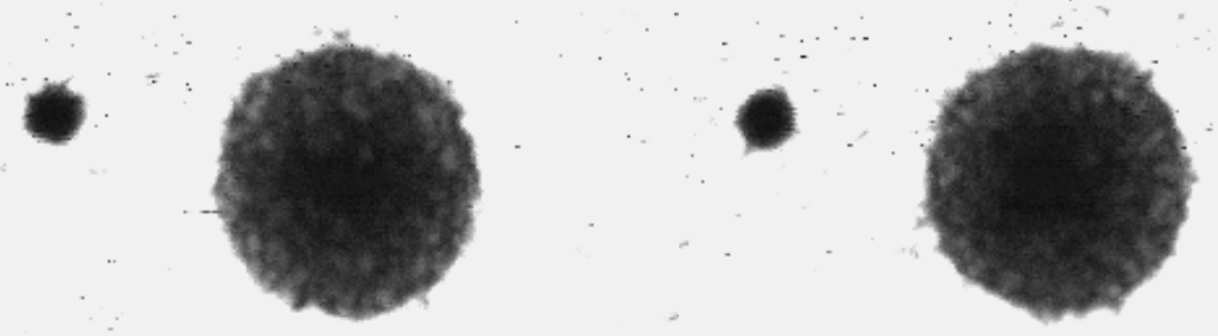

Figure 104: TTU C-scan image of S2-FM94-P11 (left) and S2-FM94-P12 (right) without 5-Hole Repair

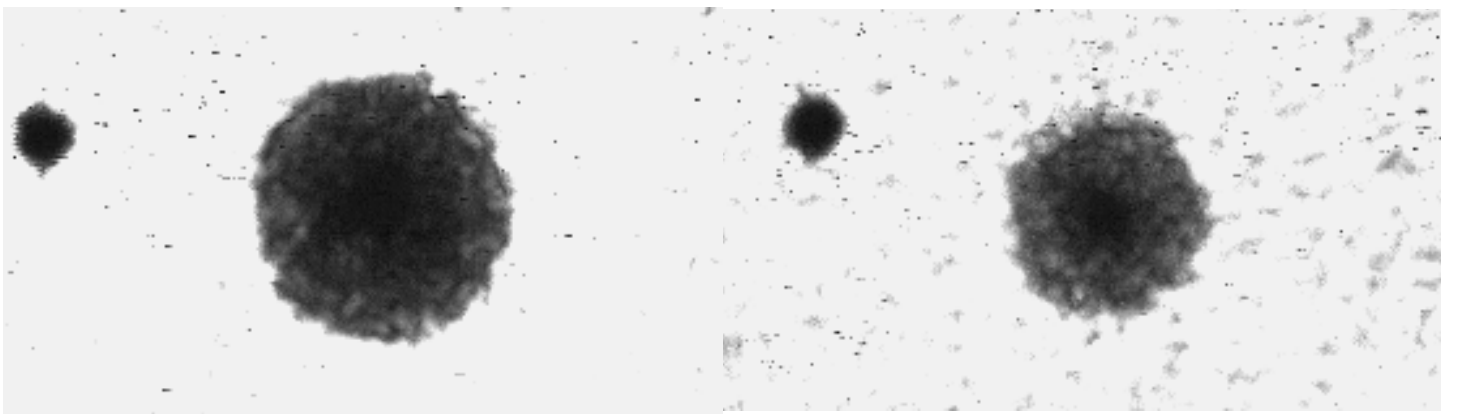

Figure 105: TTU C-scan image of S2-FM94-P18 (left) and E2-H-16 (right) without 5-Hole Repair

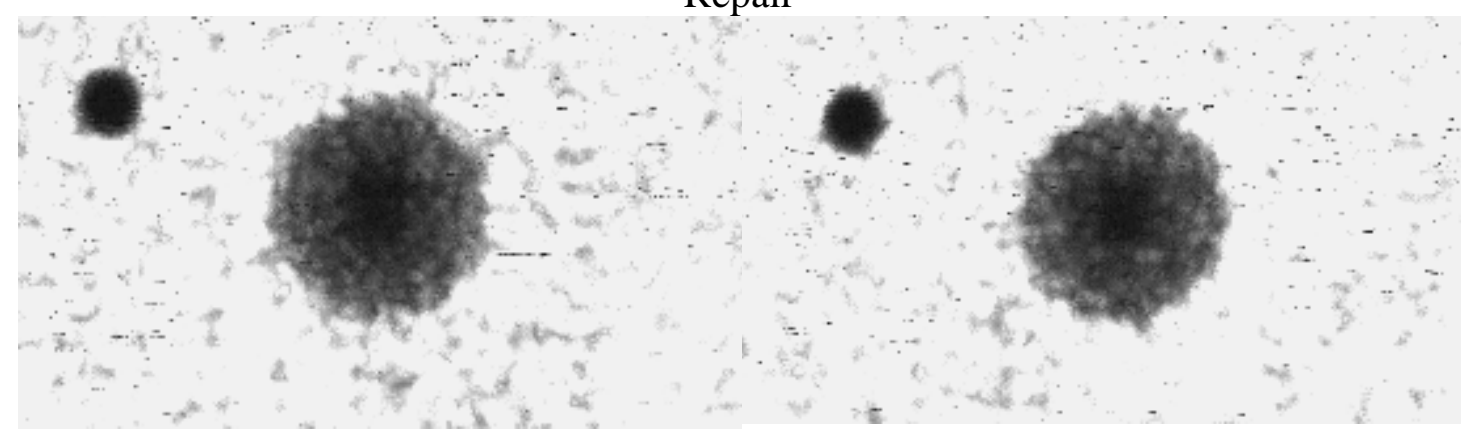

Figure 106: TTU C-scan image of E2-H-5 (left) and E2-H-6 (right) without 5-Hole Repair

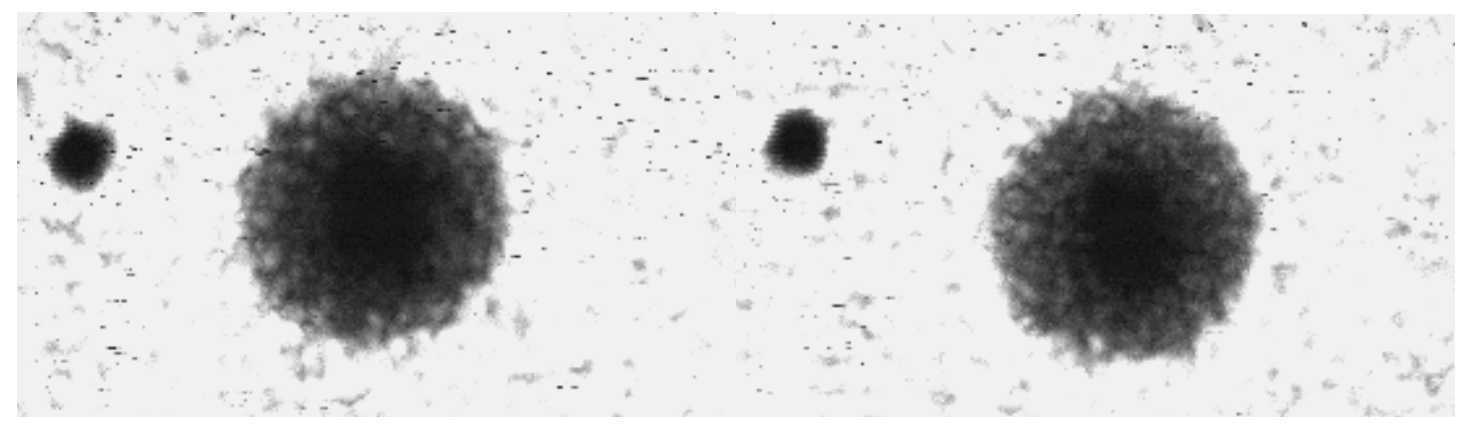

Figure 107: TTU C-scan image of E2-H-10a (left) and E2-H-11 (right) without 5-Hole Repair 


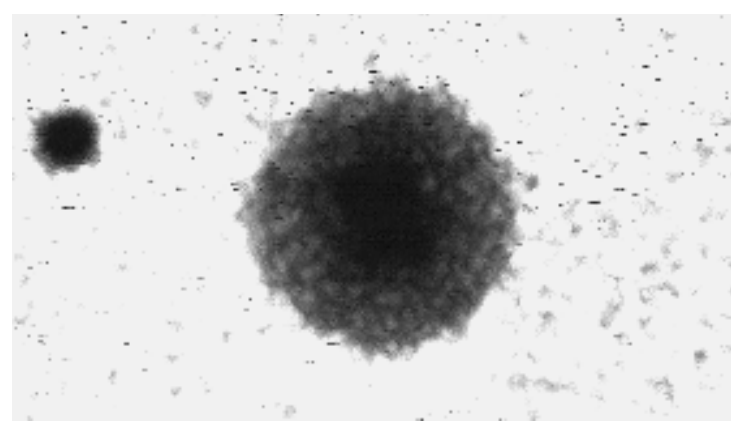

Figure 108: TTU C-scan image of E2-H-18 without 5-Hole Repair 